\title{
The role of Satb2, Ctip2 and Fezl in cortical connectivity and the elucidation of their downstream pathways
}

\author{
Dissertation for the award of the degree \\ "Doctor of Philosophy" \\ Division of Mathematics and Natural Sciences \\ of the Georg-August-University Goettingen
}

submitted by

Paraskevi Sgourdou

From Athens, Greece

Goettingen, 2012 


\begin{abstract}
Prof. Dr. Andreas Wodarz (reviewer)
Department of Anatomy and Cell Biology, Unit of Stem Cell Biology, GZMB
\end{abstract}

Prof. Dr. Ahmed Mansouri (reviewer)

Department of Molecular Cell Biology, Research Group Molecular Cell Differentiation, MPIbpc

Prof. Dr. Ernst A. Wimmer (committee member)

Department of Developmental Biology, GZMB

Date of oral examination: 


\section{Declaration}

This thesis has been written with no other sources and/or aids than those cited.

Paraskevi Sgourdou

February 2012, Goettingen 
To my wonderful mother

"Die Griechen haben den Traum der Menschheit am schönsten geträumt"

Johann Wolfgang von Goethe (1749-1832) 


\section{CONTENTS}

ABBREVIATIONS

ABSTRACT

1.1 Evolutionary aspects of cortical development

1.2 Neurogenesis of the mouse neocortex

1.3 Types of projection neurons within the neocortex

1.4 Commissure formation in the mammalian forebrain

1.5 Special AT-rich sequence Binding protein 2 (Satb2)

1.6 COUP TF-Interacting Protein 2 (Ctip2)

1.7 Forebrain Embryonic Zinc-Finger 2 (Fezf2) protein (or Fezl)

1.7.1 Fezl role in Xenopus and zebrafish

1.7.2 Fezl role in mouse

1.8 Netrin-1 and its receptors

1.8.1 Role of Netrin-1 in axonal outgrowth and guidance

1.8.2 Role of Netrin-1 receptors in neuronal migration, axonal outgrowth and guidance

1.8.2.1 DCC role in axonal projection and neuronal migration

1.8.2.2 Unc5h3 in neuronal migration and axon guidance

1.8.2.3 The emerging role of the DCC family receptor Neogenin

1.8.2.4 The newly identified Down syndrome cell adhesion molecule (DSCAM) 
2.1 Mutant mice $\quad 32$

2.1.1 Satb2 conventional Knockout mice 32

2.1.2 Ctip2 (Bcl11b) conventional Knockout mice 32

2.1.3 Fezf2 (Fezl) conventional Knockout mice 33

2.2 Genotyping $\quad 34$

2.3 Histological procedures $\quad 36$

2.3.1 Tissue processing $\quad 36$

2 3.2 Immunohistochemistry 36

2.3.3 In situ hybridization (Chromogenic and Fluorescent) 37

2.3.4 Nissl Staining $\quad 38$

2.4 DiI tracing $\quad 39$

2.5 In utero injection and electroporation 39

2.6 Molecular biology methods $\quad 40$

2.6.1 Transformation $\quad 40$

$\begin{array}{ll}\text { 2.6.2 Plasmid isolation } & 40\end{array}$

$\begin{array}{ll}\text { 2.6.3 Plasmid linearization } & 40\end{array}$

2.6.4 Purification of linearised plasmid DNA- phenol chloroform $\begin{array}{ll}\text { extraction } & 41\end{array}$

2.6.5 In vitro transcription of linearized plasmid (for cold in situ) 41

2.6.6 Chromatin immunoprecipitation 42

$\begin{array}{ll}\text { 2.6.7 Luciferase assay } & 42\end{array}$

3. RESULTS 44

3.1 Convergence of the Satb2 and the Netrin-1 pathways 44

3.1.1 Altered expression of axon guidance molecules in the $\mathrm{Satb}^{-/-} 44$

3.1.2 Unc5h3 expression pattern is mostly overlapping with Satb2 and mutually exclusive with Ctip2 in the neonatal forebrain 45

3.1.3 Unc5h3 expression is fully restored and callosal projections partially rescued in the $S a t b 2^{-/-} ;$Ctip $^{-/-}$compound mutants $\quad 48$ 
3.1.4 Exogenous introduction of Unc5h3 into the Satb2 deficient neocortex can partially restore the missing callosal projections in a stage specific manner

3.1.5 Upregulation of DCC expression in the cortex of both $\mathrm{Satb2} 2^{-/-}$ and $\operatorname{Satb2} 2^{-/-;} \mathrm{Ctip}^{-/-}$deficient brains

3.1.6 DCC and Unc5h3 are dynamically regulated during embryonic development and their expression, at E14.5, is not affected by Satb2 and/or Ctip2 deletion

3.1.7 Ctip2 directly binds to Unc5h3 promoter elements in vivo, and regulates Unc5h3 expression in vitro

3.2 Mechanisms delineating corticofugal projection identity

3.2.1 Patrial restoration of subcortical projections in the $\mathrm{Satb2}^{-/-} ; \mathrm{Fezl}^{1-}$ compound mutants

3.2.2 Present and grossly normal the corticothalamic projections in all three different mutants $\left(\mathrm{Satb2}^{--}, \mathrm{FezI}^{\gamma_{-}^{-}}\right.$and $\left.\mathrm{Satb2}^{--} ; \mathrm{Fez}^{--}\right)$examined 67

3.2.3 Subcortical projection neuronal identity does not seem to be fully restored in the $\mathrm{Satb2^{-- } ; \mathrm { Fez } ^ { - }}$

4.1 Regulation of callosal projections in the mouse forebrain

4.1.1 Callosal neuron identity downstream of Satb2

4.1.1.1 The control of callosal specification

4.1.1.2 The effect on Unc5h3 expression

4.1.1.3 The effect on DCC expression

4.1.2 Hints for the differential regulation of CPN identity amongst the different cortical layers

4.1.3 The necessity for a dynamic regulation of Netrin-1 receptors' expression throughout development

4.1.3.1 The dynamic expression of DCC and its possible role in axonal guidance 
4.1.3.2 The possible role of Unc5h3 in callosal projections during development and in relation to DCC 82

4.1.4 The possible involvement of additional receptors 84

$\begin{array}{lr}\text { 4.2 Regulation of subcortical projections } & 84\end{array}$

4.2.1 Possible existence of different molecular determinants within distinct subpopulations of DL neurons $\quad 85$

4.2.2 Ectopic subcortical identity of upper layers is regulated independently of Fezl $\quad 86$

$\begin{array}{lr}\text { CONCLUSION } & \mathbf{8 8}\end{array}$

5. APPENDIX $\quad 89$

5.1 Expression of axon guidance molecules and transcription factors in the Satb2 $2^{-/} ;$Ctip2 $^{-/-} \quad 89$

5.2 Ephrinb2 overexpression in wt cortex 91

$\begin{array}{ll}\text { 6. REFERANCES } & 93\end{array}$

$\begin{array}{ll}\text { ACKNOWLEDGEMENTS } & 104\end{array}$

$\begin{array}{ll}\text { CURRICULUM VITAE } & 106\end{array}$ 


\section{ABBREVIATIONS}

$\mathrm{AC}$

$\mathrm{AgCC}$

BG

bp

BSA

$\mathrm{CC}$

cDNA

$\mathrm{CgC}$

CGE

CMV

CNS

COUP-TF

$\mathrm{CP}$

C.P.

CPN

$\mathrm{CPO}$

CR

CST

CSMN

Ctip2

DA

DAPI

DCC

DiA

DiI

DL

DMSO

DNA

dNTP

DSCAM

DTT
Anterior Commissure

Agenesis of the $\mathrm{CC}$

Basal Ganglia

Base pair

Bovine Serum Albumin

Corpus Callosum

complementary DNA

Cingulate Cortex

Caudal Ganglionic Eminence

Cytomegalovirus

Central nervous system

Chicken Ovalbumin Upstream Promoter Transcription Factor

Cortical Plate

Cerebral Peduncle

Callosal Projecting Neurons

solated cleft palate

Cajal-Retzius

Corticospinal tract

Cortico Spinal Motor Neurons

COUP-TF Interacting Protein 2

Dopaminergic

4',6-Diamidino-2-phenylindole

Deleted in Colorectal Cancer

4-(4-dihexadecylamino)styryl)-N- methylpyridinium iodide

1,1'-dioctadecyl-3,3,3'3'-tetramethylindocarbocyanine perchlorate

Deep layer

Dimethylsulfoxide

Deoxyribonucleic acid

Deoxynucleotide triphosphate

Down's Syndrome Cell Adhesion Molecule

Dithiothreitol 
E Embryonic day

EDTA Ethylenediamin-N,N,N',N'-tetra acetic acid

EtOH Ethanol

FAK Focal Adhesion Kinase

Fezf2 (Fezl) Forebrain Embryonic Zinc-Finger 2 (Fezf2)

FGFR1 Fibroblast Growth Factor Receptor 1

FISH luorescent in situ hybridization

Fzd Frizzled

GABA $\quad \gamma$-amino butyric acid

GAPDH Glyceraldehyde-3-phosphate dehydrogenase

GE Ganglionic Eminence

GFP Green Fluorescent Protein

GW Glial Wedge

H.C. Hippocampal Commissure

HCL Hydrochloric acid

HD Homeodomain

HDAC Histone Deacetylase

HEPES 2-[4-(2-Hydroxyethyl)-1-piperazine]-ethanesulfonic acid

HRP Horseradish Peroxidase

IC Internal Capsule

IFL Inner Fibrious Layer

IGG Indusium Griseum Glia

IHC Immunohistochemistry

IPC Intermediate Progenitor Cell

IRES Internal Ribosome Entry Site

ISH In situ hybridization

ISVZ Inner subventricular zone

IZ Intermediate Zone

kDa kiloDalton

LB Luria-Bertani

LGE Lateral Ganglionic Eminence

LV Lateral Ventricle

M1 Primary motor cortex

MARs Matrix attachment regions 


\begin{tabular}{|c|c|}
\hline MGE & Medial Ganglionic Eminence \\
\hline MSN & Medium Spiny Neurons \\
\hline $\mathrm{mM}$ & milliMolar \\
\hline mRNA & messenger RNA \\
\hline $\mathrm{MZ}$ & Marginal Zone \\
\hline MZG & Midline Zipper Glia \\
\hline $\mathrm{NaCl}$ & Sodium chloride \\
\hline NF1B & Nuclear factor-1/B \\
\hline $\mathrm{Ngn} 2$ & Neurogenin 2 \\
\hline $\mathrm{NuRD}$ & Nucleosome Remodelling and Deacetylation \\
\hline $\mathrm{o} / \mathrm{n}$ & overnight \\
\hline OFL & Outer Fibrous Layer \\
\hline OSVZ & Outer subventricular zone \\
\hline PAGE & Polyacrylamide Gel Electrophoresis \\
\hline PBS & Phosphate-Buffered Saline \\
\hline PCNA & Proliferating cell nuclear antigen \\
\hline PCR & Polymerase Chain Reaction \\
\hline PFA & Paraformaldehyde \\
\hline PH3 & Phosphorylated Histone H3 \\
\hline PK & Proteinase $\mathrm{K}$ \\
\hline PKC & Protein Kinase $\mathrm{C}$ \\
\hline PP & Preplate \\
\hline RGCs & Radial Glial Cells \\
\hline RNA & Ribonucleic acid \\
\hline RNase & Ribonuclease \\
\hline rpm & Revolutions per minute \\
\hline RT & Room Temperature \\
\hline RTK & Receptor tyrosine kinase \\
\hline Satb2 & Special AT-rich sequence Binding protein 2 \\
\hline SBD & Smad-binding domain \\
\hline SDS & Sodium Dodecyl Sulfate \\
\hline SEM & Standard error of mean \\
\hline Shh & Sonic hedgehog \\
\hline SNPs & Short Neuronal Precursors \\
\hline
\end{tabular}


SSC Sodium chloride-Sodium citrate

STE Sodium chloride-Tris-EDTA

SVZ Subventricular Zone

TAE Tris-Acetate-EDTA

TAQ Thermophilus aquaticus

TBS Tris-Buffered Saline

TCA Thalamocortical axons

TE Tris-EDTA

Tris Tris (hydroxymethyl)-amino methane

TrK Tyrosine receptor Kinase

TSA Tyramide Signal Amplification

Tween 20 Polyoxyethylene sorbitan monolaurate

UL Upper layer

UTP Uridine Triphosphate

V1 Primary visual cortex

VZ Ventricular Zone

WM White Matter

Wt Wild type 


\section{ABSTRACT}

Satb2 has been shown to be a key postmitotic determinant of callosal projection neurons (Alcamo et al., 2008; Britanova et al., 2008). One way by which Satb2 is acting to confer such identity involves the repression of Ctip2, a transcription factor expressed in corticospinal motor neurons (CSMN) of layer $\mathrm{V}$ and other subcerebral projection neurons within the deep cortical layers (V and VI). In the current study we have found that Unc5h3, a receptor that mediates repulsion to netrin1 signals and is robustly downregulated throughout the whole cortical plate of the $S a t b 2^{-/}$, plays an important role in guiding axons toward the midline. Unc5h3 is mostly expressed in callosal projection (Satb2+) neurons and largely excluded from the subcerebral projection (Ctip2+) neurons of the neonatal mouse cortex. Overexpression of Unc $5 \mathrm{~h} 3$ in the Satb2 deficient neocortex leads to a partial restoration of callosal projections in a stage specific manner (at E12.5 but not E14.5).

Furthermore, the additional deletion of Ctip2 from the Satb2 deficient cortex, accomplished via the generation of Satb2 $2^{-/}$Ctip $2^{-/}$compound mutants, results in the restoration of Unc5h3 expression and in a similar partial rescue of callosal projections. Given that Satb2 deficient neurons show an ectopic upregulation of Ctip2 and a loss of Unc5h3 expression, which is restored upon Ctip2 deletion, we conclude that Ctip2 is repressing Unc5h3. The above conclusion is further supported by the fact that Ctip2 protein can bind to putative binding sites on the Unc5h3 promoter region and repress its expression in vivo and in vitro.

Moreover, the expression of DCC, another netrin-1 receptor that mediates attraction to netrin-1 signals, is upregulated in the superficial cortex of both Satb2 ${ }^{-/}$ and Satb2 $2^{-/}$;tip $2^{-/}$mutants, suggesting that Satb2 is somehow repressing DCC expression. This upregulation can, at least partially, explain the lack of a full callosal restoration in the $\mathrm{Satb2}^{-/}$both upon Unc5h3 overexpression at E12.5 (a time point when deep and superficial layers can be influenced) and after the additional deletion of Ctip2 (i.e. in the Satb2 ${ }^{-/} ; \mathrm{Ctip}^{-/}$).

In parallel, we have studied the effect of the deletion of Satb2 in Fezt $\mathrm{F}^{-/}$mice (Fezl is a transcription factor robustly expressed by CSMN and other subcerebral projection neurons). We have found that unlike $\mathrm{Fezl}^{-/}$mice, that almost completely lack subcortical projections, the $S a t b 2^{-/} ; \mathrm{Fezl}^{-/}$compound mutants have partially 
regained some aspects of their subcerebral connectivity. However, Ctip2 expression does not seem to be fully restored in the deep layers of the compound mutants, while the ectopic Ctip2 expression in callosal UL neurons is maintained similar to what is seen in Satb2 $2^{-/}$mice. The above results imply that the induction of Ctip2 expression by Fezl is most likely not mediated by Satb2; at least not in the cells that express high levels of Ctip2 and project to the spinal cord (type I, layer V neurons). Additionally, the absence of Fezl from progenitor cells (i.e. in the $\mathrm{Satb}^{-\alpha_{-}} ; \mathrm{Fezl}^{-/}$) has clearly no effect on the ectopic expression of Ctip2 in callosal neurons of Satb2 mutants. 


\section{INTRODUCTION}

\subsection{Evolutionary aspects of cortical development}

During the course of evolution the development of the neocortex, which has been present in small mammals since the Triassic period, has changed considerably mainly because of increases in size and complexity. It is known that the enlargement of the mammalian, and even more the human, neocortex occurs primarily via its surface expansion without an analogous increase in its thickness. This is explained by the radial unit hypothesis according to which the symmetrical neural cell divisions before the onset of neurogenesis give rise to radial cortical columns, while later assymetric divisions within each column account for the increase in total cortical surface area (Rakic, P. et al., 1995). The subsequent cortical convolution was facilitated by the presence of radial glial cells that extent fibers which span the entire cerebral wall and, except for neuronal precursors, serve as scaffolds for radially migrating neurons (Gal, J. S. et al., 2006).

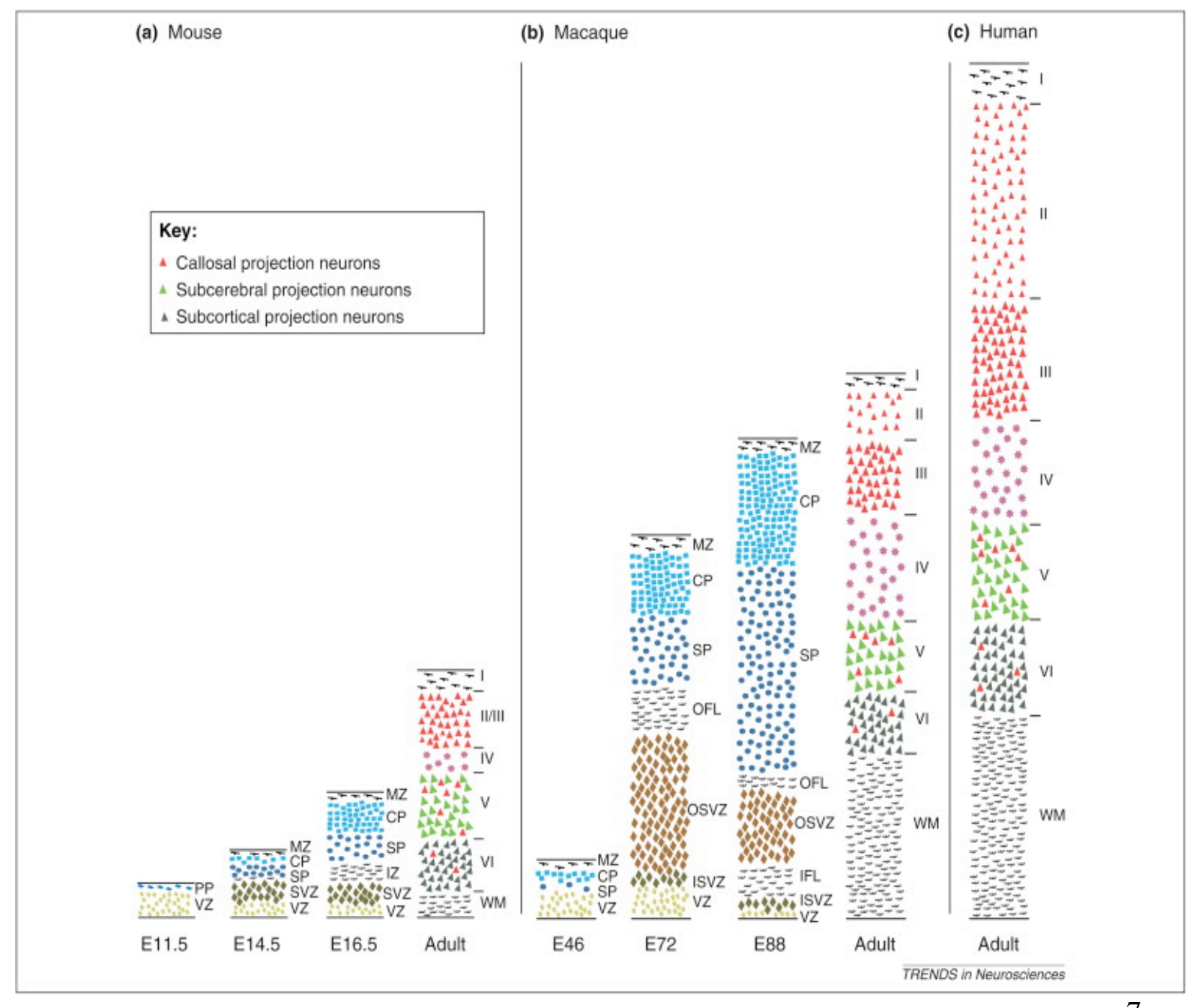


Fig. 1. Comparison of histological sections of mouse, macaque and human embryonic and adult neocortex. Abbreviations: CP, cortical plate; E, embryonic; PP, preplate; VZ, ventricular zone; IFL, inner fibrous layer; ISVZ, inner SVZ; MZ, marginal zone; OFL, outer fibrous layer; OSVZ, outer SVZ; SP, subplate; WM, white matter. The other colors represent: progenitors (yellow/brown), immature neurons (blue), stellate neurons of layer IV (purple), white matter/axons (black). (Adopted from Fame RM. et al., 2011)

During evolution of the cerebral cortex there have been many changes including increase in neuronal number and cell type as well as introduction of new neuronal migratory pathways. For example, the emergence of callosal projection neurons $(\mathrm{CPN})$ took place later in evolution and was first observed in placental mammals. This led to the introduction of a second proliferative zone, except for the ventricular zone (VZ), called the subventricular zone (SVZ) and to the subsequent expansion of neocortical superficial layers. In primates the SVZ is even more expanded and diversified (inner and outer SVZ) giving rise to the bigger and more complex cortex found in primates (Fame, RM et al., 2011). The fact that by mutating even single morphogenetic molecules we can manipulate the cytoarchitectonic specification of the neocortex, can possibly explain how changes might have occurred during evolution.

\subsection{Neurogenesis of the mouse neocortex}

Despite its cytoarchitectural uniformity, the cerebral cortex exhibits a remarkable diversity in terms of both neuronal and glia cell types. During corticogenesis the layer of cells that lines the ventricles is called the ventricular zone (VZ) and comprises of progenitors, which on one hand self renew via symmetrical divisions, and on the other hand give rise to neuronal precursors via assymetrical divisions. Neurogenesis in mice starts around E11.5 and finishes around E17.5 with the sequential generation of postmitotic neurons that position themselves away from the ventricle in a radial fashion. Their migration is facilitated by radial glial cells (basal progenitors), which extend processes all the way up to the pial surface and serve as scaffolds for postmitotic traveling neurons. The first progenitor divisions expand the neuroepithelium and the subsequent assymetric divisions give rise to the preplate, which later on splits into the marginal zone and the underlying subplate. In between these two layers cells migrating radially in such a way that later born neurons 
migrate pass earlier born neurons, a pattern known as the inside out model of corticogenesis, will form the cortical plate (Angevine et al., 1961).

Except for the VZ a second site of progenitor cells, called the subventricular zone (SVZ) and situated right above the VZ, starts to form at arould 13.5. Progenitors of the SVZ, also known as basal progenitors or intermediate progenitor cells (IPCs), give rise to UL neurons as indicated by the expression of similar markers (Svet, Cux2) in both progenitor cells in the SVZ and postmitotic UL neurons (Nieto, M. et al., 2004; Tarabykin, V. et al., 2001; Zimmer, C. et al., 2004). It has been shown that progenitor cells progressively lose their pluripotency (ability to differentiate into any type of cortical cell) since they lose their ability to respond to environmental cues, and thereby follow their intrinsic program (Desai, et al., 2000).

The marginal zone (MZ), or layer I of the neocortex, is populated by CajalRetzius (CR) cells that express Reelin and migrate into the cortex mainly from the cortical hem and the ventral pallium (Bielle et al., 2005). Furthermore, there is a heterogeneity of progenitor types, even within the VZ and SVZ, the differentiation of which seems to depend on both their intrinsic cell program and the extracellular environmental signals (Frantz, G. D. et al., 1996). For example, there is a distinct population of progenitors within the VZ, called short neuronal precursors (SNPs), which differs morphologically and molecularly from the rest of the VZ precursors. This population gives rise directly to postmitotic neurons, whereas the radial glial cells (RGCs) mostly generate neurons indirectly via SVZ cells (Stancik, E. et al., 2010).

\subsection{Types of projection neurons within the neocortex}

As cortical development progresses, neurons of different layers are sequentially born and migrate to populate the appropriate laminar area while progressively acquiring their proper projection identity. One class of neurons found primarily in the UL of the neocortex, and less in layers V and VI, extends axonal projections to the contralateral side through the corpus callosum. These neurons are termed commissural neurons. The second class, includes neurons that project subcortically and can be broadly divided into two subclasses: the corticothalamic neurons, situated in layer VI, that project to the thalamus and the subcerebral projection neurons that project a. to the tectum (corticotectal), b. to the pons 
(corticopontine) and c. to the spinal cord (corticospinal motor neurons, CSMN). All these different types of projection neurons express a variety of different markers that are thought to be, at least partially, responsible for defining neuronal identity. Some widely used such markers can be seen in the figure below.

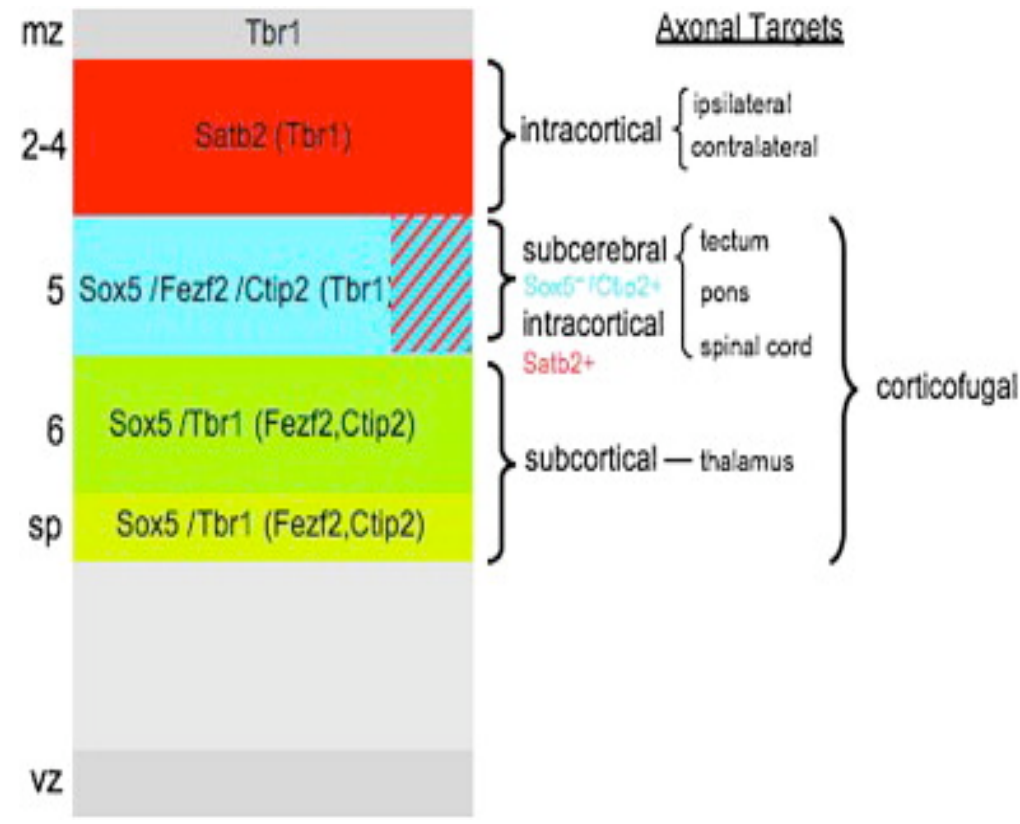

Fig. 2. Scheme showing the expression patterns of some transcription factors that specify different neuronal identities in the neocortex, and thereby ensure appropriate projections. Genes in parentheses are expressed in lower levels. mz, marginal zone; sp, subplate; vz, ventricular zone (adopted from Fishell, G. and Hanashima C., 2008).

\subsection{Commissure formation in the mammalian forebrain}

The proper and more complex connectivity within different parts of the brain is responsible for the higher cognitive functions of the mammalian, and even more of the primate, species acquired during evolution. There are three major commissural tracts formed in the mammalian brain and include a. the corpus callosum (C.C.), b. the anterior commissure (A.C.), and c. the hippocampal commissure (H.C.). One necessary component for commissural formation is the various glial structures located at the telencephalic midline, which make up cellular boundaries surrounding the tracts and regulate the fusion of the brain hemispheres. The latter role is attributed to the midline zipper glia structure while the glial wedge and the indusium griseum, situated ventral and dorsal to the C.C. respectively, are known to secrete various axon guidance molecules necessary for guiding callosal fibers (Shen, W. B., et al., 2006). 
Disruptions of glial structures may have an effect on the development of all axonal commissures. Specifically for the C.C., there are quite a few genes that are known to be expressed in the glial wedge (e.g. FGFR1, NFIB and EphA4) and to be responsible for the proper formation of the above commissure. Additionally, both long-range diffusible factors (as well as various morphogens) and short-range axon guidance molecules, expressed in different areas of the mammalian forebrain, have been shown to play a role in commissure formation. The first axons to cross the midline are those of the A.C. at around E14.5, followed by the hippocampal commissure axons that cross both dorsal and rostral to the A.C. at around E15.5. Lastly, the C.C. fibers are known to have already crossed the midline by E16.5.

The largest, and possibly most

a Commissural; callosal projection neurons

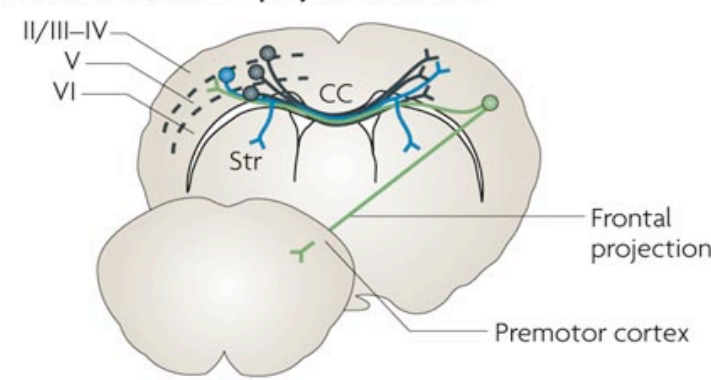

- Callosal neurons (layers II/III, V, VI)

- Callosal neurons with ipsilateral frontal projections (layer V)

- Callosal neurons with striatal projections (layer Va)

b Corticofugal; corticothalamic neurons

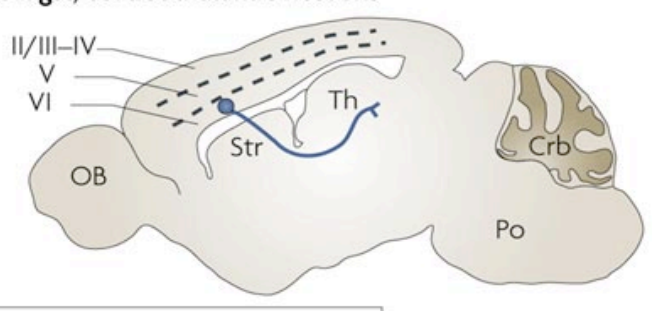

Corticothalamic neurons (layer VI)

c Corticofugal; subcerebral projection neurons

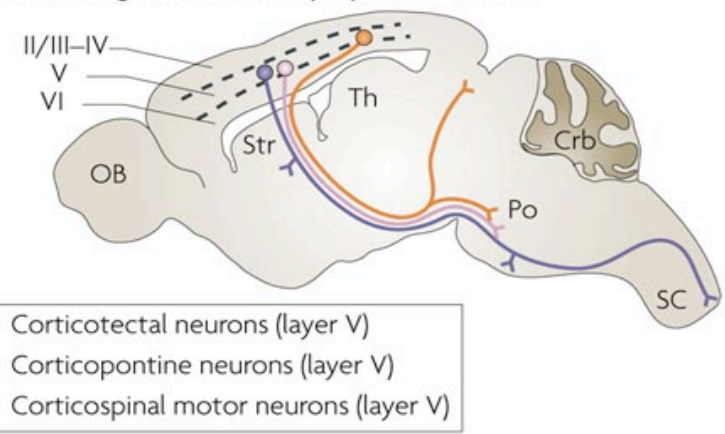

important, structure that connects the two brain hemispheres is the corpus callosum, which in humans consists of more than 200 million axons (Tomasch, J. 1954). The first axons to cross the midline are the ones situated in the cingulate cortex and are called pioneer axons, providing a path for the later arriving callosal axons of the neocortex (Rash, B. G. and Richards, L.J., 2001). In humans, agenesis of the corpus callosum $(\mathrm{AgCC})$ is implicated in many syndromes and neuropsychological disorders with a wide range of causes and an even greater variety of symptoms. In mice there are many gene disruptions that lead to callosal agenesis and can provide, up to a certain extent, a way of modeling human AgCC (Paul, L. K. et al., 2007). 
Fig. 3. Scheme showing the basic categories of projection neurons within the neocortex. Except for the commissural and corticofugal, there are also the associative projection neurons which extent their axons within the same cerebral hemisphere (adapted from Molyneaux et al., 2007).

The development of C.C. in mice has been arbitrarily divided into six sequential steps, seen in Fig. 4 (Richards, LJ. et al., 2004). Each one of these steps is thought to require specific signals in order for the callosal axons to be attracted or repelled from their next or previous intermediate target respectively (see figure legend). Although the callosal pioneering axons of the cingulate cortex are known to cross the midline at the same developmental stage throughout the whole rostrocaudal axis of the C.C., the caudal callosal neurons of the neocortex cross the midline approximately one day later compared to rostral. It is hypothesized that even though the rostral callosal fibers of the neocortex require the cingulate pioneering axons to guide them, the caudal callosal axons are using the hippocampal commissure for the same purpose.

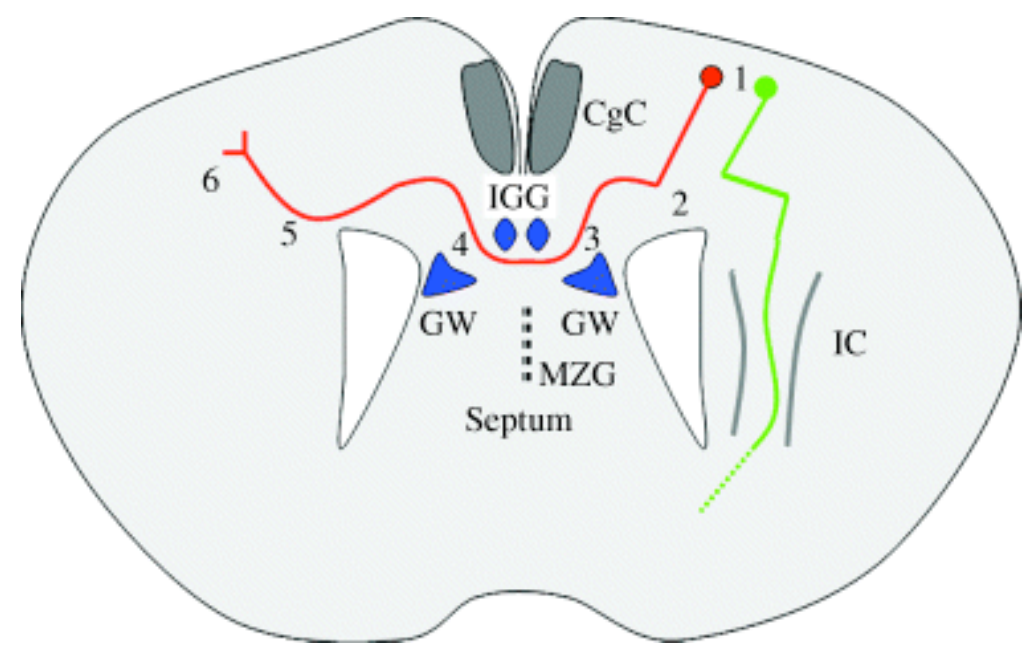

Fig. 4. Sequential steps in callosal neuron projection. Callosal neurons 1 . project ventrally toward the intermediate zone, 2. turn medially, and not laterally, to project through the cingulate cortex in a steep ventral trajectory, 3. make an abrupt turn in the cortico-septal boundary and cross the midline, 4. turn dorsally into the contralateral cingulate cortex and subsequently into the neocortex where they grow until 5. they find their target area where they use radial glial fibers to grow dorsally and 6 . innervate their final target.

IC, internal capsule; $\mathrm{CgC}$, cingulate cortex; IGG, indusium griseum glia; GW, glial wedge; MZG, midline zipper glia. (Adapted from Richards, LJ. et al., 2004). 


\subsection{Special AT-rich sequence Binding protein 2 (Satb2)}

Satb2 was first identified as the second member, after Satb1, of the family of nuclear matrix-attachment region (MARs) binding proteins implicated in transcriptional control and chromatin remodeling. The first report by FitzPatrick et al. (2003) showed that haploinsufficiency of Satb2, caused by a translocation that interrupts its transcription, can lead to isolated cleft palate (CPO). Several cases of microdeletions in the 2q33.1 region, which includes Satb2, have been reported to result in similar clinical features (mental and growth retardation, dysmorphic features, feeding difficulties and cleft or high palate) (Rosenfeld, J A. et al., 2009; Urquhart, J. et al., 2009). Congenital syndromes, like Toriello-carey syndrome, characterized by C.C. agenesis, cleft palate, mental retardation and facial dysmorphology have been atributed to interruption of the Satb2 gene (Tegay, D.H. et al., 2009).

Satb2 protein shows a remarkable sequence similarity between humans and mice with only three amino acid substitutions. Similar to Satb1, Satb2 was shown to bind to MAR sequences and associate with the nuclear matrix. Furthermore, Satb2 can be sumoylated in lysine residues 233 and 350, resulting in the reduction of its transcriptional activation potential and its localization to the nuclear periphery (Dobreva et al., 2003). In the mouse nervous system, Satb2 mRNA is detected at E13.5 in the telencephalon and in a subpopulation of dorsal spinal cord neurons. At E14.5 Satb2 positive cells begin their migration towards the cortical plate and by E15.5 many of them have already reached the CP, where almost half of the neurons express Satb2. By P0 most Satb2 positive cells occupy the upper part of the cortical plate, with some expression in the DL as well, whereas in the adult brain expression is maintained in the neocortex and part of the hippocampus although at much lower levels (Britanova et al., 2005).

The fact that Satb2 is part of a nuclear complex interacting with nuclear MARs has also been shown in vivo, in tissue isolated from E18.5 cerebral cortices. Similar to mice, Satb2 protein has an analogous expression pattern in the developing neocortex of rats, with high expression levels in a subset of postmitotic differentiating neurons that are born before E17 (Szemes, M. et al., 2006). Except for the radially migrating Satb2+ postmitotic neurons, there is also a smaller population of projection 
neurons born in the SVZ that migrate tangentially in the upper part of the IZ to finally populate the subiculum (Britanova et al., 2006).

Deletion of Satb2 leads to various craniofacial abnormalities with defects in jaw and palate development, due to haploinsufficiency. The Satb2 $2^{-/}$mice exhibit callosal agenesis with no formation of Probst bundles, while the hippocampal commissure seems normal and the anterior commissure is much thicker. The absence of Satb2 from the neocortex leads to the ectopic upregulation of Ctip2 in the Satb2 deficient neurons, changing their UL genetic program to that of DL. This has a direct effect on the projection of Cre expressing (Satb2-) neurons which instead of projecting callosaly they are misguided into either forming subcortical projections or transversing the anterior commissure (Fig. 5).

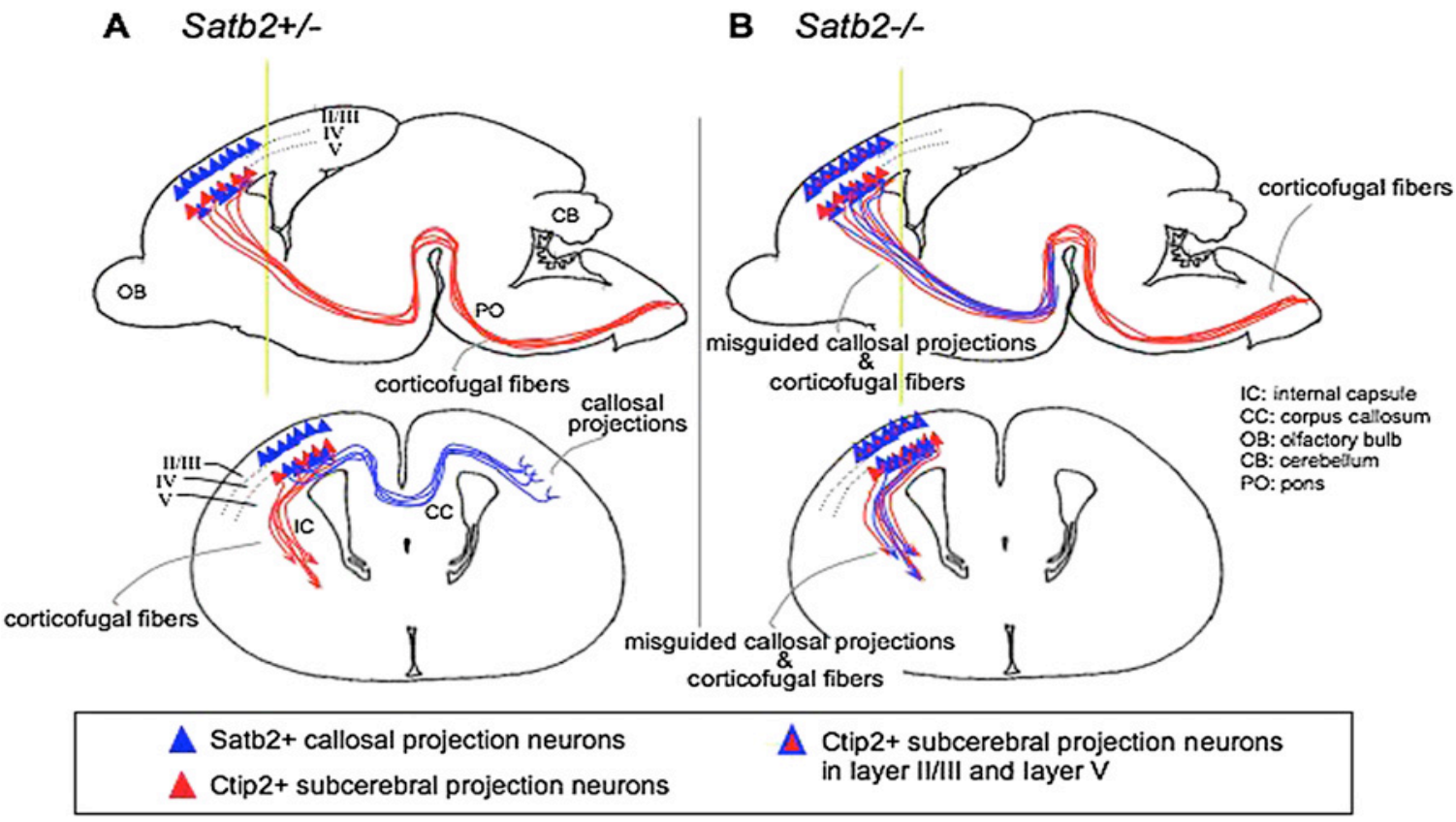

Fig. 5. Scheme depicting the abnormal projections observed in $S a t b 2^{-/-}$mice. Sagittal and coronal sections of $\mathrm{Satb2}^{+/}$(A) and $\mathrm{Satb2}^{-/-}$(B) mice. The ectopic expression of Ctip2 (red) in callosal projection neurons (blue) leads to the misrouting of these neurons toward subcortical projections through the internal capsule (IC). The redirected axons travel along with corticofugal fibers all the way to roughly the level of substantia nigra (adopted from Fishell, G. and Hanashima C., 2008).

Furthermore, Satb2 has been shown to interact with the nucleosomeremodeling $(\mathrm{NuRD})$ complex and to directly bind to the Ctip2 promoter thereby repressing its expression (Britanova et al., 2008; Alcamo et al., 2008). In the absence of Satb2, the AU-rich element RNA binding protein 1 (AUF1) has been shown to 
replace Satb2 in the chromatin-remodeling complex in rat cortical neurons (Gyorgy, A. et al., 2008). The conserved Satb2 sequence and expression patterns across different vertebrate species (human, mouse, chick, zebrafish) are indicative of the functional conservation of Satb2 during evolution (Sheehan-Rooney K. et al., 2010).

\subsection{COUP TF-Interacting Protein 2 (Ctip2)}

The highly related Ctip1 and Ctip2 zinc finger proteins (88\% identity) were first isolated in a yeast two-hybrid screen, where they were shown to interact with all members of the Chicken Ovalbumin Upstream Promoter Transcription Factor (COUP-TF) Orphan Nuclear Receptors (Avram, D. et al., 2000). However, Ctip1 was later shown to be a sequence specific DNA binding protein that is capable of functioning independently of COUP-TF family members (Avram, D. et al., 2002). Similarly, Ctip2 was also shown to interact with histone $\mathrm{H} 3 / \mathrm{H} 4$ deacetylases (like SIRT1) and to mediate transcriptional repression in mammalian cells by binding to GC-rich response elements in a COUP-TF independent manner (Senawong, T. et al., 2003). Furthermore, Ctip2 was found to associate with the NuRD complex and to co-

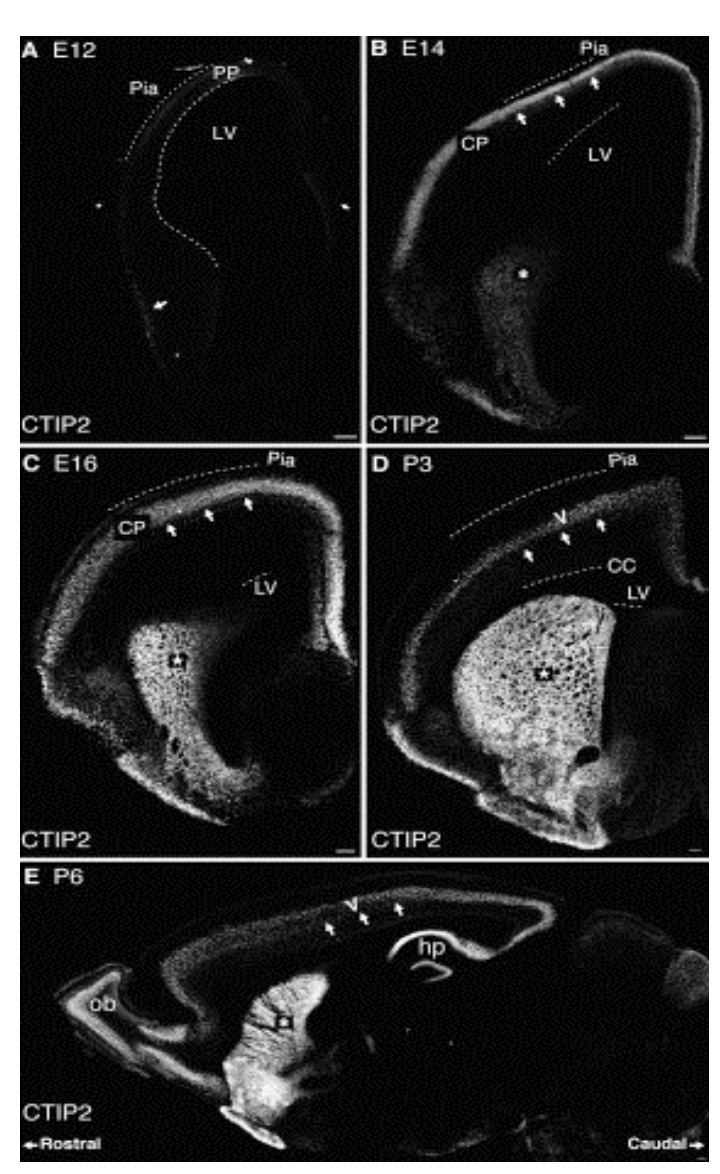
occupy the promoter template of p57KIP2 gene (a cyclin dependent kinase inhibitor) whereas at the HIV-1 promoter region it has been shown to recruit HDAC1 and 2 . When bound to DNA, it associates with methyltransfereases to increase local histone methylation (Marban, C. et al., 2007; Topark-Ngarm, A. et al., 2006).

The human homologues of Ctip1 and Ctip2 are the B-cell leukemia 11A and B (Bcll1a and $b)$ which, except for the fetal brain, are highly expressed in germinal center B-cells and were directly implicated with immune system malignancies (Satterwhite, E. et al, 2001). Since $B c l 11 b$ was expressed preferentially 
Fig. 6. Expression of Ctip2 in embryonic and postnatal mouse brain. At E12 Ctip2 expression is restricted to a small part of the lateral cortex (A) while at E14 its expression has become robust in the developing CP and the striatum (B). By E16 the signal can be detected in the CP neurons destined to become deep layer neurons (C) and at early postnatal stages the expression is confined to neurons of layer V and the striatum (D). In a sagittal section of a P6 brain it can be seen that Ctip2 is expressed in layer $\mathrm{V}$ throughout the whole rostal to caudal axis of the cortex, in the striatum, the hippocampus and the olfactory bulb (E). CP: cortical plate, LV: lateral ventricle, hip: hippocampus, ob: olfactory bulb, asterisks indicate the striatum (Arlotta et al., 2005).

in malignant T-cell lines derived from patients with adult T-cell leukemia/lymphoma, knock out mice were generated for studying thymocyte differentiation (Wakabayashi, et al., 2003). The expression analysis of both Ctip1 and Ctip2 in the mouse CNS during embryogenesis and into adulthood revealed partially overlapping, but not identical, expression patterns (especially in the cortex and hippocampus) arguing for possible non-redundant roles during CNS development (Leid et al., 2004).

The isolation and expression profiling of corticospinal motor neurons (CSMN) led to the identification of Ctip2 as one of the transcription factors expressed exclusively by CSMN and other closely related subcerebral projection neurons of layer V and VI (Arlotta et al., 2005). Early in neurogenesis, at E12.5, Ctip2 is expressed in a small cluster of cells in the ventromedial cortex, whereas by E14.5 the expression is very robust throughout the developing $\mathrm{CP}$ and in the striatum. At E16.5, when CSMN and other subcerebral projection neurons have already settled in the CP and have started extending their axons, Ctip2 expression is high in these cells and completely absent from the VZ and/or the SVZ. This indicates that Ctip2 is a postmitotic determinant of CSMN and other subcerebral projection neurons of layer $\mathrm{V}$ and VI and that it might be involved in the proper differentiation (axonal outgrowth/pathfinding) of these neurons. Indeed, Ctip2 null mice exhibit a disorganized striatum with defasciculation of axonal projections through the I.C., while the much fewer CSMN axons that manage to enter the pons never reach the spinal cord. The above extension and fasciculation problems are also observed in the Ctip2 heterozygous animals indicating a gene dosage effect.

The expression of Ctip2 in the striatum is very robust since it is expressed by the striatal medium spiny neurons (MSN), which comprise 95\% of striatal neurons (Arlotta, et al., 2008). In the absence of Ctip2 the MSN, altough they are specified 
from progenitors and migrate into the mantle zone, do not differentiate properly and therefore the striatal patches display an abnormal development.
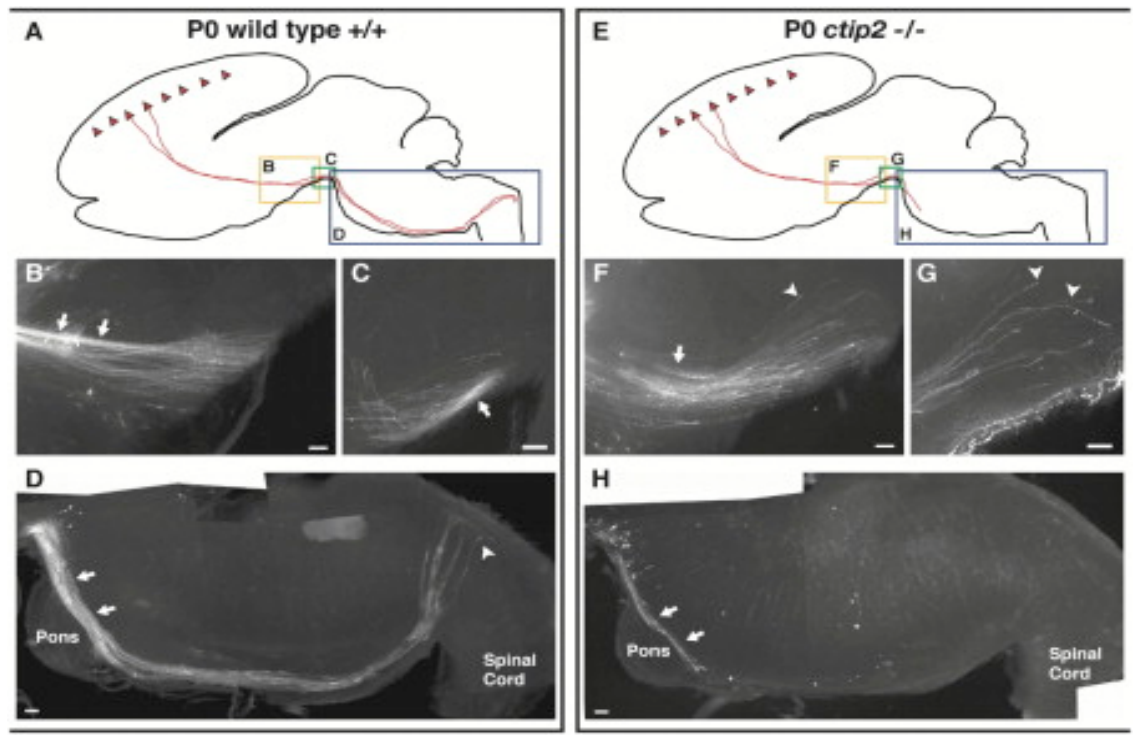

Fig. 7. Projection defects of CSMN neurons in Ctip2 $2^{-/}$mice. Pictures B, C, D and F, G, H are photomicrographs of the boxed areas depicted in the schematic representation of sagittal sections of wild type (A) and $\mathrm{Ctip}^{-/-}$(E) brains respectively. In the Ctip2 mutants there are much fewer CSMN axons reaching the pons $(\mathrm{H})$ compared to wild type mice (D), and their axonal fascicles appear disorganized (F) while many axons extend to ectopic targets (G). The CSMN axons in the Ctip2 $2^{-/-}$ never reach the pyramidal decussation $(H)$ (Arlotta et al., 2005).

\subsection{Forebrain Embryonic Zinc-Finger 2 (Fezf2) protein (or Fezl)}

\subsubsection{Fezl role in Xenopus and zebrafish}

The Fez gene was first identified and cloned as a novel transcription factor containing six $\mathrm{C}_{2} \mathrm{H}_{2}$ type zinc finger domains highly conserved among Drosophila, zebrafish, mouse and human (Matsuo-Takasaki, M., et al., 2000). It has been shown that Fez mRNA is expressed during mid to late-gastrulation at the rostro-medial regions of the anterior neural plate in Xenopus embryos and localizes within the prospective telencephalic region while neurulation progresses. A subsequent study in zebrafish identified a similar to fez protein $(95,7 \%$ identity within the zinc finger regions), which was named fez-like (Fezl). 
The fezl transcript was detected in the anterior edge of the neuroectoderm, the prospective dorsal forebrain, as well as the ventral forebrain overlying the prechordal plate from the late gastrula to mid-segmentation stage during zebrafish development (Hashimoto, H. et al., 2000). Interestingly, Fezl expression was elevated in embryos where Wnt signaling was inhibited and reduced in the ones expressing Wnt8b, indicating a role in the induction of anterior neuroectoderm. This role was further reinforced by the fact that overexpression of fez in zebrafish embryos induces ectopic expression of $d l x 2$ and $d l x 6$, two genes involved in brain development, whereas its morpholino-based knockdown inhibits $d l x 2$ expression in the ventral forebrain (Yang, Z. et al., 2001). Additionally, Fezl gain of function embryos exhibit a selective downregulation of Wnt1 and Pax2a while its misexpression leads to the expansion of the telencephalon and hypothalamus at the expense of other forebrain and midbrain regions (Jeong, J-Y. et al., 2007).

Furthermore, the zebrafish mutant too few (tof) in which the fezl gene is disrupted displays deficits in the development of diencephalic monoaminergic neurons in a non-cell autonomous way (Levkowitz, G. et al., 2002). This is most likely accomplished by inducing the expression of neogenin 1 with which tof is coexpressed in DA progenitor domains of the basal forebrain (Jeong, J-Y. et al., 2006). Fezl control on hypothalamic differentiation is also exerted by its regulation of the homeodomain protein Orthopedia (Opt) at two distinct hypothalamic nuclei that produce isotocin expressing (IT) and DA neurons (Blechman, J. et al., 2007). Except for embryonic development in zebrafish, fezl is also expressed by radial glial progenitor cells of the adult telencephalic ventricular zone as well as by postmitotic neurons during adult neurogenesis (Berberoglu, M.A. et al., 2009).

\subsubsection{Fezl role in mouse}

In mouse embryos the fezl transcript can be detected at E11 in both rostral telencephalic hemispheres with high expression in the cortex and hippocampus and fainter expression in the anterior hypothalamus and the preoptic area (MatsuoTakasaki, M., et al., 2000). The function of Fezl in mouse was revealed after the generation of Fezl mutant mice (Hirata, T. et al., 2004). This study showed that Fezl expression is apparent in the mouse prospective forebrain as early as E8.5. At E13.5 Fezl is detected in the nasal septum, thalamic eminence, ventral thalamus and 
hypothalamus and dorsal telencephalon while by E15.5 it can be found in the cortical plate, striatum, hypothalamus and mamillary body. As development progresses its expression in the cortex is restricted to deep layers, mostly subcerebral projection neurons of layer $\mathrm{V}$ and to a much lesser extent in layer VI (Molyneaux, BJ. et al., 2005). In the Fezl mutants, Ctip2 expression in the neocortex is lost while in the

A

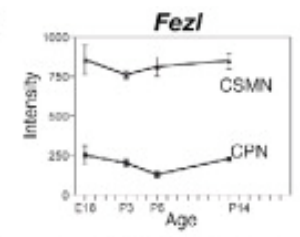

B

C E13.5 Fezl

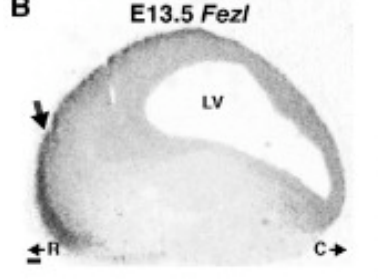

D

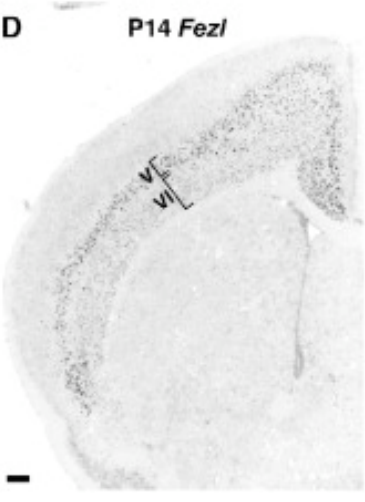

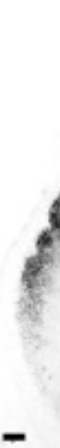

E

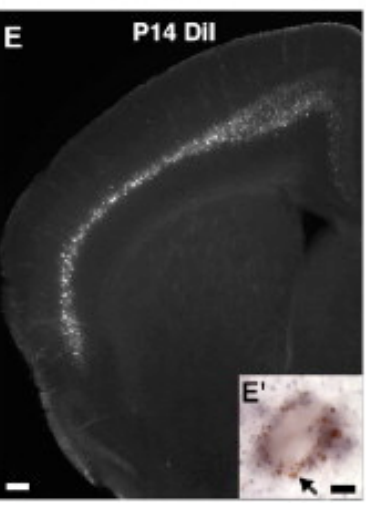
piriform cortex and the striatum it remains unaltered. Furthermore, there is a complete loss of subcerebral projection neurons of layer $\mathrm{V}$ whereas layer VI is expanded and its neurons fail to mature. The neuronal projections to the spinal cord, normally formed by CSMN neurons, are completely absent from the motor cortex (Chen, B. et al., 2005) and the subplate neurons do not develop normally resulting in the subsequent reduction and abnormal development of thalamocortical axons (TCA) (Komuta, Y. et al., 2007).

Fig. 8. Expression pattern of Fezl mRNA at different developmental stages. A. Enrichment of Fezl in CSMN neurons. B and C. Fezl expression in the cortex at E13.5 shows a high rostrolateral- low caudomedial pattern. D. As development progresses expression gets restricted mainly to layer $\mathrm{V}$ and less to layer VI neurons. E. Retrograde labeling with DiI from the pons shows staining in layer V (Molyneaux, BJ. et al., 2005).

Moreover, overexpression of Fezl has been shown to induce expression of Ctip2 in neurons that do not normally express this transcription factor. On the other hand, even though upon Fezl overexpression the CST can be rescued and the UL neurons can acquire altered axon targeting, this is done without any effect on Satb2 expression and with no induction of Ctip2 (Chen, B., 2008). The above show that Fezl regulates a fate switch between subcortical and callosal projection neurons and that it 
does so, both by inducing the expression of Ctip2 as well as through a Ctip2 independent pathway.

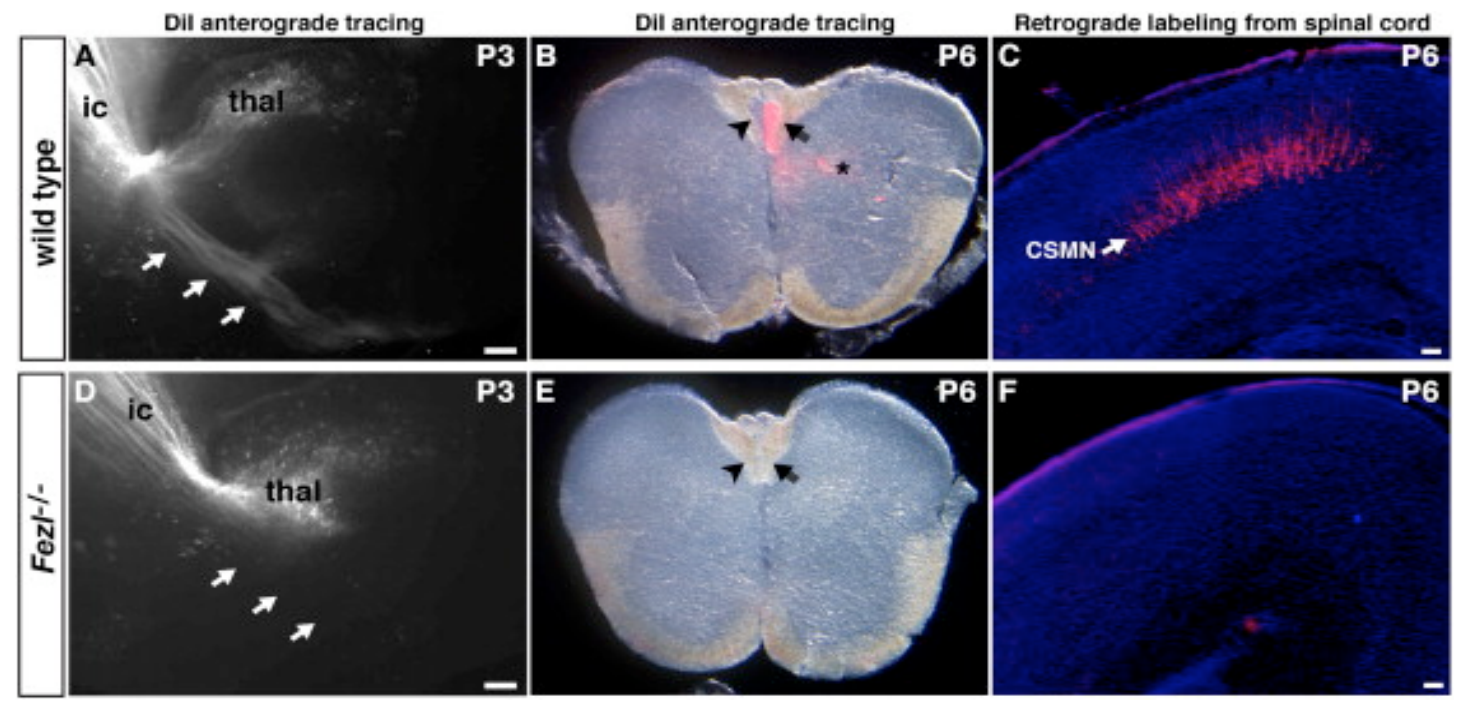

Fig. 9. Absence of subcerebral projection neurons in the Fez $\Gamma^{-}$mice. A., D., B. and E depict anterograde labeling in wt and Fezl mutants showing the presence of corticothalamic and the absence of subcerebral projections (D) in the $\mathrm{Fez}^{-\alpha}$ that never reach the spinal cord (E). C and F. Retrograde labeling from the S.C. shows the absence of layer $\mathrm{V}$ neuronal labeling in the mutant cortex (Molyneaux, BJ. et al., 2005).

Both Fez and Fezl are expressed in the developing forebrain in overlapping and non-overlapping patterns. During early forebrain development Fez and Fezl have been shown to control the differentiation of progenitor cells by repressing the transcription factor Hes5 thereby alleviating its repression on neurogenin2 (Shimizu, et al., 2010). Recently the transcription factor Tbr1 (expressed primarily in layer VI corticothalamic projection neurons) was shown to directly bind Fezl and repress its expression. In Tbr1 deficient mice there is an ectopic expression of Fezl in Tbr1- cells and a misprojection of these neurons to form CST. Conversely, ectopic expression of Tbr1 in layer V neurons abolishes Fezl expression and CS tract formation, whereas overepxression of Tbr1 in Fezl deficient layer V neurons switches their identity into becoming corticothalamic neurons (Han, W. et al., 2011; McKenna, W.L. et al., 2011).

In the Fezl mutant mice, a recent report has shown a reduced number of layer $\mathrm{V}$ and an increased number of UL interneurons. This phenotype was accompanied by restricted neuronal activity in the DL since these neurons, which now acquire a partial 
callosal identity, do not recruit the interneurons appropriate for DL where there is normally a greater inhibition of neuronal activity (Lodato, et al., 2011).

\subsection{Netrin-1 and its receptors}

\subsubsection{Role of Netrin-1 in axonal outgrowth and guidance}

The first description for the in vivo role of netrin-1 in mice came from the study of Serafini et al. (1996) where it was shown that mice homozygous for a hypomorphic netrin-1 allele had severe defects in spinal commissural axon trajectories of the ventral spinal cord. In the brains of mutant animals both the corpus callosum and the hippocampal commissure are absent, the fimbria malformed and the anterior commissure severely defective. A further elucidation of the role of netrin-1 in axonal outgrowth and directionality in the murine telencephalon was provided by the studies of Metin et al. (1997) and Richards et al. (1997).

In the first study, the authors provide evidence that explants of the lateral ganglionic eminence (LGE) and the sulcus between the lateral and medial ridges of the GE (ISS), isolated from E12.5 and E13.5 mouse telencephalon, can attract the axons growing out of cortical explants placed at a distance in a three dimensional matrix. The above effect was at least in part attributed to netrin-1, which is normally expressed by GE subdivisions of the mouse ventral telencephalon. This conclusion was reinforced by the observation that soluble recombinant netrin-1 protein elicits a similar effect to that seen with LGE and ISS explants, which can be suppressed by the application of a function blocking anti-netrin antiserum. In the second study, the authors showed that coculture of internal capsule explants, isolated from E15 rat embryos, with cortical explants leads to the axonal growth of the latter toward the internal capsule. Given that, in vivo, during the first stages of cortical axogenesis the axons extend predominately toward the internal capsule, it was postulated that the above structure secretes a soluble signal that attracts cortical axons. One of the molecules robustly expressed by the internal capsule throughout development is netrin-1. Additional coculture experiments of cortical explants with either E13 rat floor plate, which naturally expresses netrin-1, or with cells transfected with a netrin1 expressing construct showed a similar increase in axonal outgrowth toward the source of netrin-1. 
Additionally, except for its role in attracting corticofugal axons, netrin-1 has been implicated in the proper guidance of hippocamposeptal and septohippocampal projections (Pascual et al., 2004) as well as in the establishment of appropriate thalamocortical connections (Braisted et al., 2000; Powell et al., 2008). The necessity for the chemorepulsive role of netrin-1 has also been shown for various other cell types like the trochlear motor neurons and the oligodendrocyte precursor cells (Colamarino et al., 1995; Jarjour et al., 2003).

Both netrin-1 and floor plate have been implicated in the proper migration of inferior olivary (IO) neurons which show severe defects in their location and their projections to the cerebellum in netrin-1 deficient mice (Bloch-Gallego, E. et al., 1999). Midline netrin-1 expression is also needed for the appropriate migration of progenitor cells from the lower rhombic lip to the ventral midline in order to form the pontine nuclei (Alcantara et al., 2000). Except for embryonic development, the role of netrin-1 in migration has also been shown for the mature nervous system where it is implicated in the chemorepulsive effect on adult nervous stem cells that migrate to the site of injury (Petit et al., 2007).

The molecular elucidation of the bifunctional role of netrin-1 began with the study of Hong et al., 1999. In this study the authors showed that netrin-1 triggers the formation of a receptor complex between DCC and Unc5 receptors, which results in the conversion of the DCC mediated attraction to DCC/Unc5 mediated repulsion. The receptors' association takes place via their cytoplasmic domains, which are important for mediating the repulsive response. Upon ligand (netrin-1) binding the repression of the interaction between the cytoplasmic domains of DCC and Unc5 is relieved, thereby promoting their association and the subsequent downstream transduction of the repulsive signal.

\subsubsection{Role of Netrin-1 receptors in neuronal migration, axonal outgrowth and guidance}

\subsubsection{DCC role in axonal projection and neuronal migration}

Studies on axon guidance mutants in $C$. elegans gave the first indications that $u n c-5$ and unc-40 genes (the homologues of Unc5h3 and DCC respectively) could 
encode for Unc-6 (Netrin-1) receptors (Hedgecock EM et al., 1990). The cloning of these genes and their potential to act as receptors, based on structural characteristics, was later confirmed with in vitro biochemical experiments which showed that both molecules can bind recombinant Netrin-1 (Keino-Masu, K. et al., 1996; Leonardo, ED. et al., 1997). Inactivation of the DCC gene in mice led to severe defects in the projections of spinal commissural neurons, similar to those observed in Netrin-1 deficient mice. Additionally, major commissures in the $D C C^{+-}$brains were absent (corpus callosum and hippocampal commissure) while others (anterior commissure) appeared severely defective (Fazeli, A. et al., 1997). Except for the forebrain commissures, several defects were also observed in midbrain structures in DCC deficient mice. The mesencephalic dopaminergic (DA) neurons that have been shown to express high levels of DCC exist in reduced numbers in midbrain nuclei of $\mathrm{DCC}^{/-}$ mice, while the migration of DA precursors is impaired. Furthermore, the DA ventral commissure and the innervation of the ventral striatum appeared abnormal. At the same time, a reduced number of DA neurons sent projections to the cerebral cortex (Xu, B. et al., 2010). DCC is also expressed by locus coeruleus neurons the migration of which is impaired in $D C C^{-/}$but not $D C C^{k a n g a}$ or $U n c 5 h 3^{-/}$mice (Shi, M. et al., 2008).

The expression pattern of DCC protein follows the axonal growth phase during cortical development with high lateral expression at E13, when axons of the subplate and layers V and VI make their way to the internal capsule, which is later downregulated in these axons but maintained, at E16, in medially projecting axons (Shu, T. et al., 2000). DCC protein expression is also very robust in the developing hippocampus, starting from E13 and continuing throughout the whole phase of hippocampal axonal growth. DCC can be detected in all hippocampal regions (dentate gyrus and CA1-CA3) and the hippocampal commissure, as well as in the anterior commissure during the time of axonal extension and navigation to their long ranged targets. In the septum, the expression of DCC protein is restricted to the ventromedial aspects of the septal VZ.

The mRNA detection of DCC is possible earlier in development, at E11.5, in postmitotic neurons of the newly forming preplate and is downregulated later in development, further supporting the notion of DCC expression in growing neurons (Gad, M.J. et al., 1997). Interestingly, at E13.5 DCC expression was shown to be more intense in the outer and inner edge of the preplate with much lower levels in the 
centrally placed neurons. Even at E9.5 a punctuate expression of DCC mRNA could be detected in the outer most layer of the developing hindbrain. In the ventral telencephalon DCC is robustly expressed in both the thalamus and hypothalamus, but its expression in the former is entirely lost by E15.5 while it is still present in the hypothalamus by E18.5. The hippocampal expression of DCC mRNA was maintained strong throughout the whole period of gestation (Gad, M.J. et al., 1997).

The response of DCC expressing neurons to netrin-1 is increased upon translocation of DCC to the plasma membrane. It has been shown that DCC associates with a G-protein coupled receptor, the membrane associated adenosine $\mathrm{A} 2 \mathrm{~b}$, which is actually a receptor for netrin- 1 that induces accumulation of cAMP upon binding of netrin-1 (Corset, V. et al., 2000). In embryonic spinal commissural neurons a great percentage of DCC protein is localized in intracellular pools, and upon netrin-1 application there is an increase in cell surface DCC. This increase, which requires PKA (Protein Kinase A) and adenylate cyclase activation and is depended on exocytosis, results in axon extension. However, in embryonic rat spinal commissural neurons the netrin-1-induced increase in cell surface DCC takes place via a PKA-independent mechanism (Bouchard, J-F. et al., 2004). A subsequent study from the same group showed that PKA activation is essential for DCC recruitment and translocation in embryonic cortical neurons via a mechanism that requires exocytosis. This recruitment of DCC to the plasma membrane from a preexisting vesicular pool is the result of an increase in the intracellular concentration of cAMP. Additionally, it was demonstrated that depolarization activates PKA, PI3 kinase and PKC thereby increasing the amount of cell surface DCC and promoting cortical axon outgrowth (Bouchard, J-F. et al., 2008).

Furthermore, a study from a different group showed that netrin-1 is inhibiting RhoA, which is, in turn, reducing the amount of plasma membrane DCC. In this way, the binding of netrin-1 is recruiting additional DCC to the plasma membrane via a positive feedback loop through RhoA inhibition (Moore, SW. et al., 2008). In cortical primary neurons the exocytosis dependent DCC changes were observed in the axon shafts but not in the growth cones (Matsumoto, H. et al., 2010). An interesting recent study implicated the translational machinery with a DCC mediated translation in neuronal axons and dendrites. Specifically, it was shown that DCC interacts both physically and functionally, via its cytoplasmic domain, with the translation initiation machinery in spinal commissural axon growth cones and colocalizes with newly 
synthesized protein (Tcherkezlan, J. et al, 2010). DCC has also been shown to interact with Myo X, an unconventional myosin, implicated in cell adhesion and filopodia elongation. The expression of Myo X leads to the distribution of DCC in the tips of cultured neurons and its silencing, in vivo, results in impaired axon projection (Zhu, X-J. et al., 2007).

DCC has been shown to interact directly, via its intracellular domain, with ERK-1 and induce ERK-dependent transcriptional activation (Elk-1 and SRE regulated gene expression). MEK-1/2 is also interacting with DCC and it may be bound to it, but not activated, even before the netrin-1-induced activation and recruitment of ERK1/2. In DCC and netrin-1 deficient animals it is also suggested that commissural axons display a DCC-dependent activation of ERK in response to netrin-1 (Forcet, C. et al., 2002). Moreover, netrin-1 induced cortical neurite outgrowth has been shown to take place through the phosphorylation of PLC $\gamma$ (phospholipase $\mathrm{C} \gamma$ ) in a DCC-P3 depended manner (Xie, Y. et al., 2006). The vicinity of the P3 domain is also the target of the netrin-1 induced phosphorylation of DCC by Fyn tyrosine kinase. This leads to the activation of a guanine nucleotide exchange factor (GEF) that in turn activates Rac1, resulting in actin assembly at the plasma membrane and axonal outgrowth (Meriane, M. et al., 2004). One guanine exchange factor that has been implicated in the netrin-1 induced attractive signaling is DOCK180. More specifically, DOCK180 has been shown to form a protein complex with DCC to which it can bind directly, thereby inducing axon outgrowth and attraction to netrin-1 in primary neurons as well as in commissural axon projections of chick embryos (Li, X. et al., 2008).

Interestingly, the environmental factor heparan sulfate (HS) has been shown to play a cell autonomous role in DCC-dependent neuronal outgrowth and signal transduction in response to netrin-1. In other words the role of HS, as a coreceptor for the netrin-1 morphogen, which has been established both in vivo and in vitro might compliment its existing role as an extracellular stabilizer of diffusible molecules (Matsumoto, Y. et al., 2007). A recent study, implicated the tripartite motif protein related to MID-1, MADD-2, in the Unc-40 mediated axon attraction and branching in C. elegans. This study, gives the first direct evidence for the long inferred notion that individual axonal branches are selectively guided (Hao, JC. et al., 2010). 


\subsubsection{Unc5h3 in neuronal migration and axon guidance}

The rostral cerebellar malformation $(\mathrm{rcm})$ gene was first identified as a spontaneous recessive mutation on mouse chromosome 3 (Lane, P. W. et al., 1992) that resulted in duplication of an exon and an Rcm protein with 55 additional amino acids. The above mutation caused a defective lamination in lateral regions of the rostral cerebellar cortex. A second transgenic insertion allele was later identified $\left(\mathrm{rcm}^{\text {tg }}\right)$ that similar to the previously described spontaneous mutation $\left(\mathrm{rcm}^{\mathrm{s}}\right)$, resulted in various cerebellar and midbrain defects in mice homozygous for the mutant allele (Ackerman, SL. et al., 1997). These defects include a dramatic reduction in cerebellar size, already by P0, defects in laminar structure of cerebella and abnormal Purkinje and external granule cell migration (Ackerman, SL. et al., 1997 and Goldowitz, D. et al., 2000). This abnormal rostral migration of the above cell precursors, results in their accumulation in ectopic areas and the subsequent displacement of the rostral external germinal layer. Additionally, the above phenotype combined with the sites of Unc5h3 and netrin-1 expression, is suggestive of the possible role of Unc5h $3 /$ netrin- 1 repulsive signaling in the establishment of the rostral cerebellar boundary (Przyborski, SA. et al., 1998). More specifically, netrin-1 mRNA is detected in the median sulcus and basal plate of the fourth ventricle whereas the expression of Unc5h3 is observed in cerebellar primordia located to the caudal/dorsal and lateral aspects of the fourth ventricle, juxtaposed to the netrin-1 expression.

A third mutant allele, produced by a transgenic insertion that resulted in the deletion of intron 1 of the $U n c 5 h 3$ gene was also identified. These mice, that had no Unc5h3 expression in most tissues, showed a phenotype similar to the other two previously described as well as ataxia, hyperactivity and reduced fat pads in various tissues (Choi, YS. et al., 2003). The role of Unc5c in cell migration has also been established for the spinal accessory motor neurons (SACMN) the cell bodies of which fail to migrate away from the floor plate midline, along a dorsal pathway towards the lateral exit point, in Unc5c mutants (Dillon, AK. et al, 2007). Similar defects involving proper SACMN migration have also been reported for mice lacking Netrin1 or DCC (Dillon, AK, et al., 2005).

The onset of Unc5h3 expression during mouse neurogenesis is at E8.5 in the neural folds of the neuroectoderm. By E9.5 and E10.5 Unc5h3 can be detected in the 
dorsal mesencephalon, dorsal diencephalon, cerebellar plate and in the neuroepithelium of the lateral regions of the fourth ventricle. At E11.5 its expression is primarily detectable in the cerebellar plate and the dorsal mesencephalon and diencephalon, while at E12.5 there is a robust expression in the differentiating zone, ventricular zone and rhombic lip of the cerebellar primordium. In the spinal cord, the expression is high in motor columns and much lower in the dorsal regions (Przyborski, SA. et al., 1998). In an E14.5 embryo, high expression can be detected in various cerebellar regions as well as in certain hypothalamic nuclei of the ventral telencephalon (our data). During postnatal stages and into adulthood Unc5h3 is expressed in the lateral olfactory tract, in pyramidal cells of the hippocampus, in pontine nuclei and posterior mammillary nuclei. Except for the CNS, Unc5h3 expression is also found in many other tissues throughout embryonic development.

The mouse $r c m$ gene product, as well as the two rat $U n c 5 h 1$ and $U n c 5 h 2$ gene products, was shown to be homologous to the C. elegans Unc-5 gene and to bind soluble netrin-1 protein in vitro (Leonardo, ED. et al., 1997). Except for Unc5h3, the mouse homologues of Unc5h1 and Unc5h2 as well as the newly identified family member Unc5h4, were later cloned (Engelkamp, D. et al., 2002). Two independed reports have specified the subdomains of DCC and Unc5h3 to which netrin binds. More specifically, it has been shown that soluble Netrin (sNetrin) binds to and requires both immunoglobulin repeats of Unc5h3 and the fifth (and/or fourth) fibronectin type III repeat of DCC, which is both necessary and sufficient for binding (Geisbrecht, BV. et al., 2003; Kruger, RP. et al., 2004). The second Ig domain of Unc5 has been shown to have an autoinhibitory effect in tyrosine phosphorylation, whereas the binding of netrin to its receptors is known to take place via multiple domains. Additioanally, netrin-1 stimulation of Unc5 tyrosine phosphorylation requires the activity of Src and FAK kinases, both of which can directly phosphorylate Unc5 in vitro ( $\mathrm{Li}, \mathrm{W}$. et al., 2006). The above Unc5 phosphorylation is dependent on the P3 domain of DCC, reinforcing the notion of the Unc5/DCC receptor complex needed to mediate a repulsive response. Furthermore, another member of the Unc5h family, Unc5ha, is shown to activate RhoA and to a lesser extent Rac1 and Cdc42 during neurite extension in vitro (Picard, M. et al., 2009).

The role of Unc5h3 in neuronal migration was demonstrated by the analysis of the cerebellar defects observed in the Unc5h3 mutants (Ackerman, SL. et al., 1997). Further analysis of the mutant phenotype however, led to the identification of a role of 
Unc5h3 in axonal guidance as well. Although the trajectories appeared normal above the level of the pyramidal decussation, homozygous $U n c 5 h 3^{r c m}$ mice displayed defects in the CST at the level of the dorsal funiculus (Finger, JH. et al., 2002). Similar to $U n c 5 h 3^{r c m}$, mice homozygous for a spontaneous mutation in the DCC gene, DCC ${ }^{\text {kanga }}$, showed abnormal decussation of all CST axons whereas Unc5h3 mutation affected only the midline crossing of some. In gene dosage experiments, Unc5h3 and DCC were shown to act synergistically in guiding part of CST axons. Additionally, defects in the pyramidal decussation and the dorsal funiculus were also observed in Ntn1 mutant mice, implying a regulation of CST by netrin-1, at least in part, via the Unc5h3 and DCC receptors. The role of Unc5h3 in axonal guidance has been very well established for the dorsal guidance of various axonal tracts in the developing hindbrain (Kim, D. et al., 2011).

Furthermore, in Un5h3 mutants with a B6 background severe defects in the projections of the trochlear nerve and the proper diaphragm innervation by the phrenic nerve have been detected, both of which are not present in other mutants related to the netrin-1 pathway (DCC, Ntnl or Neol) (Burgess, RW. et al., 2006). Unc5h3 is also expressed by dorsal root ganglia (DRG) neurons, dermamyotome, epidermis and ventral horn of the spinal cord during early developmental stages. This expression is thought to mediate the repulsion of DRG neurons from the ventral spinal cord where netrin-1 is highly expressed (floor plate). A similar defect is observed in the projection of early, but not late, DRG neurons in a Netrin-1 deficient environment (Masuda, T. et al., 2008). On the other hand, the transient expression of Netrin-1 in the dorsal spinal cord has also been shown to be important in the proper projection of DRG axons (Watanabe, K. et al., 2006).

\subsubsection{The emerging role of the DCC family receptor Neogenin}

Neogenin was identified as a close ortholog of DCC in vertebrates that can function as a netrin-1 receptor in axonal guidance. Although the expression of neogenin is widespread throughout the whole early embryo (E9.5), its CNS expression is still relatively low at E11.5, while by E13.5 there is an intense expression in the ventral part of the developing spinal cord. More specifically, in the neocortex neogenin expression can be detected at E13.5 at the outer most preplate and it remains strong in the upper cortical layers throughout the gestation period. In both 
the thalamus and the hypothalamus neogenin is weakly expressed at E13.5 but it increases as neurogenesis progresses. The hippocampal expression, however, remains high during the whole embryonic development (Gad, JM. et al., 1997). Contrary to DCC, neogenin appears to play a role in early neuronal differentiation since it is expressed in the ventricular zones of the neural tube, mesencepahlon, rhombencephalon and telencephalon (Keeling, SL et al., 1997). Specifically in the forebrain, neogenin protein has been shown to be present in the neuroepithelium of the E12.5 forebrain, the RGs of the VZ, as well as the SVZ and a population of migrating neuroblasts in the IZ, at E14.5, in a high lateral to low medial gradient. Additionally, interneuron neuroblasts that migrated out of the LGE and MGE were also neogenin positive (Fitzgerald, DP. et al. 2006). Except for embryonic neurogenesis, neogenin was also shown to play a similar role in the adult mouse and human forebrain (Bradford, D. et al. 2010). Within the pool of neural stem and progenitor cell (NSPC) population, neogenin is expressed in the continuously selfrenewing and neurogenic cells in the E11.5 cortex and the adult SVZ (Shoemaker, LD. et al. 2010).

Except for netrin-1, neogenin was shown to bind with even higher affinity than netrin-1 the relatively new chemorepulsive axon guidance molecules, called repulsive guidance molecules (RGM), in chick (Rajagopalan et al. 2004). RGMs were first identified as cues of temporal retinal axons of the chick tectum (Monnier et al. 2002). They can serve as both short and long-range cues since they are glycosylphosphatidylinositol membrane bound molecules that can also be cleaved to produce soluble ligands (Kuninger et al. 2006). The expression of neogenin strongly overlaps with that of RMGa (eg. high expression in the ventricular zones) while $\mathrm{RMGb}$ is mostly found in postmitotic cells, like DCC, and RMGc has no CNS expression. In addition to axon guidance, neogenin seems to also play a role in neuroepithelium integrity in zebrafish (Mawdsley et al., 2004) shown by defects in the pseudostratified neuroepithelium of the neural tube as well as by neural tube closure defects (exencephaly) in $R M G a$ loss of function mouse embryos (Niederkofler et al, 2004).

Similar to DCC, neogenin binds FAK kinase via its P3 domain and gets phosphorylated upon netrin-1 binding. However, unlike DCC, neogenin does not trigger FAK kinase phosphorylation. The RGMa triggered repulsion takes place via the activation of RhoA kinase (ROCK), through RhoA, which in turn phosphorylates 
the light chain of myosin II leading to F-actin depolymerization (Kubo et al., 2007). As far as the netrin-1 mediated attraction is concerned, neogenin is predicted to act in a similar to DCC way, namely through activating Rac1 and Cdc42 by FAK phosphorylation (De Vries et al., 2008).

\subsubsection{The newly identified Down syndrome cell adhesion molecule (DSCAM)}

DSCAM is a type one transmembrane orphan receptor that has been known for a while to be partially responsible for the cognitive deficits observed in individuals with Down's Syndrome (Yamakawa et al., 1998). Although the role of Drosophila DSCAM had been widely studied in the fly nervous system, only recently was there a report that implicated DSAM in the mammalian axon guidance. In a search for receptors with homology to DCC, Ly et al., (2008) identified DSCAM, which was expressed by commissural neurons of the developing mouse spinal cord and was shown to be required for the proper guidance of these axons to the ventral midline. Furthermore, netrin-1 was able to bind DSCAM both in vivo and in vitro at Ig domains seven to nine, with a dissociation constant similar to that reported for netrin1/DCC binding (Keino-Masu et al., 1996). Additionally, DSCAM forms a complex with DCC only in the absence of netrin-1, implying that the two receptors might signal independently.

A subsequent study by Liu, et al., 2009, reported similar results. DSCAM was shown to mediate attraction to netrin-1 in commissural neurons and to collaborate with DCC in netrin-1 mediated axon outgrowth. Moreover, the authors showed that DSCAM alone can mediate the phosphorylation of Pak1 and Fyn in response to netrin-1 and that DSCAM does not interact with DCC. Except for the commissural axons of the spinal cord DSCAM can also be detected in cell bodies and axons in the hindbrain of E10.5 mouse embryos (Andrews et al., 2008). 


\subsection{Scope of this work}

Some of the most important characteristics of the mammalian brain evolution have been the increase in cortical thickness and the development of the major axonal tract, the corpus callosum. Disorders involving corpus callosum development and the great variety of symptoms associated with them, ranging from asymptomatic cases to severe mental retardation, point to the redundant and compensatory mechanisms that dictate brain function. In our study, we are trying to identify the factors that play key roles in the specification of callosal projections and discern the molecular pathways via which these molecules exert their effect.

Several transcription factors have been shown to be important in the specification of the proper identity of cortical neurons both at the progenitor level and in postmitotic cells. Their mode of action, however, is for the most part still unclear. The alteration in the expression of various axon guidance molecules, which have been implicated in different ways in the formation of proper projections, upon the deletion of many neuron specific markers (transcription factors) suggests a role for these molecules in the acquisition of at least some aspects of the correct neuronal identity. We tried to elucidate in more detail the role of the axon guidance molecules that showed altered expression in the Satb2 $2^{-/}$mice and to dissect the pathway(s) involved in their regulation. To this end, we employed both genetic and overexpression methods.

The interplay between the known neuronal specific transcription factors has started to be partially revealed. We sought to identify the interactions amongst the laminar-specific genes (Satb2, Ctip2 and Fezl) and to dissect the ways by which these factors control the generation of callosal or subcortical projections. During the above effort, we came across various axon guidance molecules the role of which was proven to be important in the spocification of axonal projections. This role has been extensively studied for some molecules, as described above, in the broader context of axon guidance within the nervous system. In this study, based on the initial observation of the altered expression patterns of various axons guidance factors and the available information, before and during the study (presented in detail here), pertaining to their role in guiding neuronal axons in different contexts/organisms, we tried to dissect their contribution in guiding cortical axons during embryonic development of the murine forebrain. 


\section{MATERIALS AND METHODS}

\subsection{Mutant mice}

\subsubsection{Satb2 conventional Knockout mice}

The Satb2 knockout mice were generated in the lab as described in Britanova et al., 2006. The Cre recombinase sequence and a Neo expression cassette replaced the second coding exon of the Satb2 protein (Fig. 10). Homozygous mutants suffer perinatal lethality and can be easily recognized by the absence of the lower jaw. As discussed in Britanova et al., 2008, there were no differences observed between $\mathrm{Satb2}^{+/-}$and $\mathrm{Satb2} 2^{+/+}$embryos in terms of the position and number of Satb2 expressing neurons in the neocortex, therefore both $S a t b 2^{-/+}$and $S a t b 2^{+/+}$will be sometimes designated as wt animals.

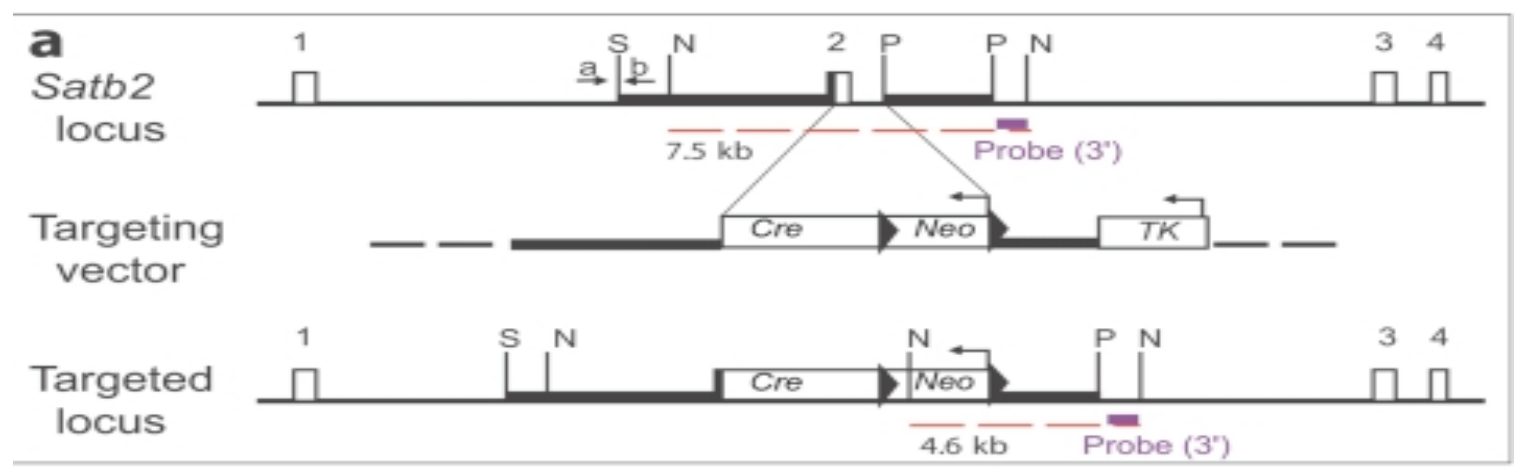

Fig. 10. Targeting of the Satb2 locus. Scheme depicting the insertion of the Cre gene and the neo cassette in the second coding exon of the Satb2 gene (Britanova, O. et al., 2006).

\subsubsection{Ctip2 (Bcl11b) conventional Knockout mice}

The Ctip2 null mice were generated in Dr. Kominami lab as described in Wakabayashi, et al., 2003. The neomycin resistance gene was inserted into exon 1 of the $B c l 11 b$ gene. Neonatal homozygous mice for the mutant allele die within the first day after birth and the majority of times are born with open, or partially open, eyes. 
Wild-type allele

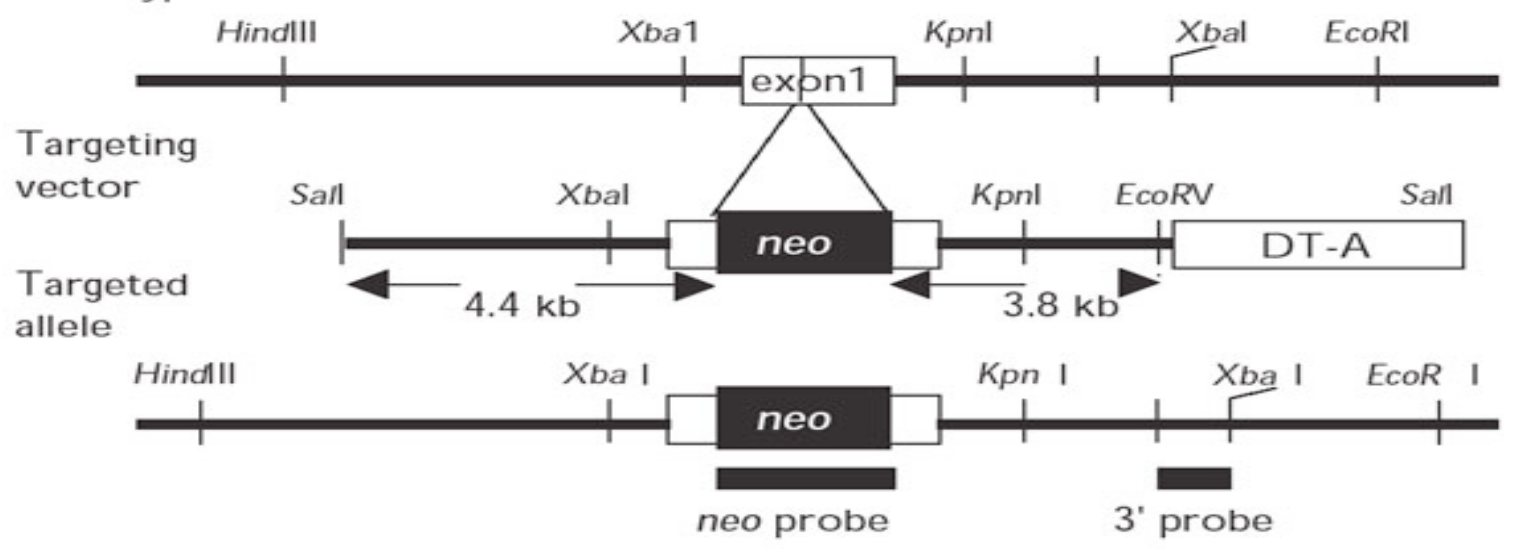

Fig. 11. Targeting of the Ctip 2 locus. The neo cassette was inserted in place of exon 1 in the $B c l 11 b$ locus (Wakabayashi, Y. et al., 2003).

\subsubsection{Fezf2 (Fezl) conventional Knockout mice}

The Fezl null mice were generated in Dr. Hibi lab as described in Hirata et al. (2004). A promoterless b-galactosidase gene and an MC-1 promoter linked neo gene replaced most of the coding region of the Fezl gene.

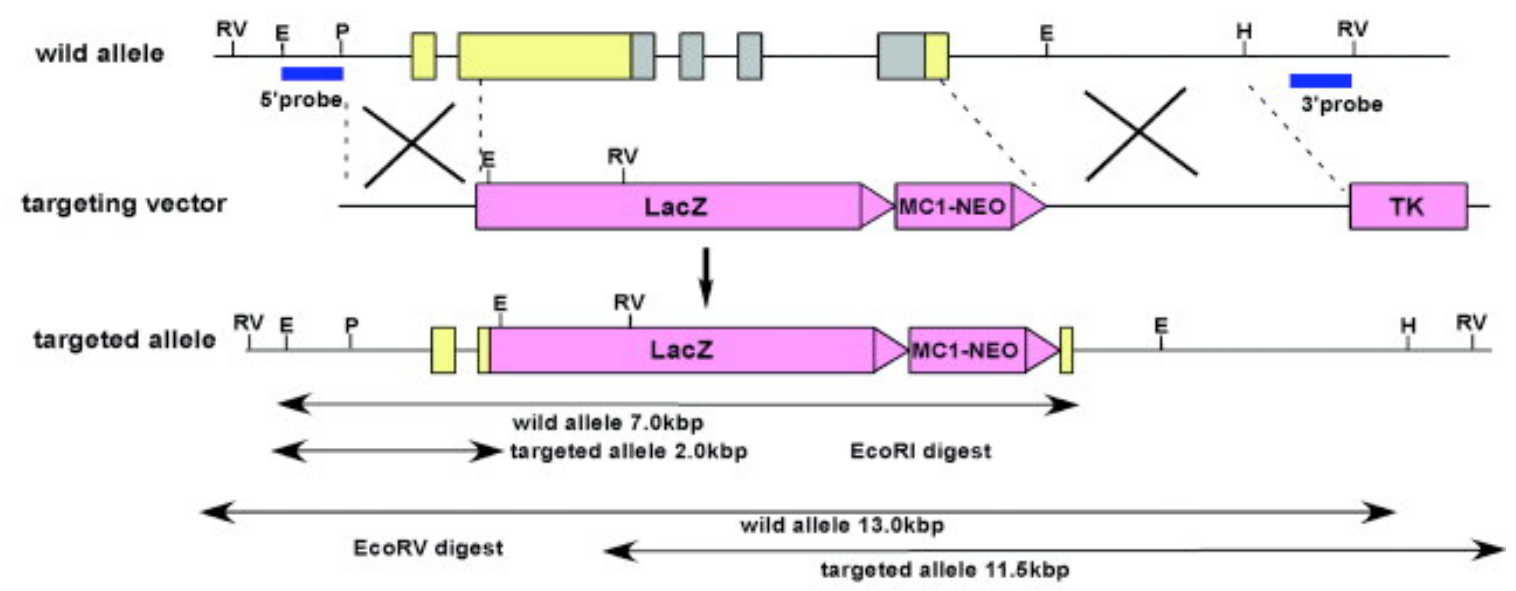

Fig. 12. Targeting of the Fezl locus. Scheme of the Fezl locus, the targeting vector and the targeted, mutant allele. The b-gal and neo genes have replaced most of the coding sequence of the Fezl gene (Hirata, T. et al., 2004).

In order to generate $\mathrm{Satb2}^{-/} ; \mathrm{Ctip}^{-/-}$or $\mathrm{Satb2}^{-/} ; \mathrm{Fezl}^{\gamma_{-}}$compound mutants, Satb2 $2^{+/-}$ animals were mated with $\mathrm{Ctip}^{+/-}$or $\mathrm{Fezl}^{-/+}$mice respectively. 


\subsection{Genotyping}

For genotyping purposes tails were cut either from embryos or from adult mice and digested @ $55^{\circ} \mathrm{C}$ for at least 2 hours (for embryos mostly overnight) in $300 \mu \mathrm{l}$ of lysis buffer $(100 \mathrm{mM}$ Tris- $\mathrm{HCl}$ pH8.5, 5mM EDTA, 200mM NaCl, $0.2 \%$ SDS, $100 \mu \mathrm{g} / \mathrm{ml}$ Proteinase K). In case of adult mice tails, lysis was followed by centrifugation at $13.000 \mathrm{rpm}$ for $5 \mathrm{~min}$ in order to get rid of hair and precipitate the DNA found in the supernatant with equal volume of isopropanol. After centrifgation for 10min@13.000rpm the DNA pellet was washed twice in 70\% ethanol and air dried for at least $15 \mathrm{~min}$ before finally resuspending in 50 to $200 \mu 1$ of sterile TE buffer (10 mM Tris-Cl, pH 7.5, 1 mM EDTA), depending on the pellet size.

The PCR volume was $20 \mu 1$ and the mixture had the following components:

10x Buffer (Genecraft) - $2 \mu 1$

$10 \mathrm{mM}$ dNTPs (Invitrogen) - $0.5 \mu \mathrm{l}(20 \mathrm{pmol} / \mathrm{ml})$

$10 \mathrm{nmol} / \mathrm{ml} \mathrm{Forward} /$ Reverse primers (IBA) - $1 \mu \mathrm{l}(40 \mathrm{pmol} / \mathrm{ml})$ each

TAQ polymerase (Genecraft/USB) - $0.5 \mu$ ( 0.5 units)

Template DNA - $1 \mu 1$

$\mathrm{ddH} 2 \mathrm{O}-14 \mu \mathrm{l}$

(All PCRs were performed with all 3 primers in the same reaction except Fezl wt and Lacz PCRs that were performed as two separate reactions)

The following protocols were used for each genotyping reaction:

1. Satb2 wt and mutant alleles ( $\sim 200 \mathrm{bp}$ and $\sim 450 \mathrm{bp}$ respectively)

Frw:ACCATCAGGCTGCTCAACC

Rvs:CAAGAGAGCCATCCAACTGC

CreRvs:CCAGACCGCGCGCCTGAAGA

Frw/Rvs=wt band, Frw/CreRvs=mutant band

$94^{\circ} \mathrm{C}-4^{\prime \prime}$

$55^{\circ} \mathrm{C}-20^{\prime \prime}$

$72^{\circ} \mathrm{C}-30$,

$\mathrm{X} 29$ cycles

$72^{\circ} \mathrm{C}-10$, 
2. Ctip2 wt and mutant alleles ( $\sim 550 \mathrm{bp}$ and $\sim 1300 \mathrm{bp}$ respectively)

Frw:AGAGGCCAAGCACTTTGAAGA

Rvs:TGCAGAGAAACTTTCCCAGAAC

NeoRvs:AGTGACAACGTCGAGCACAG

Frw/rvs=wt band, Frw/NeoRvs=mutant band

$95^{\circ} \mathrm{C}-1$,

$95^{\circ} \mathrm{C}-10$,

$60^{\circ} \mathrm{C}-20^{\prime \prime}$

$72^{\circ} \mathrm{C}-1{ }^{\prime} 30^{\prime \prime}$

X39 cycles

3. Fezl wt and mutant alleles ( 550bp and $\sim 200 \mathrm{bp})$

Frw:GCGAATTCGCTCCACGCTCAACACGCACATCCG

Rvs:CGGGATCCTTTAAGTCAAAGTTTCTGCAAAA

b-galFrw:ACGGCATGGTGCCAATGAATCGTCTG

b-galRvs:CAAATAATATCGGTGGCCGTGGTGTC

a) Fezl wt reaction:

$94^{\circ} \mathrm{C}-2^{\prime}$

$94^{\circ} \mathrm{C}-30^{\prime \prime}$

$60^{\circ} \mathrm{C}-30$ ',

$72^{\circ} \mathrm{C}-30$ ',

X34 cycles

$72^{\circ} \mathrm{C}-2^{\prime}$

b) Lacz mutant reaction:

$94^{\circ} \mathrm{C}-2^{\prime}$

$94^{\circ} \mathrm{C}-5^{\prime}$,

$62^{\circ} \mathrm{C}-30$ '

$72^{\circ} \mathrm{C}-40^{\prime \prime}$

X32 cycles

$62^{\circ} \mathrm{C}-1$ '

$72^{\circ} \mathrm{C}-5^{\prime}$

All PCR products were analysed by a 1,5\% agarose gel electrophoresis at 90-120V. The TAE buffer (40mMTris- acetate, 1mMEDTA, $\mathrm{pH}$ 8) used contained $0.5 \mu \mathrm{g} / \mathrm{ml}$ 
ethidium bromide (Fulka), and the bands were visualised under UV light in a gel documentation system. The loading dye added to each PCR product was consisting of OrangeG dye $(0.2 \%)$ and sucrose $(40 \%)$. A standard DNA ladder (Invitrogen) was used ( $600 \mathrm{ng})$ to compare band sizes.

\subsection{Histological procedures}

\subsubsection{Tissue processing}

Pregnant females were sacrificed in a $\mathrm{CO}_{2}$ chamber and E14.5 or newborn pups by decapitation. P0 and E14.5 brains were dissected and immersion fixed in 4\% PFA (in PBS) overnight at $4^{\circ} \mathrm{C}$ followed by several washes in PBS. If brains were used for paraffin sections they were incubated overnight in $0.9 \% \mathrm{NaCl}$, followed by dehydration through a series of increasing ethanol concentrations $(50 \%$ through $100 \%$ ), and last by isopropanol. Incubation in toluol for at least $3 \mathrm{hrs}$ was followed by Paraplast wax (maintained at $60^{\circ} \mathrm{C}$ ) in two o/n incubations. Finally they were embedded in wax using plastic chambers. The slicing was done in a microtome (Leica) in $10 / 12 \mu \mathrm{m}$ thick sections that were mounted on silane- coated slides (Marienfeld Histobond). If brains were to be used for cryosectioning, after PBS washing they were cryoprotected in $20 \%$ sucrose for at least $6 \mathrm{~h}$ before being embedded in OCT (TissueTek). The sectioning $(12 \mu \mathrm{m})$ was done on a Cryotome (Leica) and the sectioned tissue was mounted on Superfrost Plus slides (MenzelGläser).

\section{3.2 Immunohistochemistry}

Immunohistochemistry experiments were performed as described in Britanova et al. (2008). Briefly, Brains were sectioned in $12 \mu \mathrm{m}$ thickness and the sections were dried @ R.T. for 3-4h before being washed twice in PBS (10min each) @ R.T. followed by blocking in $1 \% \mathrm{BSA}+1 \%$ Tween-20 for $1 \mathrm{~h}$. @ R.T. Incubations with primary antibodies were done overnight at $4^{\circ} \mathrm{C}$ and after washing 3 times (10min each) with PBS secondary Abs were applied on sections for $45 \mathrm{~min}$ at RT. When performing IHC on diI labelled sections, detergent was omitted from the blocking solution. Primary 
antibodies were prepared in the same blocking solution and added to the sections followed by incubation at $37^{\circ} \mathrm{C}$ for $1 \mathrm{~h}$ before incubating $@ 4{ }^{\circ} \mathrm{C}$ overnight. The primary antibodies used were Ctip2 (Abcam, 1:250), L1 (1:150, Millipore), Satb2 (lab generated, 1:1000), GFP (1:500, Rockland), ds-red (clontech, 1/30), Brn2 (1/200, Santa Cruz). Secondary antibodies used were either AlexaFluor-tagged secondary antibodies (1:500, Molecular Probes) or Dylight-tagged secondary antibodies (1:500, Jackson ImmunoResearch Laboratories).

\subsubsection{In situ hybridization (Chromogenic and Fluorescent)}

Brains (P0 or E14.5) were dissected and immersion fixed in 4\% PFA@ $9{ }^{0} \mathrm{C}$ overnight. Subsequently, the brains were put in a 20\% sucrose solution (in PBS) for 6h@ $4^{0} \mathrm{C}$ before being embedded in OCT Tissue-Tek (Takara). The brains were sectioned at $12 \mu \mathrm{m}$ thickness and dried at R.T. for at least $4 \mathrm{~h}$. The sections were post fixed for 20min in 4\% PFA (in DEPC-PBS) and washed two times in DEPC-PBS followed by Proteinase K $(20 \mu \mathrm{g} / \mathrm{ml})$ treatment for $1,5 \mathrm{~min}$. Sections were put in a $0,2 \%$ Glycine solution (in DEPC-PBS) for 5min and after washing in PBS (X2) they were post fixed again in $4 \% \mathrm{PFA} / 0,2 \%$ Glutaraldyde for $20 \mathrm{~min}$ followed by washing. Pre-hybridization was done for 1h. @ $66^{\circ} \mathrm{C}$ and the probe incubation was performed at the same temperature overnight. Next day the sections were washed in $2 \mathrm{XSCC}$ solution $(\mathrm{pH}=4,5)$ and RNAse treated $(20 \mu \mathrm{g} / \mathrm{ml})$ for $30 \mathrm{~min} @ 37^{0} \mathrm{C}$, washed again in 2XSCC followed by 3 washes @ 60 $0^{\circ} \mathrm{C}$ in 2xSCC/50\%Formamide for at least 30min each. Subsequently, sections were washed in KTBT solution and after blocking in $20 \%$ sheep serum (in KTBT) for $1 \mathrm{~h}$, they were incubated with an anti-DIG-AP antibody (Roche) 1/1000@ $4^{0} \mathrm{C}$ overnight. Next day several washes with KTBT and NT(M)T were performed before the incubation in the AP substrate solution NBTBCIP (Roche) until the appearance of the signal. All incubations were done at R.T. unless otherwise indicated.

For FISH the sections in the first day were acetylated for $10 \mathrm{~min}$ before prehybridization and the second day were treated with $1 \% \mathrm{H} 2 \mathrm{O} 2$ for 5 times, 8 min each, before the overnight incubation in anti-fluorescein-POD antibody (Roche) 1/100@ $4^{0} \mathrm{C}$. The third day a tyramide-biotin solution (Pierce, 1/400) was applied for $20 \mathrm{~min}$. and the $\mathrm{H}_{2} \mathrm{O}_{2}$ treatment was repeated. Incubation with Streptavidin-Alexa488 
antibody (Jackson labs, $12 \mu \mathrm{g} / \mathrm{ml}$ in PBS) was done for $15 \mathrm{~min}$ followed by an incubation with anti-DIG-POD (Roche, 1/100) for 3-4h. Application of TyramideDNP (Perkin Elmer, 1/60) was performed for 30min before applying the rat anti-DNP antibody (Invitrogen, 1/75)@ $4^{0} \mathrm{C}$ overnight. The in between washes were done with TNT solution and the rest of the incubations@ @.T. The last day a donkey-anti ratAlexa594 antibody (Molecular Probes, 1/500) was applied on the sections for $1 \mathrm{~h}$ followed by DAPI staining for $6 \mathrm{~min}$.

The DIG (Unc5h3, Ntr-1, DCC) and/or FITC (Satb2, Ctip2) labeled anti-sense riboprobes used were generated using the primers seen below.

The primers used for making the in situ probes are the following:

Unc5h3-Fw:TCACGCCAGCAGGTGGAGGAAC

Unc5h3-Rv:CATGCTATTTTCTGCACACTCTG

Satb2-Fw: GCGGGAGGCCCAACGGCGCCGTGGC

Satb2-Rv: CTCCTGGCACTTGGTTGCTGACACA

Ctip2-Fw: GTCTTCAAGAACTGTAGCAACCTGAC

Ctip2-Rv: AAAAGTCATTTAAGTGTTGGCTTCTT

DCC-Fw: TTATTAGTCGGACAGCAAAAGTTAC

DCC-Rv: TGAATACTTTTTAGACCTGAGTTGG

Netrin1-Fw: AAGACTGTGACTCCTATTGCAAGG

Netrin1-Rv: TTCTTGCACTTGCCCTTCTTCT

\subsubsection{Nissl Staining}

For Nissl staining only paraffin embedded brains were used. Slides were dewaxed in Histoclear followed by rehydration through a descending series of ethanol concentrations (100\% to $10 \%$ ). After washing (in $\mathrm{H}_{2} \mathrm{O}$ ) they were incubated in $50 \%$ potassium sulfite for $15 \mathrm{mins}$, washed in PBS and stained in a Cresyl violet solution $(0,5-1.5 \%$ in acetate buffer) for $25 \mathrm{mins}$. Sections were subbsequently washed in acetate buffer $(10 \mathrm{mM}$ sodium acetate, $10 \mathrm{mM}$ acetic acid in $\mathrm{H} 2 \mathrm{O})$ for $1 \mathrm{~min}$ and then put in Differentiation buffer $(0.14 \%$ glacial acetic acid) until the desired color intensity was achieved. The slides were rehydrated in ethanol series and air dried for a 
few minutes. Mounting was done in a Xylol- based hydrophobic mounting medium (Eukitt).

\subsection{DiI tracing}

After overnight fixation of the brains in 4\% PFA, single crystals of 1,1'-dioctadecyl3,3,3',3'-tetramethylindocarbocyanine perchlorate (DiI; DiIC18(3), Molecular Probes) were placed in the cerebral peduncle. To access the cerebral peduncle, the brain was transected coronally, rostral to the superior colliculus. Brains were incubated for two weeks in the dark at $37^{\circ} \mathrm{C}$ and were sectioned into $80 \mu \mathrm{m}$ thick sections using a Leica vibroslicer. The sections were stained with an antibody against Ctip2. Images were taken on an inverted Leica confocal microscope (TCS-SP2 AOBS), at a pinhole of $1 \mathrm{AU}$ and were made as Z-stacks of $13 \mu \mathrm{m}$ depth with a step interval of $2 \mu \mathrm{m}$.

\subsection{In utero injection and electroporation}

The procedure was carried out as previously described (Saito, 2006). Briefly, pregnant females were anesthetized using Isofluran. Before operation, the females were given an injection $(\sim 0,9 \mathrm{ml})$ of Temgesic $(0,03 \mathrm{mg} / \mathrm{ml})$ as a pain blocker. Embryos from either uterine horn were pulled out and the plasmid solution was injected into one lateral ventricle using a micropipette followed by 6 electric pulses of $33 \mathrm{~V}$ each in $1 \mathrm{sec}$ intervals. All plasmid concentrations were $3-4 \mathrm{mgr} / \mathrm{ml}$. Postoperation, the mice were returned to their cage until sacrificed at P0.

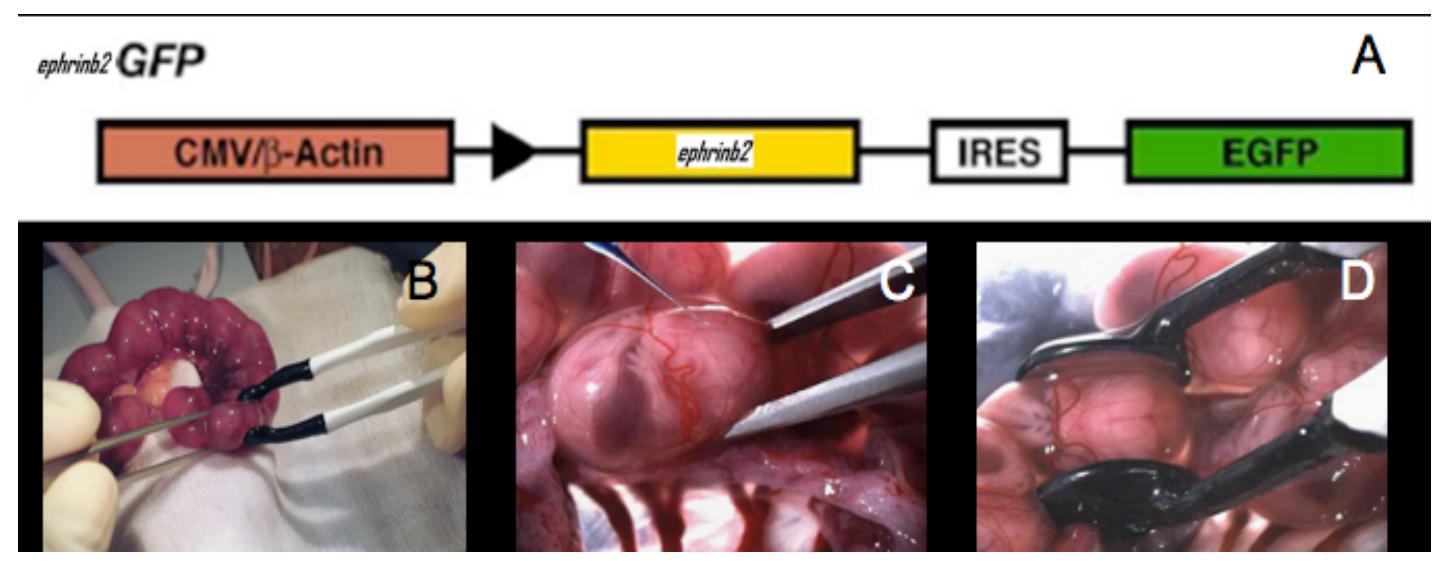


Fig.13 (A) Molecules used were cloned into vectors using CAG promoter and IRES GFP element. Pictures depicting the in utero electroporation procedure: (B) exposure of the uterus through incision in the abdominal cavity, $(\mathrm{C})$ injection through the uterine wall into the lateral ventricle of the brain and (D) electroporation with twizzer like electrodes

\subsection{Molecular biology methods}

\subsubsection{Transformation}

An aliquot of $50 \mu 1$ of E.coli DH5 $\alpha$ cells was thawed, mixed with 1-2 $\mu 1$ plasmid DNA and incubated on ice for $20 \mathrm{~min}$. The cells were permeabilised by heat shock at $42^{\circ} \mathrm{C}$ for $45 \mathrm{sec}$. followed by an incubation on ice for $3 \mathrm{~min}$. $500 \mu \mathrm{LB}$ medium was added to the cell mixture and cultured at $37^{\circ} \mathrm{C}$ for $1 \mathrm{hr}$ with agitation. $20-200 \mu \mathrm{l}$ of this mixture was then plated on LB-antibiotic agarose plates, and the plates were incubated inverted at $37^{\circ} \mathrm{C} \mathrm{o} / \mathrm{n}$. Individual colonies were picked, inoculated in $5 \mathrm{ml} \mathrm{LB}$ medium with the appropriate antibiotic and cultured overnight at $37^{\circ} \mathrm{C}$.

\subsubsection{Plasmid isolation}

The desired transformed cells were inoculated into $5 \mathrm{ml}$ LB medium with appropriate antibiotics, and cultured at $37^{\circ} \mathrm{C} \mathrm{o} / \mathrm{n}$ with rigorous agitation $(230 \mathrm{rpm})$. The cells were centrifuge precipitated at 6000rpm for 10min. QIAprep Spin Miniprep kit (Qiagen) was used to isolate the plasmid from the pelleted cells, according to manufacturer's instructions. The isolated plasmid was dissolved either in $\mathrm{ddH}_{2} \mathrm{O}$ or TE-buffer at desred concentrations.

\subsubsection{Plasmid linearization}

The purified plasmids containing the desired cDNA sequences were linearised using the appropriate restriction enzyme (New England Biolabs) in order to digest the sequence at the end opposite to the promoter used for probe synthesis (T3, T7 or Sp6). Around $5 \mu \mathrm{g}$ of plasmid DNA were digested each time. The appropriate buffer for each enzyme was used according to NEB instructions. The reaction mix was incubated at 
$37^{\circ} \mathrm{C}$ for $1,5 \mathrm{hrs}$ to overnight until complete linearisation was achieved. To test for the completion of the reaction a sample was run on an agarose gel.

\subsubsection{Purification of linearised plasmid DNA- phenol chloroform extraction}

Linearised DNA was precipitated by adding $1 / 10 \mathrm{~V} 3 \mathrm{M}$ sodium acetate $(\mathrm{pH}$ 5.5) follwed by rigorous mixing (vortexed). Tris-saturated phenol- chloroform/ isoamylethanol, pH8 (Invitrogen) was added in equal volumes. After centrifugation at $13,000 \mathrm{rpm}$ for $15 \mathrm{~min}$ the upper (aqueous) phase was transferred to a clean tube and $100 \% \mathrm{EtOH}$ was added to it (3X volume). DNA precipitation was accomplished by incubation at $-20^{\circ} \mathrm{C}$ for 1 hour to $\mathrm{o} / \mathrm{n}$, followed by centrifugation at $13,000 \mathrm{rpm}$ for $15 \mathrm{~min}$. The pellet was washed with $70 \%$ ethanol and air dried for $\sim 0,5 \mathrm{~h}$ followed by resuspention in $\mathrm{ddH}_{2} \mathrm{O}$ (DEPC when used for in situ)

\subsubsection{In vitro transcription of linearized plasmid (for cold in situ)}

For each reaction $\sim 2 \mu \mathrm{g}$ of linearized plasmid was used. The reaction mix was as follows:

plasmid $(\sim 2 \mu \mathrm{g})$

DIG labelling mix $(2 \mu 1)$

10X transcription buffer $(2 \mu \mathrm{l})$

RNA polymerase (Sp6, T3 or T7) $(2 \mu \mathrm{l})$

DEPC- $\mathrm{H}_{2} \mathrm{O}$ up to $20 \mu$ f final volume

The mixture was incubation at $37^{\circ} \mathrm{C}$ for $2 \mathrm{~h}$.

Addition of $2 \mu 1$ DNase (RNase free) was followed by incubation @ $37^{\circ} \mathrm{C}$ for 15 min. Precipitation was done by adding:

$2 \mu 10.2 \mathrm{M}$ EDTA $\mathrm{pH} 8.0$

$2.5 \mu 14 \mathrm{M} \mathrm{LiCl}$

\section{$75 \mu 1100 \% \mathrm{EtOH}$}

and incubating $@-20^{\circ} \mathrm{Co} / \mathrm{n}$. The next day the mixture was centrifuged for $30 \mathrm{~min}$ at $13.000 \mathrm{rpm}\left(4^{\circ} \mathrm{C}\right)$ to pellet the probe, which was then washed by adding $70 \mu 170 \%$ $\mathrm{EtOH}$, centrifuged again and left to air dry. Finally it was dissolved in 50 $\mu$ DEPC$\mathrm{H}_{2} \mathrm{O}$. The concentration was checked in a spectrophotometer (OD260) with 1/100 dilution and was also run on gel to check integrity. 


\subsubsection{Chromatin immunoprecipitation}

The cortical and basal ganglia tissues were isolated at $\mathrm{P} 0$.

The Chip assay was performed using Chip-IT express kit (Active Motif). The procedure was carried out according the manufacture's instructions. The following antibodies were used for Chip: rat monoclonal anti-Ctip2 (Abcam), polyclonal antirabbit IgG (Active Motif). The following primers were used:

Unc5h3 -60: Fw 5'-GGGGCAGAGCATTAGCAGC

Unc+100: Rv 5'- GGGGAAGGGAAACGCCACCA

GAPDH: Fw 5'- CAAGGCTGAGAACGGGAAG

GAPDH: Rv 5'- ATGGTGGTGAAGATGCCAGT.

The PCR was carried out using 33 cycles and an annealing temperature of $60^{\circ} \mathrm{C}$.

\subsubsection{Luciferase assay}

Expression plasmids were constructed by PCR techniques using proofreading polymerase Tersus (Evrogen).

Unc $5 \mathrm{~h} 3$ promoter regions were amplified with the following primers:

E1_EcoR1_Fw:5'TTAAGAATTCTTGCCTTCTTTCCCATCT, E1Rv:5'TATTGAGGATCCAGTGCTGGGAGGTGTAGCGC, E2Fw:5'TTAAGAATTCTCCTCCTCGGAGGCTC, E2Rv:5’TATTGAGGATCCTGAGACGCGCAAACAGCCGA.

The fragments were digested and cloned into pC1-TK-luciferase vector (Evrogen). The plasmid containing full length CTIP2 cDNA under CMV promoter was kindly provided by the Leid lab (Senawong T. et al., 2003). PC12 cells were cultured in DMEM with $1 \mathrm{~g} / 1$ glucose (BioWhittaker) and 5\% FCS (Invitrogen). Cells were transfected with Lipofectamine2000 (Invitrogen) according to the manufacture's instructions. For luciferase experiments transfected cells were analysed in 24 hours. Luciferase assays were performed in 96-well plates using the Dual-Luciferase kit (Promega). For standardization of transfection variability and increased readout stability, a mix of plasmids coding for renilla-luciferase (RL) was used (CMV-RL, 
TK-RL and SV40-RL; 1:2:10 molar proportions, respectively). DNA amounts were adjusted to equal levels with pcDNA3. Cells were treated with lysis reagent (Promega) according to the manufacturers instructions. Coupled firefly and Renillaluciferase assays were performed in a Lumat LB96V reader (Berthold Technologies). Results were given as Renilla-normalized relative luciferase units (RLUs) \pm standard deviation (SD). All experiments were repeated at least three times with a minimum of three replicates for each data point. All $p$ values were $\leq 0,05$. 


\section{RESULTS}

\subsection{Convergence of the Satb2 and the Netrin-1 pathways}

\subsubsection{Altered expression of axon guidance molecules in the $\mathrm{Satb} 2^{-/-}$}

Previous data from two intepended groups have shown that Satb2 is a key postmitotic determinant of callosal projection neurons (Britanova, O. et al., 2008; Alcamo, EA. et al., 2008). In Satb2 mutant mice the neocortical Cre expressing (Satb2-) neurons instead of projecting toward the midline to form the C.C. are misrouted into projecting either through the A.C., to the contralateral hemisphere, or to subcortical targets. Microarray data, previously obtained in the lab, had shown altered expression levels of various axon guidance molecules in the $S a t b 2^{-/-}$compared to wild type brains. In our efforts to investigate, in more detail, the nature of the above neuronal misguidance we decided to test for the mRNA expression patterns of various axon guidance molecules in both wt and Satb2 deficient mice. These experiments showed that the expression of many of them was altered, downregulated or upregulated, either in specific part of the cortex or throughout the whole CP (Table 1). The obtained results are partially overlapping with the altered expression patterns of many axon guidance molecules observed in a different Satb2 mutant (Alcamo, EA. et al., 2008).

\begin{tabular}{|l|l|l|l|l|}
\hline & Throughout CP & Superficial CP & IZ & Layer V \\
\hline upregulated & & $\begin{array}{c}\text { Efrnb2, Slit3, EphA5, } \\
\text { EphA7, EphB1, } \\
\text { Nrp1 }\end{array}$ & Sema6D, EphB6 & $\begin{array}{c}\text { Efrn2, Slit3, EphA5, } \\
\text { EphA7, EphB1 }\end{array}$ \\
\hline downregulated & $\begin{array}{c}\text { EphA4, PlexinA4, } \\
\text { Unc5h3 }\end{array}$ & & & Sema3C \\
& & & & \\
\hline
\end{tabular}


Table 1. Axon guidance molecules the expression of which is altered in the Satb2 deficient compared to the wt neocortex of P0 brains (data also included in doctoral thesis of Dr. Camino De Juan Romero and in Alcamo, EA. et al., 2008).

The expression of specific axon guidance molecules (EphA4, PlxA4 and Unc5h3) was almost abolished or severely reduced throughout the whole cortical plate of the mutant brains, an observation which led to the hypothesis that those molecules might play a key role in the axonal misguidance observed in the $S a t b 2^{-/}$, and therefore we decided to examine them more closely. As seen in Fig. 14 the expression of Unc $5 \mathrm{~h} 3$ is robustly downregulated in the CP of the Satb2 mutant brains. Interestingly, even though its expression is severely reduced in the cortex it remains unaltered in the basal ganglia where Satb2 is not normally expressed. This finding led us to the conclusion that Unc5h3 is a molecule that lies downstream of Satb2.

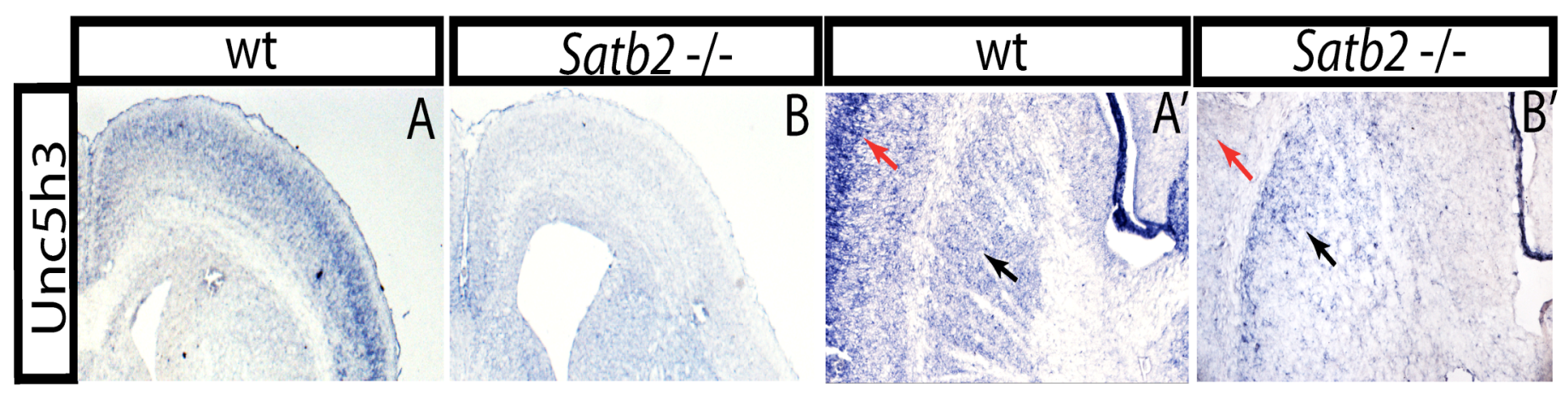

Fig. 14. Altered Unc5h3 mRNA expression in Satb2 deficient cortex. Unc5h3 expression in wt and Satb2 deficient brains assayed by chromogenic in situ hybridization. A and B: Unc5h3 mRNA in wt and mutant neonatal cortex respectively; A' and B': contrary to the cortex (red arrows), Unc5h3 expression in the basal ganglia (black arrows) is maintained in the mutant brains $(n \geq 4)$.

\subsubsection{Unc5h3 expression pattern is mostly overlapping with Satb2 and mutually exclusive with Ctip2 in the neonatal forebrain}

The observation that Unc5h3 expression is almost completely absent from the Satb2 deficient cortex, combined with the known role of Unc5h3 in mediating axonal repulsion to Netrin-1 signals (Kennedy et al., 1994; Ackerman et al., 1997; Hong, K. et al., 1999; Finger et al., 2002; Guan K.L. and Rao Yi, 2003), led to the hypothesis 
that callosally projecting neurons might need Unc5h3 expression in order to be repelled from the basal ganglia which is the main source of Netrin-1 in the forebrain. In this way, callosal neurons could be prevented from projecting subcortically and would instead be directed toward the midline. To examine whether Unc $5 \mathrm{~h} 3$ is indeed expressed by callosal projection neocortical neurons, double fluorescent in situ hybridization on neonatal wt brains was performed. As seen in Fig. 15, the mRNA expression of Unc5h3 is greatly overlapping with Satb2 and mutually exclusive with Ctip2 in the neocortex. Both Unc5h3 and Ctip2 are also expressed in the B.G. where their expression patterns are mutually exclusive as well (Fig. 15G-I). Furthermore, closer examination of the Unc5h3 and Satb2 mRNA cellular localization revealed that even in cases where they do not colocalize they still seem to be in the same cells, albeit in different subnuclear compartments (Fig. 15J). It is also noteworthy that the lateral boundary of Unc5h3 expression in the neocortex coincides with the dorsal boundary of Ctip2 expression in the superficial piriform cortex, an observation that further confirms their non-overlapping expression patterns (Fig. 16).

Fig. 15. Mostly overlapping expression of Unc5h3 with Satb2 and mutually exclusive with Ctip2. Double fluorescent in situ hybridization for Unc5h3 (red) and Satb2 or Ctip2 (green) in P0 wt brains. A-C Unc5h3 and Satb2 show a mostly overlapping expression pattern, while Unc5h3 and Ctip2 are mutually exclusive in both the cortex (D-F) and the basal ganglia (G-I). Higher magnification pictures of slides stained with DAPI in which it can be seen that the mRNAs of Satb2 and Unc5h3 can mostly be found in the same cells, although in different subnuclear compartments (J), whereas Unc5h3 and Ctip2 mRNAs have a mutually exclusive pattern in both the cortex $(\mathrm{K})$ and the basal ganglia $(\mathrm{L})$. 

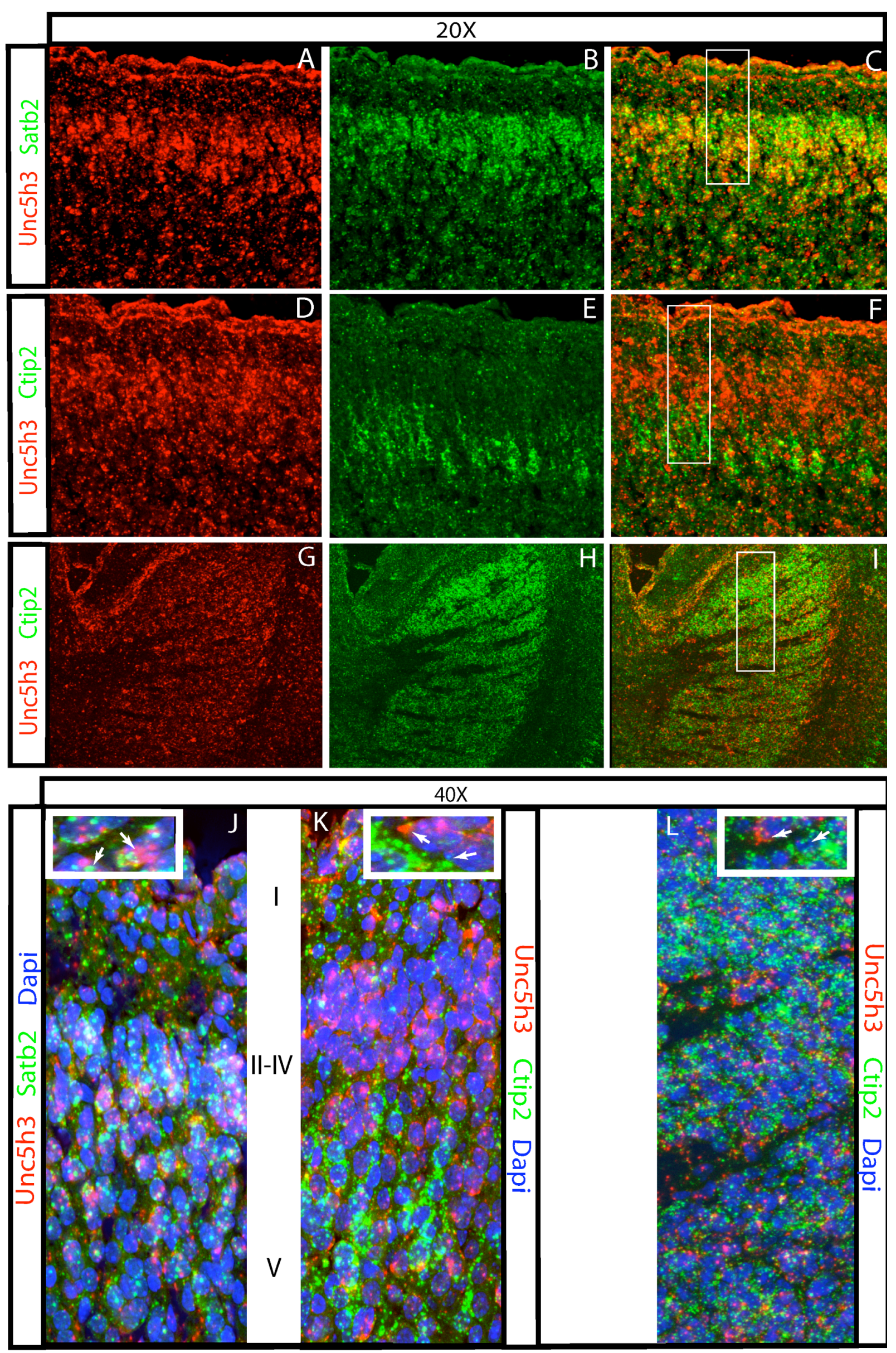


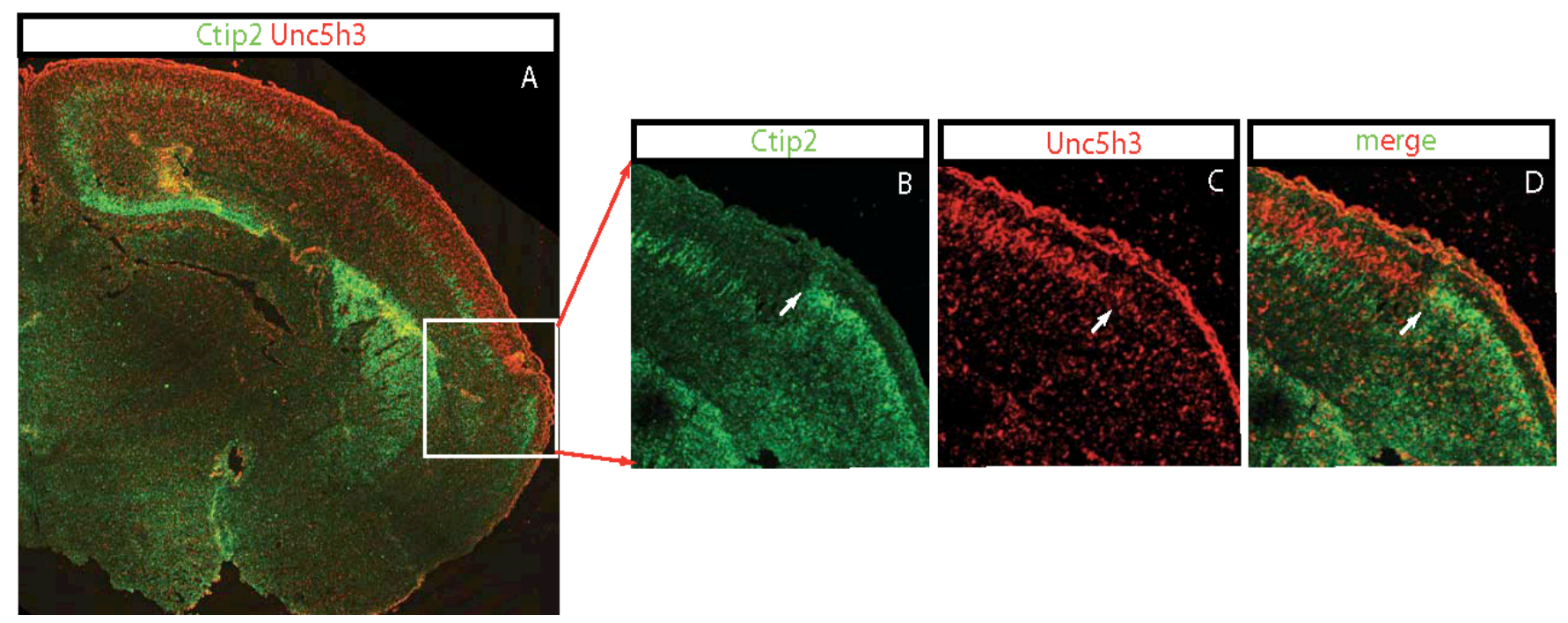

Fig. 16. The non-overlapping expression patterns of Unc5h3 and Ctip2 in the cortex. Double fluorescent in situ hybridization for Unc5h3 (red) and Ctip2 (green) in P0 wt brains (A). (B-D) higher magnification pictures of the boxed area in (A). The dorsal boundary of Ctip2 expression in the superficial piriform cortex (B) coincides with the lateral boundary of Unc5h3 expression (C) in the neocortex (D). White arrows point to the sharp border between their non-overlapping expression patterns.

\subsubsection{Unc5h3 expression is fully restored and callosal projections partially rescued in the $\mathrm{Satb2}^{--}$; Ctip $^{-/}$compound mutants}

Thus far, we have shown that Unc5h3 expression is mostly overlapping with Satb2 and mutually exclusive with Ctip2 and that it is almost completely abolished in the Satb2 deficient cortex. The above data, combined with the fact that in the Satb2 mutants there is an upregulation of Ctip2 expression in the Satb2 deficient neurons (Fig.17 and Britanova et al., 2008; Alcamo et al., 2008) led to the hypothesis that Ctip2 might be normally repressing Unc5h3 expression. To test this hypothesis, $\mathrm{Satb}^{-/} ;$Ctip $^{-/}$compound mutants were generated by crossing double heterozygote $\mathrm{Satb2}^{+/} ; \mathrm{Ctip}^{+/-}$animals. Examination of Unc5h3 expression in the mutant cortices showed that even though it was almost completely lost upon Satb2 deletion, it was fully restored after the additional deletion of Ctip2 (Fig. 18A-D).

These data are indicative of a repression exerted by Ctip2 on Unc $5 \mathrm{~h} 3$. 


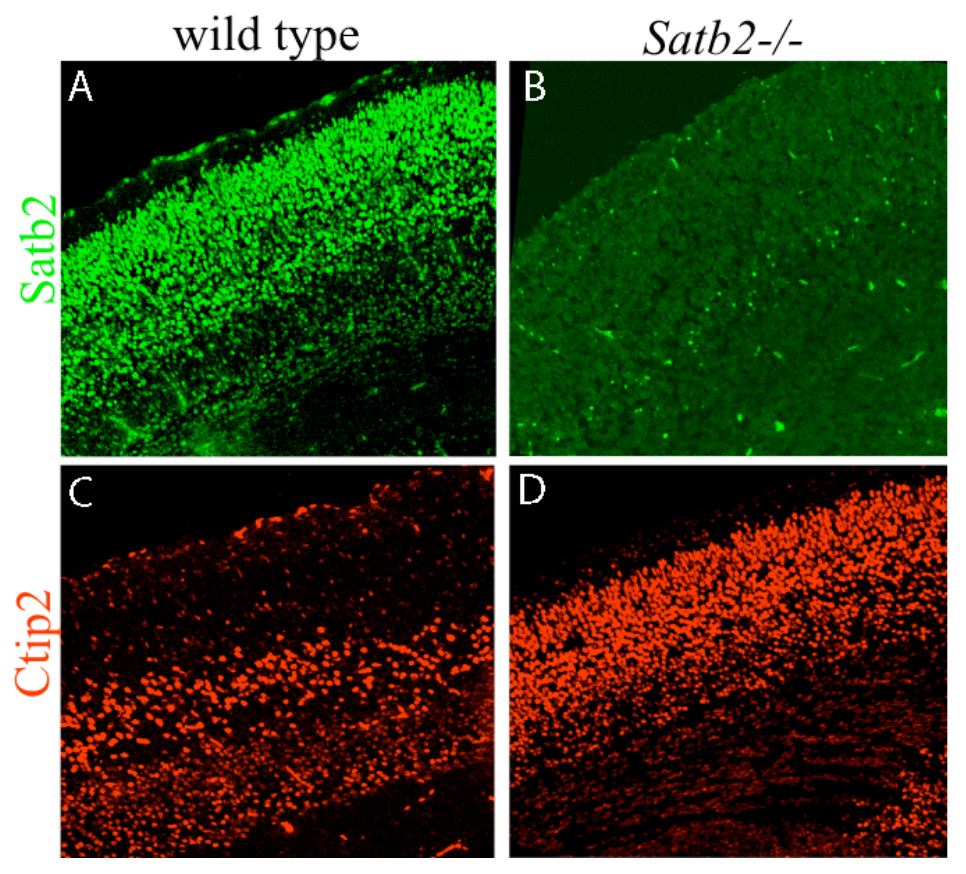

Fig. 17. Ectopic upregulation of Ctip2 in the superficial neocortex of the Satb2 ${ }^{-/}$. Satb2 (A and B) and Ctip2 (C and D) expression in the P0 cortex of wt and Satb2 $2^{-/}$brains assayed by immunohistochemistry. Satb2 expression is lost from the Satb2 mutant cortex, while Ctip2 is ectopically expressed in the area (UL) where Satb2 is normally found.

Following the observation that Unc5h3 expression is restored in the $S a t b 2^{-/-}$ ;Ctip $2^{-/}$compound mutants, the issue of determining the fate of callosal projection neurons in these animals needed to be addressed. For the above purpose, histological analysis with Nissl staining (Fig. 18E-H) as well as immunohistochemistry for the NCAM neural adhesion molecule L1, which labels most growing axons, were performed (Fig. 18I-L). This analysis gave the indication of a partial restoration of callosal projections in the Satb2 $2^{-\alpha} ; \mathrm{Ctip}^{-/}$. In order to confirm this result and to examine in more detail the axonal trajectories in the mutant embryos, in utero electroporation, at E12.5, of a plasmid encoding for a membrane bound Venus-GFP protein driven by a CAG promoter (gift of Hadjantonakis) was performed. These experiments revealed a considerable amount of axons projecting callosally in the $\mathrm{Satb2}^{-/} ; \mathrm{Ctip}^{-/}$, some of which were seen to cross the midline, in the most ventral part of the presumptive C.C., while others were projecting deep into the septum (Fig. $18 \mathrm{P}$, note the white arrow in picture inset). These data show that Unc5h3 expression is negatively regulated by Ctip2 and that the callosal projections that were completely

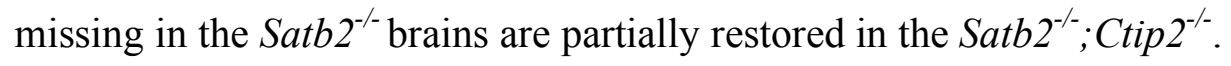



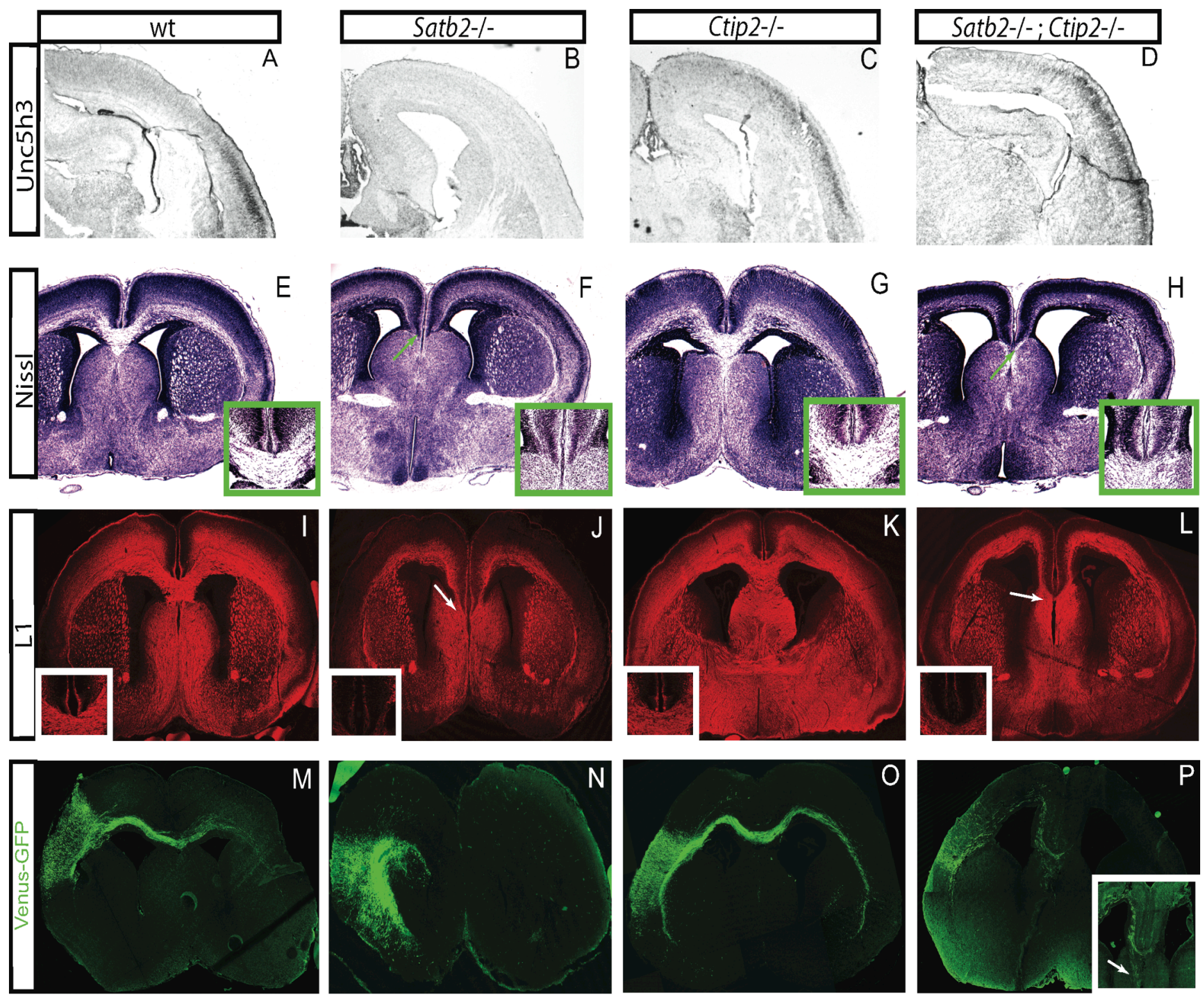

Fig. 18. Complete restoration of Unc5h3 expression and partial rescue of callosal projections in the Satb2 ${ }^{--}$; Ctip2 $^{-/}$. (A-D) Chromogenic in situ hybridization for Unc5h3 in wt brains and in the three different mutant genotypes indicated. Results show a complete restoration of Unc5h3 expression in the compound mutants $(n=2)$ that was missing from the Satb2 $2^{-/}$brains $(n \geq 4)(E-H)$ Nissl staining shows a partial restoration of callosal projections in the $\mathrm{Satb2}^{-/} ; \mathrm{Ctip}^{-/-}$(green arrows), indicated also with immunohistochemistry for the N-CAM molecule L1 (I-L, white arrows). (M-P). In utero electroporation, at E12.5, of Venus-GFP confirmed the partial restoration of callosal projections in the compound mutants. While all axons project subcortically in the Satb2 ${ }^{-/}(n \geq 3)$, a considerable amount of neurons that follow callosal trajectories can be seen in the compound mutant (inset in P shows that a certain number of axons cross the midline while others project deep into the septum (white arrow)). 


\subsubsection{Exogenous introduction of Unc5h3 into the Satb2 deficient neocortex can partially restore the missing callosal projections in a stage specific manner}

The partial callosal restoration observed in the $\mathrm{Satb2}^{-/} ; \mathrm{Ctip}^{-/-}$compound mutants, which also regain Unc5h3 expression, urged us to examine whether we could achieve a similar partial rescue in callosal projections by exogenously introducing Unc5h3 back into the Satb2 deficient neocortex. To test this hypothesis, in utero co-electroporation of a plasmid in which the expression of Unc5h3 is driven by a CAG promoter (Powell et al., 2008) along with the CAG driven, membrane bound VenusGFP reporter (same as above) was performed, in order to visualize the axonal projections of the Unc5h3 overexpressing neurons. The embryos were electroporated at E12.5 so as to influence both deep and upper layer callosal neurons. As seen in Fig. 19, overexpression of Unc5h3 can partially restore the callosal projections that were completely missing from the $S a t b 2^{-/-}$brains by directing some of the axons to project toward the midline (Fig. 19D). As expected, injection of just Venus-GFP showed that axonal projections in the Satb2 mutant brains are directed only to subcortical targets (Fig. 19B).

We next wanted to examine whether introduction of Unc5h3 into the Satb2 deficient brains in later developmental stages, e.g. at E14.5 when the majority of upper layer neurons are born, would have the same effect on axonal projections. In order to address this question, in utero electroporation was performed at two different developmental stages. First, co-electroporation was done at E12.5 of the Unc5h3 expression plasmid used above, along with the VenusGFP reporter (green), followed by a second electroporation at E14.5 of a plasmid (gift of Srinivas, S.; Trichas, G. et al., 2008) that encodes for a CAG driven, membrane bound td-tomato protein (red) in the same wild type and mutant embryos. All brains were isolated and examined at P0. With this strategy it was possible to trace the axonal projections of the Unc $5 \mathrm{~h} 3$ overxpressing neurons born at E12.5 onwards which were labeled green, and those of neurons born after E14.5, which were also labeled red. During analysis of the above electroporated wt and mutant brains it was observed that in the Satb2 mutants the Unc5h3 overexpressing neurons born after E14.5 sent axons only to subcortical targets (red), whereas many of those born between E12.5 and E14.5 (green) sent axonal projections toward the midline (Fig. 19H-J and N-P). In other words, the neurons whose axons can adopt callosal trajectories in the Satb2 deficient brains are 
born in a developmental time window between E12.5 and E14.5. Contrary to what is observed in the Satb2 $2^{-/}$brains, the wt neurons that are born after E14.5, and normally populate the upper cortical layers, direct their axons (red) toward the midline to form the C.C. (Fig. 19E-G and K-M, red axons). Interestingly, these axons are seen crossing the midline in the dorsal part of the C.C., known to be formed by later born $\mathrm{CPN}$, whereas axons of neurons that are born earlier are seen crossing the midline in the most ventral part (green).

Similar to what was observed in the E14.5 electroporated brains, overexpression of Unc5h3 at E16.5 failed to restore any callosal projections, since most of the neurons injected at this stage did not seem to migrate into the cortical plate (Fig. 20A-C). The Satb2 mutants can be easily identified after birth by striking phenotypic characteristics like microcephaly and micrognathia (Britanova et al., 2006). In addition to standard genotyping and phenotypic characterization, staining for Ctip2 was also performed in order to detect the Ctip2 upregulation in the mutant cortex and thereby further confirm the mutant genotype (side panels in pictures of Fig. 19 and 20).

Fig. 19. Partial restoration of callosal projections in the $S a t b 2^{-/}$by Unc5h3 injection at E12.5. (A and C) In utero electroporation of Venus-GFP at E12.5 in wt (A) and Satb2 mutant $(\mathrm{n} \geq 2)$ brains (B), where it is shown that mutant axons project only to subcortical targets. Unc5h3+Venus-GFP injection in wt $(n=2)(C)$ and $\operatorname{Satb2}^{-/}(n \geq 6)(D)$ where it is shown that some axons in the mutant brains are directed to project toward the midline. Injections of Unc5h3+Venus at E12.5 and ds-red at E14.5, in the

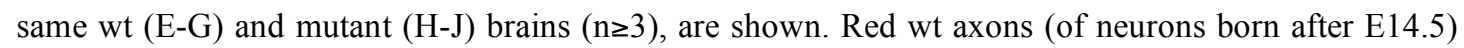
are seen crossing the midline in the dorsal part whereas green wt axons (of neurons born between E12.5 and E14.5) are seen crossing in the most ventral part. In the Satb2 $2^{-/}$brains the red axons are directed only to subcortical targets whereas the green axons are guided toward the midline (partial rescue in the mutant attributed to neurons born between E12.5 and E14.5). K-M are higher magnification pictures of the wt, and N-P of the mutant double electroporated brains. The side panels in the pictures show immunohistochemistry for Ctip2 (red) for further confirmation of the mutant genotype by detecting the Ctip2 upregulation in the Satb2 mutant cortex. 

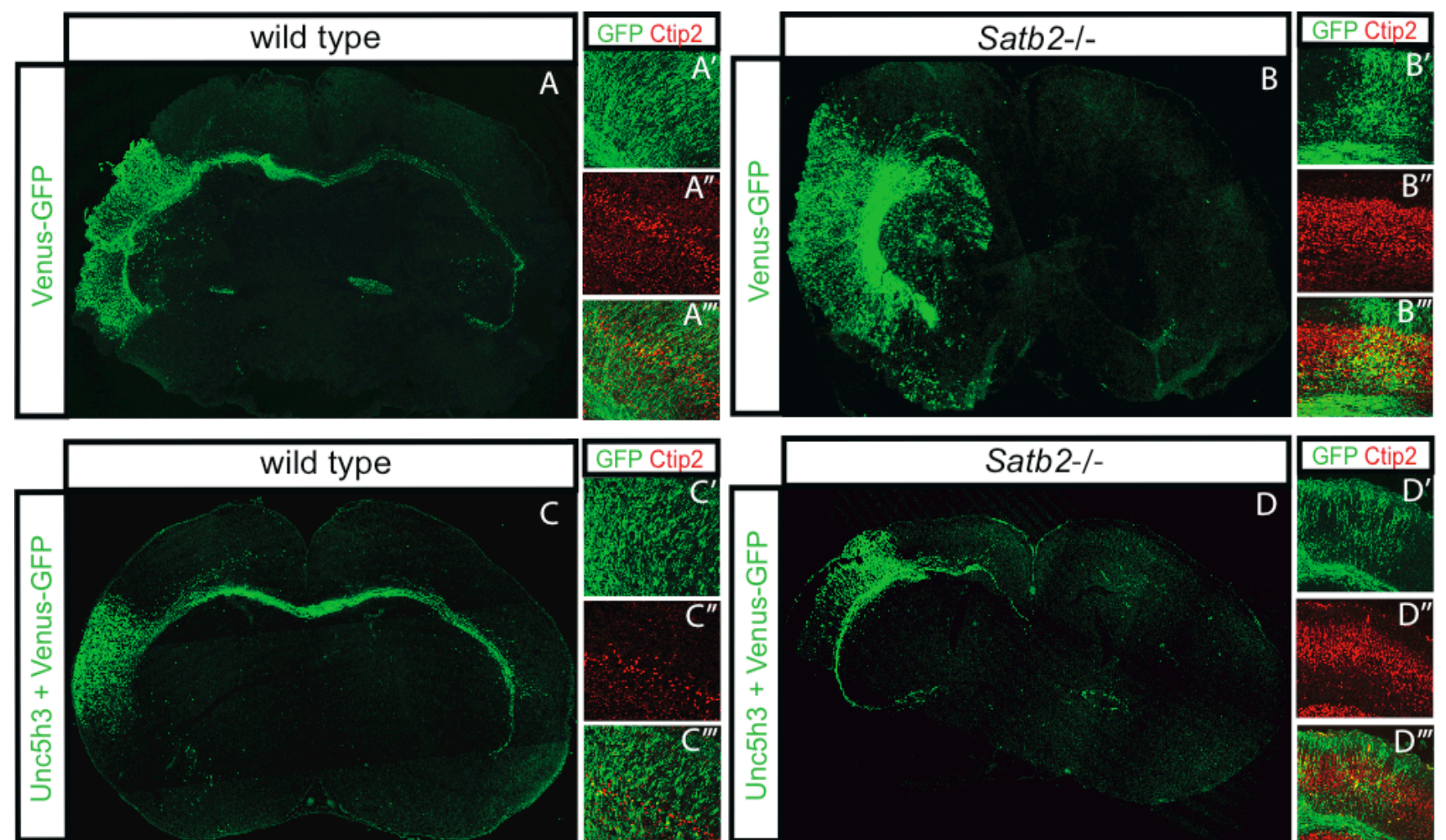

Satb2-/-

Td-tomato
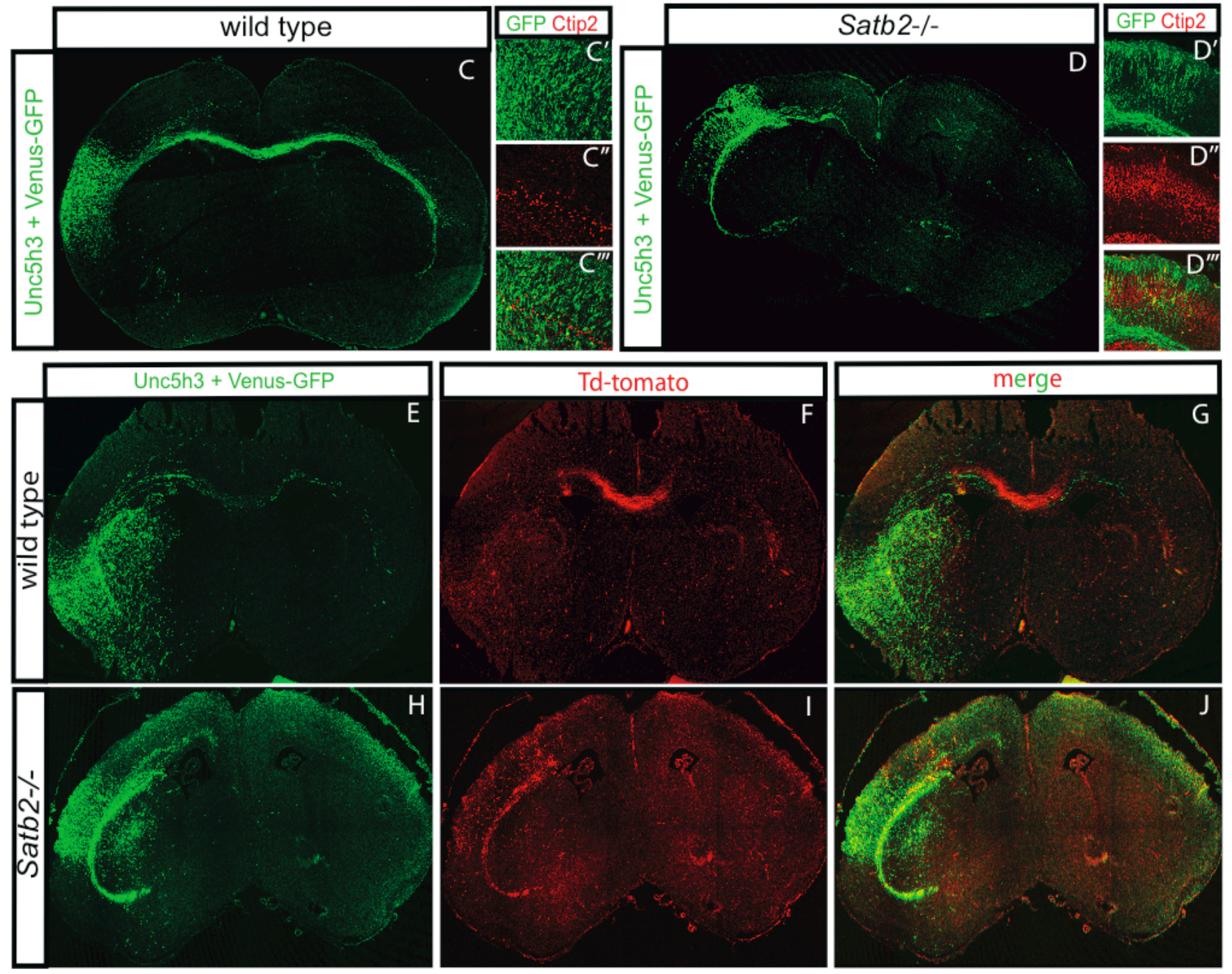

E
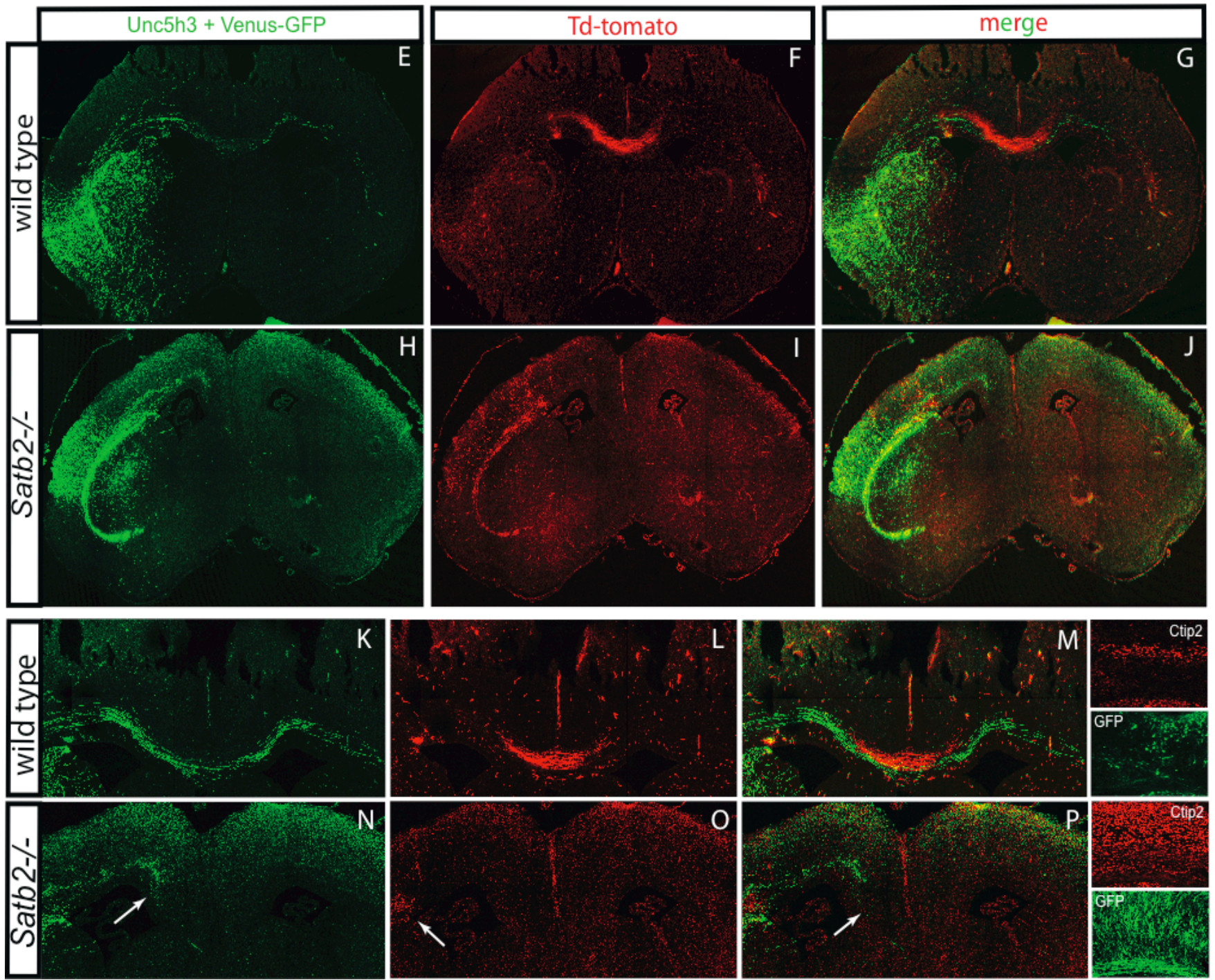
Although in the majority of electroporated mutant brains $(n \geq 6)$ we observed a partial rescue similar to that seen in Fig. 19D and H, there were also some exceptions. In some instances $(\mathrm{n}=2)$, where the electroporated mice delivered prematurely $(\sim 0,5-1$ day) there was no considerable number of axons observed approaching the midline whereas in one case of full gestation even more axons were seen to be directed toward the midline (data not shown). It should be noted that even in the cases where axons are seen to adopt a callosal trajectory, few of them cross the midline, indicating retardation in axonal extension. However, upon Unc5h3 overexpression these labeled axons that aquire the partial callosal identity are seen to turn medially and project through the ipsilateral cingulate cortex (and not laterally to project through the I.C.) going down into the corticoseptal boundary (Fig 19D), something which is not observed when mutant brains are injected with Venus-GFP alone (Fig 19B). It is also interesting to note that a similar retardation in axonal extension is observed in the axons that give the partial callosal rescue in the Satb2 $2^{--} ; \mathrm{Ctip}^{-/-}$brains as well (Fig 18P). These axons, even though projecting to the midline, do not manage to reach the appropriate targets in the contralateral cortex by $\mathrm{P} 0$.
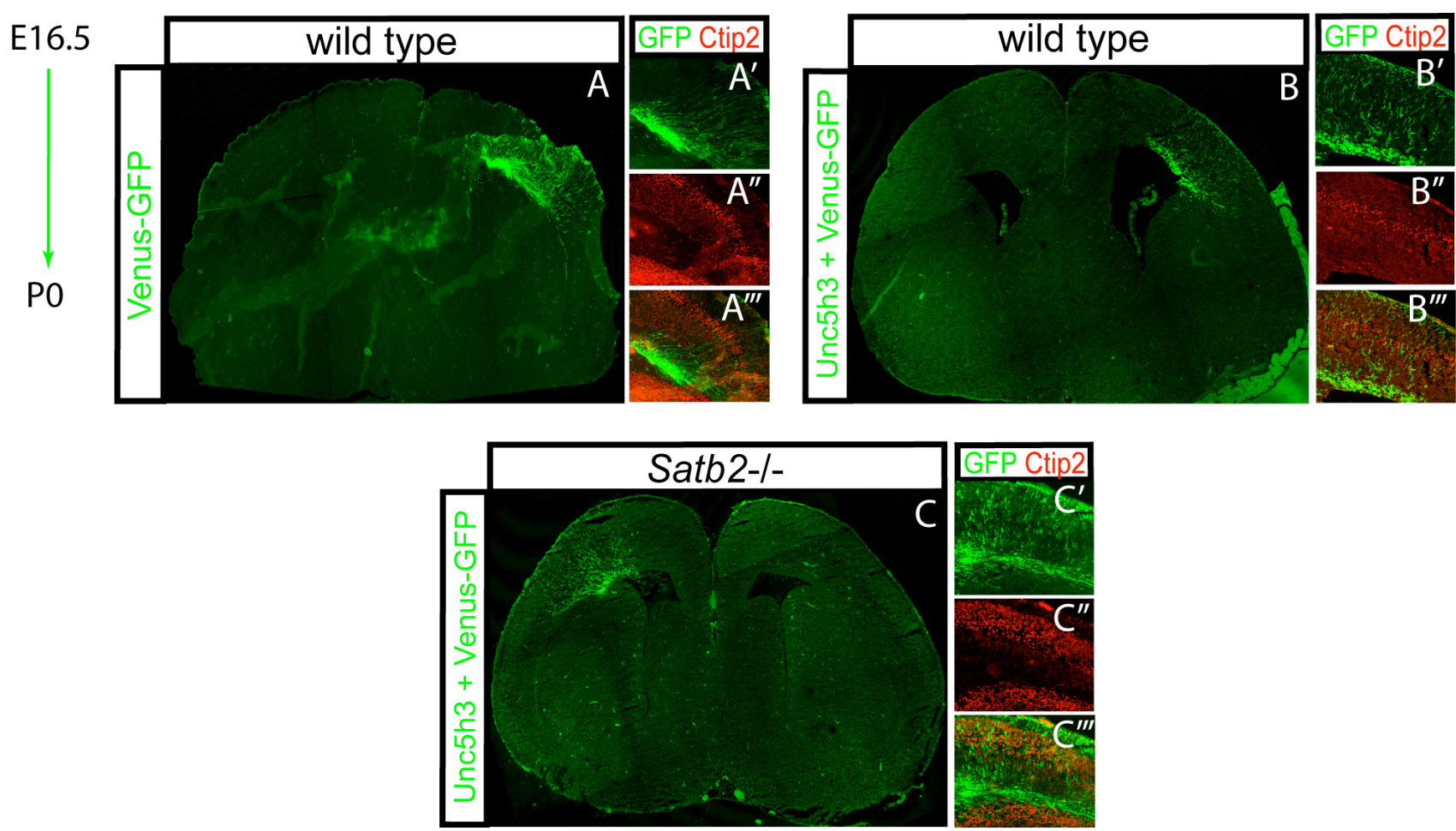

Fig. 20. Unc5h3 in utero electroporation at E16.5 does not result in any callosal rescue in the Satb $^{-/}$. Unc5h3+Venus-GFP electroporation at E16.5 in wt (B) and Satb2 mutant (C) brains (n=2). No 
rescue of callosal projections was observed in the mutant brains upon Unc $5 \mathrm{~h} 3$ overexpression, since even in wt brains injected with just Venus-GFP (A) the labeled neurons did not seem to migrate to the cortical plate at this stage.

It is noteworthy, that the expression of two other axon guidance molecules that are greatly reduced in the Satb2 ${ }^{-/}$(PlexinA and EphA4) is not restored after the additional deletion of Ctip2 in the Satb2 $2^{-/}$, contrary to what was observed for Unc5h3. As depicted in (Fig. 21E-H) the expression of both EphA4 and PlxA4 is missing from the Satb2 $^{-/}$; Ctip $^{-/-}$mutant neocortex similar to what was seen in the Satb2 mutant brains. Collectively, the above data point to the important role of specifically Unc5h3 in delineating callosal identity and thereby in directing axons to project toward the midline.
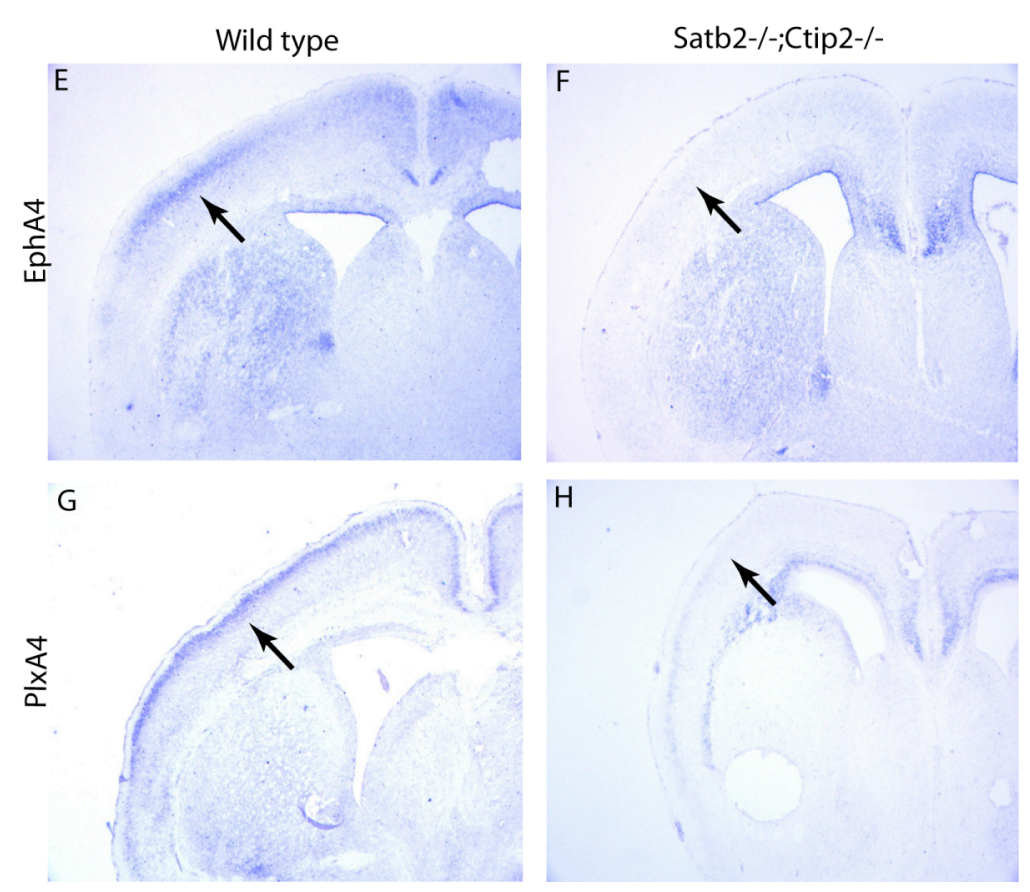

$\mathrm{H}$

Fig. 21. Expression of both EphA4 and PlxA4 is not restored in the compound mutants. In situ hybridization for EphA4 in wt (E) and Satb2 ${ }^{-/} ; \mathrm{Ctip2}^{-/-}(\mathrm{F})$ brains showing that its expression is missing from the cortex of compound mutants. A similar lack of restoration in the Satb2 $2^{-/} ; \mathrm{Ctip}^{-/-}$, is also observed for the neocortical expression of PlxA4 $(n=3)(G$ and $H)$. 


\subsubsection{Upregulation of DCC expression in the cortex of both $\mathrm{Satb}_{2}^{-/}$and $\mathrm{Satb2}^{-/-}$ ;Ctip ${ }^{-/-}$deficient brains}

The fact that the callosal restoration observed in the Satb2 mutants upon Unc5h3 overexpression, as well as the similar restoration observed upon the additional deletion of Ctip2 (in the Satb2 $2^{-/} ; C t i p 2^{-/}$mutants) was only partial, albeit the full restoration of Unc5h3 expression in the $S a t b 2^{-/} ; \mathrm{Ctip}^{-/-}$, urged us to investigate the possible involvement of other factors that might play a role in the observed misguidance. DCC is another Netrin-1 receptor, which has been shown to mediate attraction to Netrin-1 signals (Keino-Masu K. et al., 1996) and to be important for commissural axonal guidance (Fazeli et al., 1997; Serafini, T. et al., 1996; Ren, T. et al., 2007). In situ hybridization for DCC in neonatal wt brains (Fig. 22E) showed a diffused low-level expression throughout the cortical plate, a pattern that is also observed in the Ctip2 mutant brains (Fig. 22G). Conversely, in the Satb2 $2^{-/}$ and $\mathrm{Satb2}^{-/} ; \mathrm{Ctip}^{-/-}$mutant cortices DCC expression is highly upregulated in the superficial part of the cortex (Fig. 22F and H) in a pattern which mirrors the normal Satb2 expression (Fig. 22I), indicating that DCC is likely to be repressed by Satb2. Interestingly, although DCC expression is elevated in the neocortex it remains unaltered in the hippocampus, where the expression of Satb2 is normally very low (Fig. 22F, $\mathrm{H}$ and I), giving a further indication that Satb2 is regulating DCC expression.

In situ hybridization experiments were also performed for netrin-1 in wt and all three different mutant brains. No noticeable change in its expression pattern was detected suggesting that neither Satb2 nor Ctip2 exert any effect on Netrin-1 expression. Collectively, the above data show that Satb2 and Ctip2 can regulate the neuronal response to netrin-1 signals by repressing the expression of DCC and Unc5h3 respectively.

Fig. 22. DCC is upregulated in the $\mathrm{Satb2}^{-/-}$and $\mathrm{Satb2} 2^{--} ; \mathrm{Ctip2^{-/- }}$ mutants, whereas netrin-1 remains unaffected. Chromogenic in situ hybridization for netrin-1 (A-D) and DCC (E-H) in P0 wt, Satb2 $2^{-/}$, $\mathrm{Ctip}^{-/-}$and Satb2 $2^{-/}$; $\mathrm{Ctip}^{-/-}$brains. Netrin-1 expression in the basal ganglia remains unchanged in all three mutants, whereas DCC expression is highly uprelulated in the superficial cortex of both $\mathrm{Satb2} 2^{-/-}$ and $\mathrm{Satb2}^{-/-} ; \mathrm{Ctip}^{-/-}(\mathrm{n} \geq 3)(\mathrm{F}$ and H). Expression of DCC in the hippocampus however, where Satb2 is 
not normally expressed at this stage (P0), remains unaffected. Panel (I) shows FISH for Satb2 (converted to black and white) and (J) DAPI staining of the same section (also converted to black and white) to show the extremely low levels of Satb2 expression in the hippocampus. Magenta arrows point to the borders of normal Satb2 expression (I) which mirrors the area of upregulation of DCC in the Satb2 $2^{-/}$and Satb2 $2^{-/-}$;Ctip $2^{-/-}$mutants. (all $\mathrm{n} \geq 2$ )
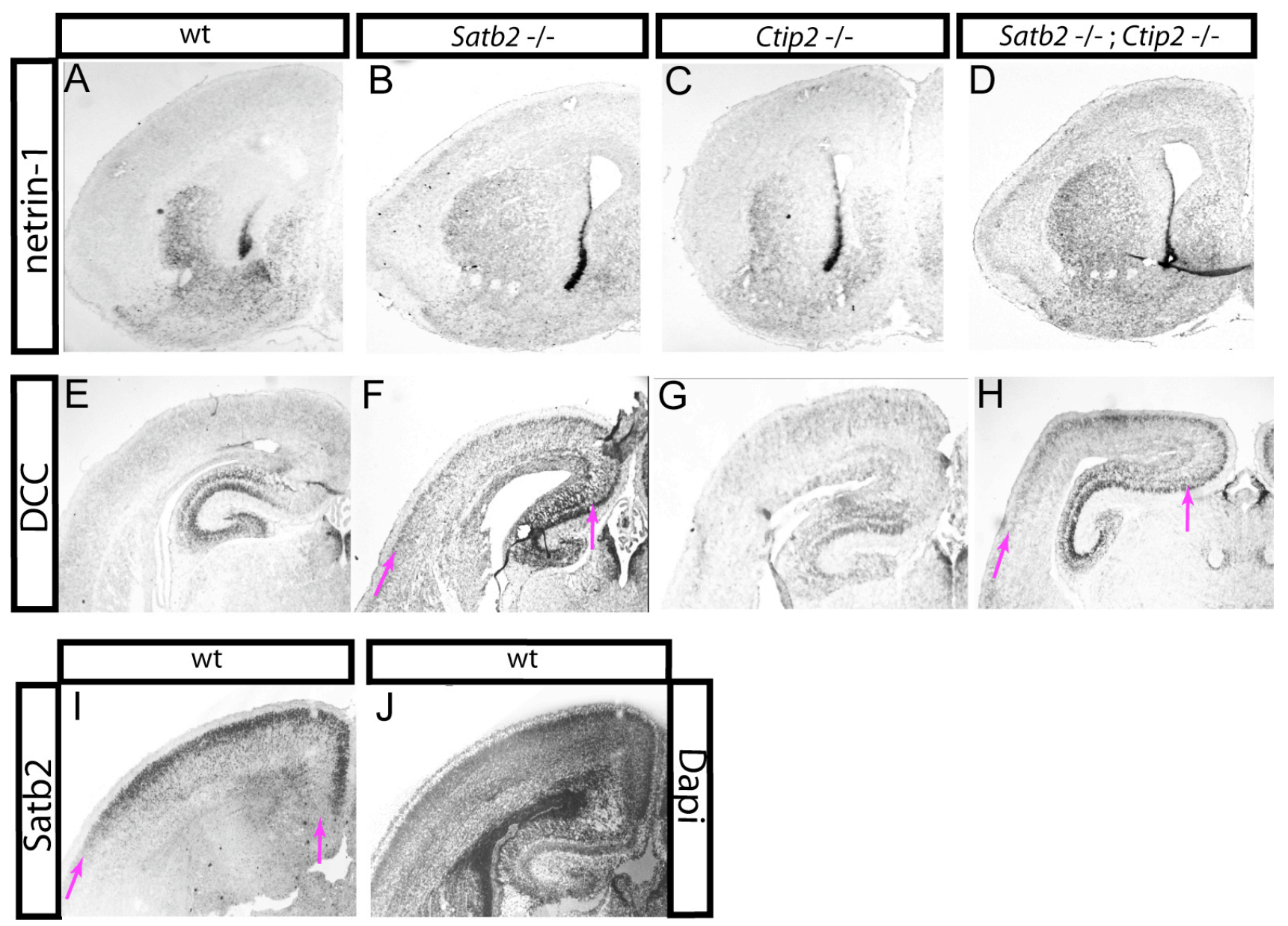

\subsubsection{DCC and Unc5h3 are dynamically regulated during embryonic development, and their expression at E14.5 is not affected by Satb2 and/or Ctip2 deletion}

We next sought to examine more precisely the role of the two netrin-1 receptors in regulating axonal guidance in the mouse forebrain. To this end, the 
expression of DCC and Unc5h3 was assayed by in situ hybridization at an earlier developmental stage (E14.5), in order to determine if and how their expression patterns change throughout development. In situ hybridization experiments showed that at E14.5, DCC is expressed in the cortex of both the wt brains and all three different mutants tested (Fig. 23A-D). Contrary to DCC, the expression of Unc5h3, although clearly evident in certain hypothalamic nuclei of the ventral forebrain, was not yet apparent in the cortex (Fig. 23I-L). At this stage (E14.5), most deep layer neurons are already born and have started sending their axons, through the internal capsule, to subcortical targets. Therefore, the presence of DCC is necessary in order to mediate attraction to netrin-1, the main source of which is the basal ganglia, thereby directing axons to project subcortically. On the contrary, Unc5h3 expression is not yet needed, given that upper layer callosaly projecting neurons have just started to be born.

As development progresses, DCC is downregulated in the cortex by Satb2 (Fig. 23E) while Unc5h3 is starting to be expressed at a time point after E14.5, and is quite prominent by P0 (Fig. 14A). Netrin-1 on the other hand, seems to have a steady expression in the basal ganglia, which is not influenced by Satb2 and/or Ctip2 deletion throughout development (Fig. 23E-H). These data show that there is a highly dynamic regulation of DCC and Unc5h3 expression during embryonic brain development. This spatiotemporally regulated expression of the two netrin-1 receptors ensures the appropriate, for each developmental stage, response to netrin-1 signals in order to in turn achieve the proper guidance of axons in the mouse forebrain (see discussion).

Fig. 23. No effect of Satb2 and/or Ctip2 on the expression of Netrin-1 or its receptors, DCC and Unc5h3, at E14.5. Chromogenic in situ hybridization for DCC (A-D), Netrin-1 (E-H) and Unc5h3 (I-

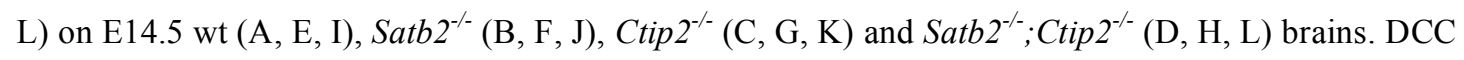
expression is evident in the cortical plate and medioventral forebrain and Netrin-1 expression is present in the basal ganglia and ventral VZ of both the wt brains and all three different mutants examined. On the contrary, Unc5h3 expression, although robust in various hypothalamic nuclei of the ventral forebrain, is not yet apparent in the cortical plate of all four genotypes examined ( $\mathrm{n} \geq 2)$. 

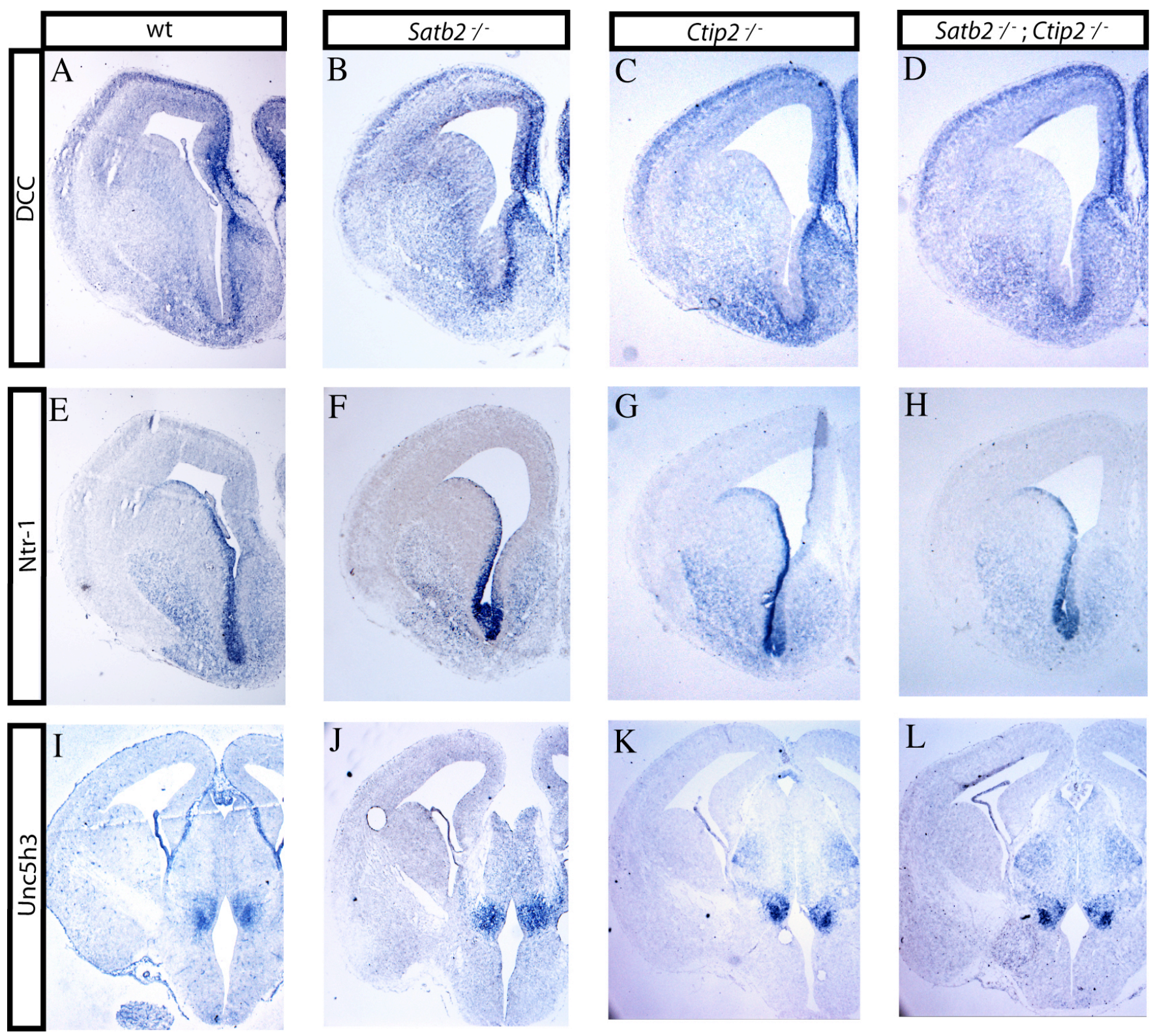

\subsubsection{Ctip2 directly binds to Unc5h3 promoter elements in vivo, and regulates} Unc5h3 expression in vitro

Our data thus far had shown that Ctip2 is repressing Unc5h3 expression, based on its restoration in Satb2 deficient neurons after the additional deletion of Ctip2 and on their non-overlapping expression patterns. We next wanted to examine whether the repression exerted by Ctip2 on Unc5h3 was direct. The initial database analysis showed that Unc5h3 promoter region has at least two putative Ctip2 binding sites (Fig. 24A). In order to test whether Ctip2 binds to Unc5h3 promoter in vivo, we 
isolated basal ganglia and cortices from neonatal wild type brains and performed chromatin immunoprecipitation (ChIP) analysis (in colabaration with Dr. Olga Britanova). These experiments showed that Ctip2 could indeed bind to the area ($60+100$ ) around the Unc5h3 initiation site in tissue isolated from basal ganglia (Fig. 24B). The absence of an analogous strong binding in cortical tissue could be due to the fact that the number of Ctip2 cells in the cortex is very low compared to the high number of Ctip2 expressing cells present in the basal ganglia. Furthermore, the above binding was shown to be specific for Ctip2, since upon the addition of non-specific IgG no such binding was observed. Moreover, we sought to investigate whether this binding of Ctip2 to Unc5h3 promoter area was also accompanied by the regulation of Unc5h3 expression. To test for this, we used HEK cells in which plasmids encoding the putative binding sites were transfected with or without the co-expression of full length Ctip2 and the amount of fluorescent protein induced was subsequently measured (luciferase assays). These experiments showed that upon Ctip2 addition there is an almost two fold decrease in the amount of luciferase protein produced for both putative binding sites tested (Fig. 24C). The above data confirm that Ctip2 is regulating Unc5h3 expression by showing that Ctip 2 can directly bind to Unc5h3 promoter putative binding sites in vivo and can repress Unc5h3 expression in vitro.

Fig. 24. Ctip2 binds $U n c 5 h 3$ promoter region in vivo and regulates $U n c 5 h 3$ expression in vitro. (A) Scheme showing the two putative Ctip2 binding sites (black boxes) in the Unc5h3 promoter area and the position of the primers used. (B) PCR for the $(-60+100)$ Unc5h3 promoter site, after ChIP assay with Ctip2 antibody and IgG, as a control. Strong binding can be seen just with Ctip2 (and not with $\operatorname{IgG}$ ) in tissue isolated from the basal ganglia (BG) of P0 wt brains. The GAPDH gene was also used as a control. (C) Two putative Ctip2 binding sites (E1, E2) were cloned into pC1-TK-luciferase vector and trasfected into HEK cells. When a plasmid expressing the full length CTIP2 cDNA was cotrasfected in the cells, there was an almost 2 fold decrease in the fluorescence ratio observed in both cases. All $\mathrm{p}$ values were $\leq 0,05$. (Brain material isolation and experimental design was done in collaboration with Dr. Olga Britanova; conductance of experiments and gel photos were taken by O.B., data analysis/interpretation was done by the author) 
A Unc5h3 promoter

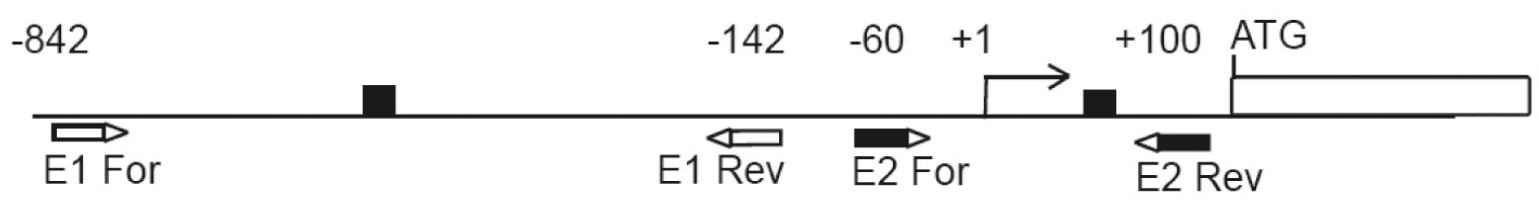

- CTIP2 binding site

B

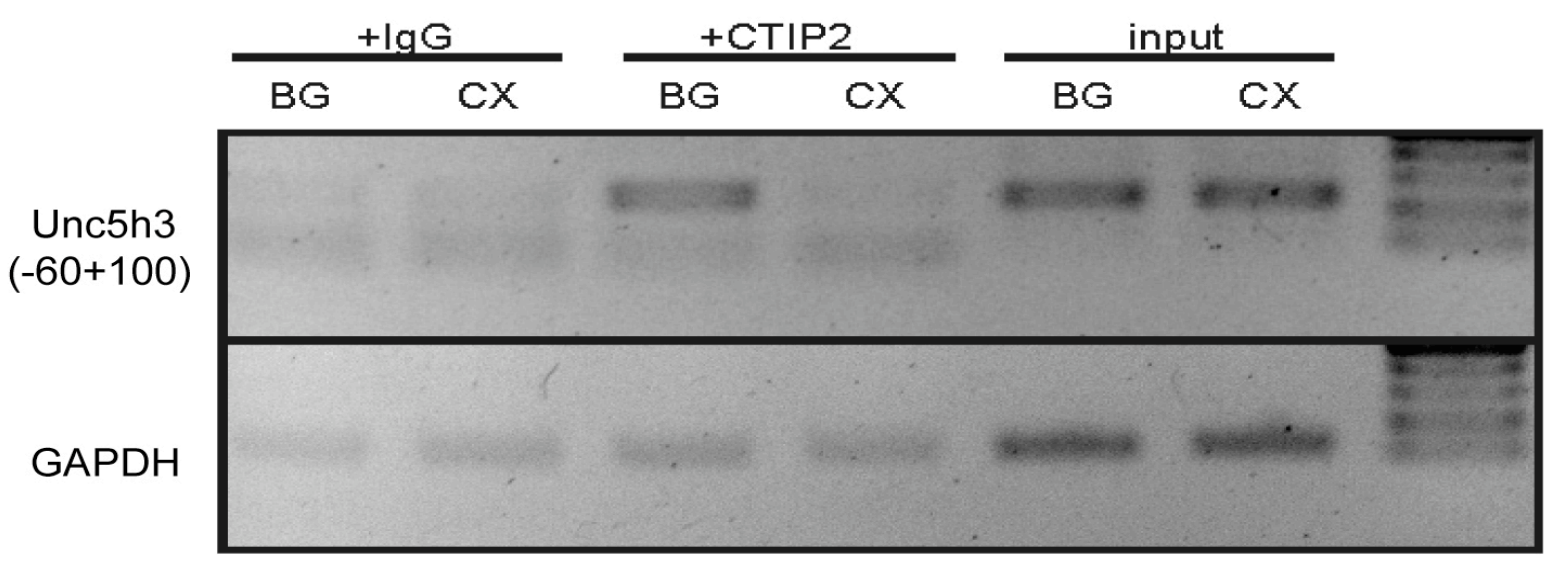

C

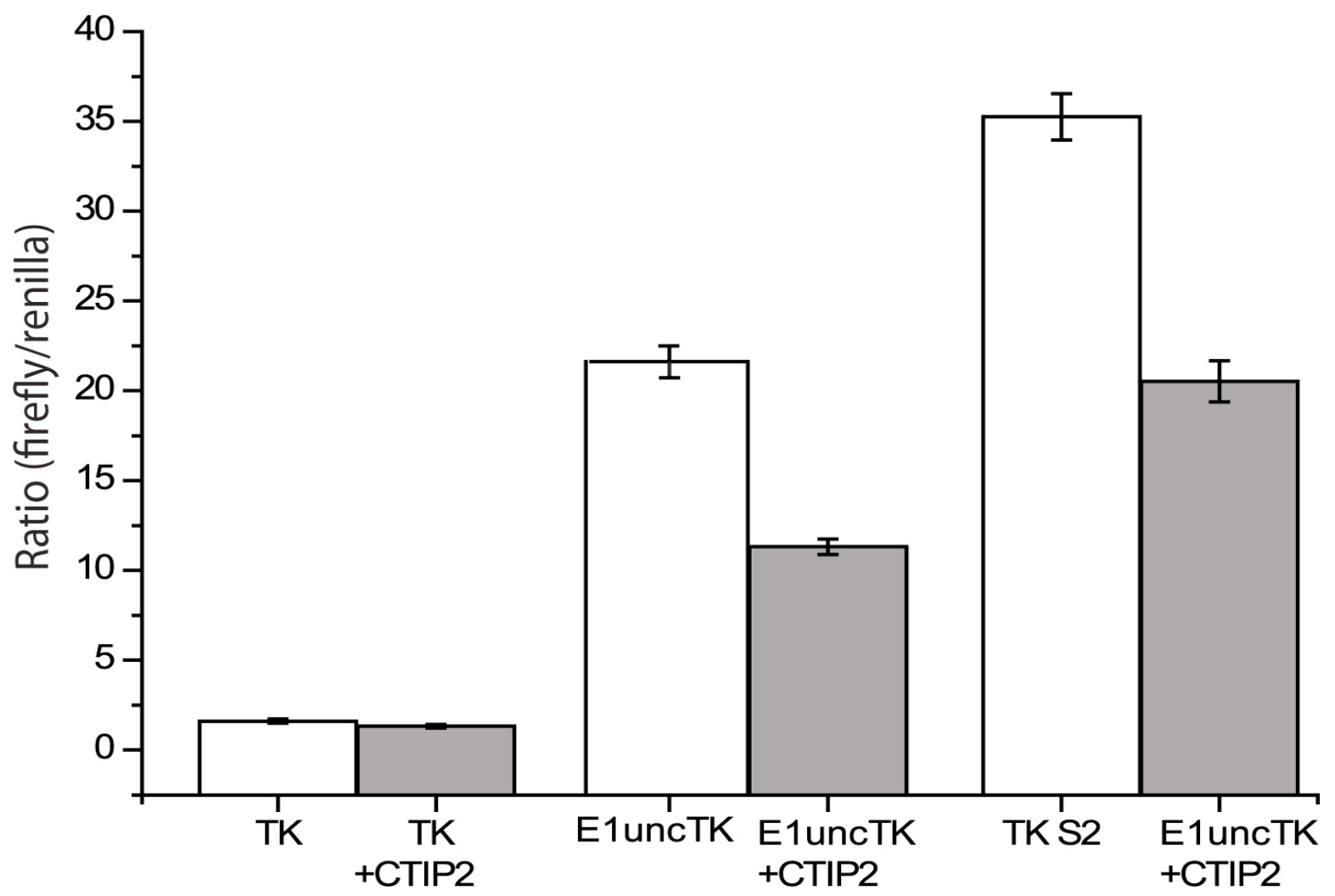




\subsection{Mechanisms delineating corticofugal projection identity}

\subsubsection{Patrial restoration of subcortical projections in the $\mathrm{Satb}^{-\alpha} ; \mathrm{FezI}^{--}$ compound mutants}

It has been reported that upon Fezl deletion there is an upregulation of Satb2 expression in the deeper cortical layers, a feature that might be responsible for giving these neurons a partial callosal identity (Chen, B. et al., 2008; Lodato, S. et al., 2011). In light of these results, we hypothesized that if we delete Satb2 in the Fezl mutant we might be able to remove the ectopic callosal identity from these deep layer neurons that upregulate Satb2, and therefore restore their normal subcortical identity along with aspects of their proper projections.

For this reason, $\mathrm{Fezl}^{--} ; \mathrm{Satb2}^{-/-}$compound mutants were generated and initially examined histologically by Nissl staining. As seen in Fig. 25C, in the $\mathrm{Fezl}^{-/}$which is lacking all subcerebral projections (Molyneaux et al., 2005) there is a robust decrease in the axonal bundles that transverse the internal capsule compared to the double heterozygote animals $\left(\mathrm{Fezl}^{-+} ; \mathrm{Satb2}^{+/}\right)$and the Satb2 mutants. However, after the additional deletion of Satb2 in the Fezl mutants $\left(\mathrm{Fezl}^{--} ; \mathrm{Satb2}^{--}\right)$, there was a noticeable increase in the white axonal bundles observed in the internal capsule (Fig. 25D, green arrows). Furthermore, hippocampal structures like the fimbria and the fornix that were atrophic or absent in the $\mathrm{Fezl}^{-/}$seemed to be, at least partially, restored in the $\mathrm{Fezl}^{-/} ; \mathrm{Satb2}^{-/}$brains (Fig. 25G and H, red and black arrows).

Fig. 25. Histological analysis of $\mathrm{Fez}^{/-} ; \mathrm{Satb}^{-/-}$compound mutants. Nissl staining on rostral and caudal sections of the four different genotypes indicated. In the Fezl mutants (C) the internal capsule axonal bundles (green arrow) are greatly reduced compared to the double heterozygote (A) and the Satb2 mutant (B) brains. In the compound mutants (D) however, the axonal bundles transversing the I.C. (green arrow) were markedly increased compared to the Fezl single mutant brains. In more caudal sections, the hippocampal structures, fimbria (black arrows) and fornix (red arrows) that were highly atrophic in the $\mathrm{Fezl}^{--}(\mathrm{G})$ are more evident in the $\mathrm{Fezl}^{-/} ; \mathrm{Satb}^{-/-}$compound mutants (H). 


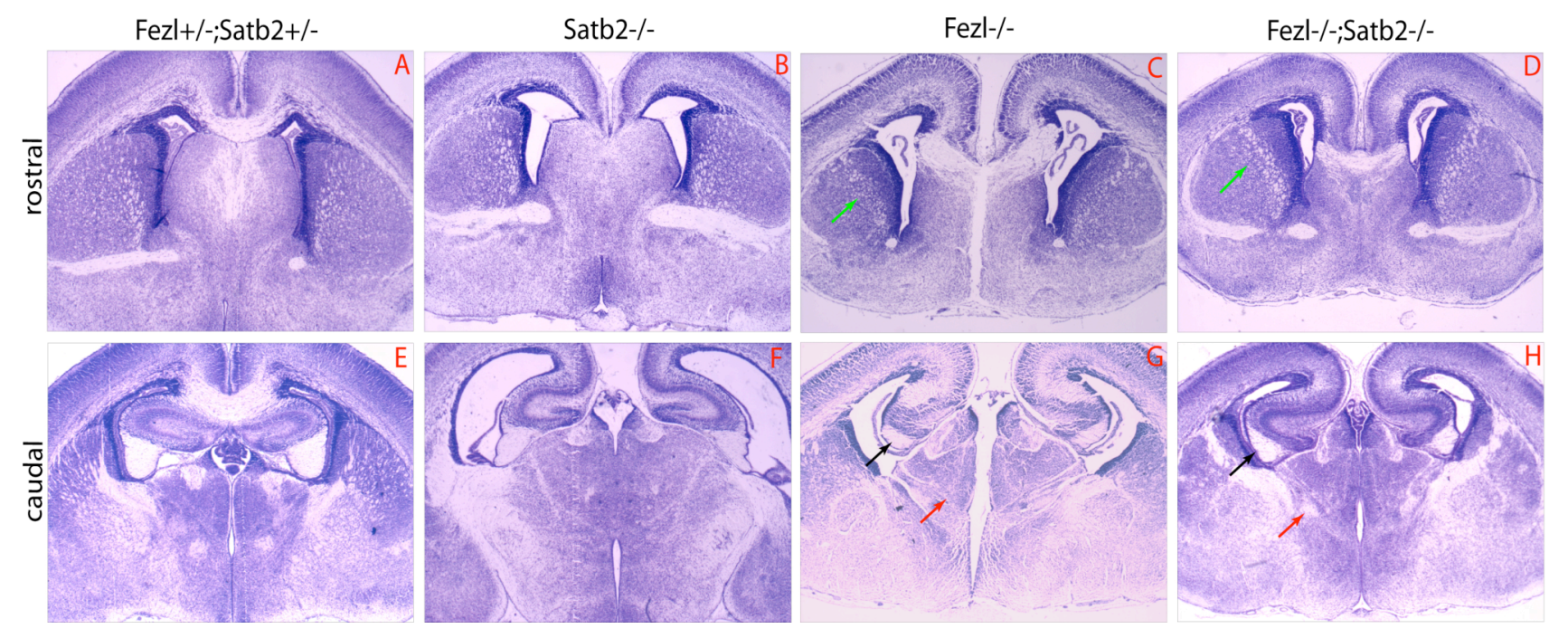

To further confirm the partial restoration of subcortical projections in the compound mutants and to trace the trajectories of the subcortically projecting neurons, DiI/A experiments were performed in collaboration with Srinivas Parthasarathy. Anterograde labeling of cortical neurons from the primary visual (V1) and the primary motor (M1) cortices using the lipophilic dyes DiA and DiI respectively showed a great reduction in the number of axons passing through the cerebral peduncle (C.P.) in the Fezl mutant brains from both the visual and the motor cortices. Hardly any labeling could be detected in either the dorsal (green) or ventral (red) part of the C.P. (magenta arrow). Interestingly, in the $\mathrm{Fezl}^{\gamma^{-}} ; \mathrm{Satb}^{-/-}$compound mutants there was a pronounced increase in the labeling of subcortical axons that transversed the C.P. originating from both the visual and the motor cortices (Fig. 26, magenta arrows), reinforcing the notion of the partial rescue of subcortical projections.

We next applied the same technique for axon visualization, only we sectioned the brains saggitally in order to better trace the axonal trajectories. The above labeling showed that there is a small number of axons that pass through the cerebral peduncle in the Fezl $\mathrm{F}^{--}$(Fig. 27B), however in the compound mutant the axonal bundle transversing the C.P. seemed to be considerably thicker (Fig. 27D, white arrow). In more lateral sections one can see that the CSMN axons in the wild type (Fig. 27E, white arrow) and in the Satb2 mutant form the pyramidal tract, which is completely missing in the Fezl mutant brains. It is noteworthy, that although in the compound mutant there are more axons passing through the C.P., compared to the Fezl single 
mutant (Fig. 27), they do not seem to project toward the spinal cord or form the pyramidal tract but instead project to arbitral targets, possibly to some pontine nuclei (Fig. 27F, white arrow). It is also interesting to note, that in the Satb2 mutants there is an unusual bundle of axons that is directed away from the A.C., to project caudally (Fig. 27C, white arrow). The origin of these axons and the cause for the above misrouting, are unknown.
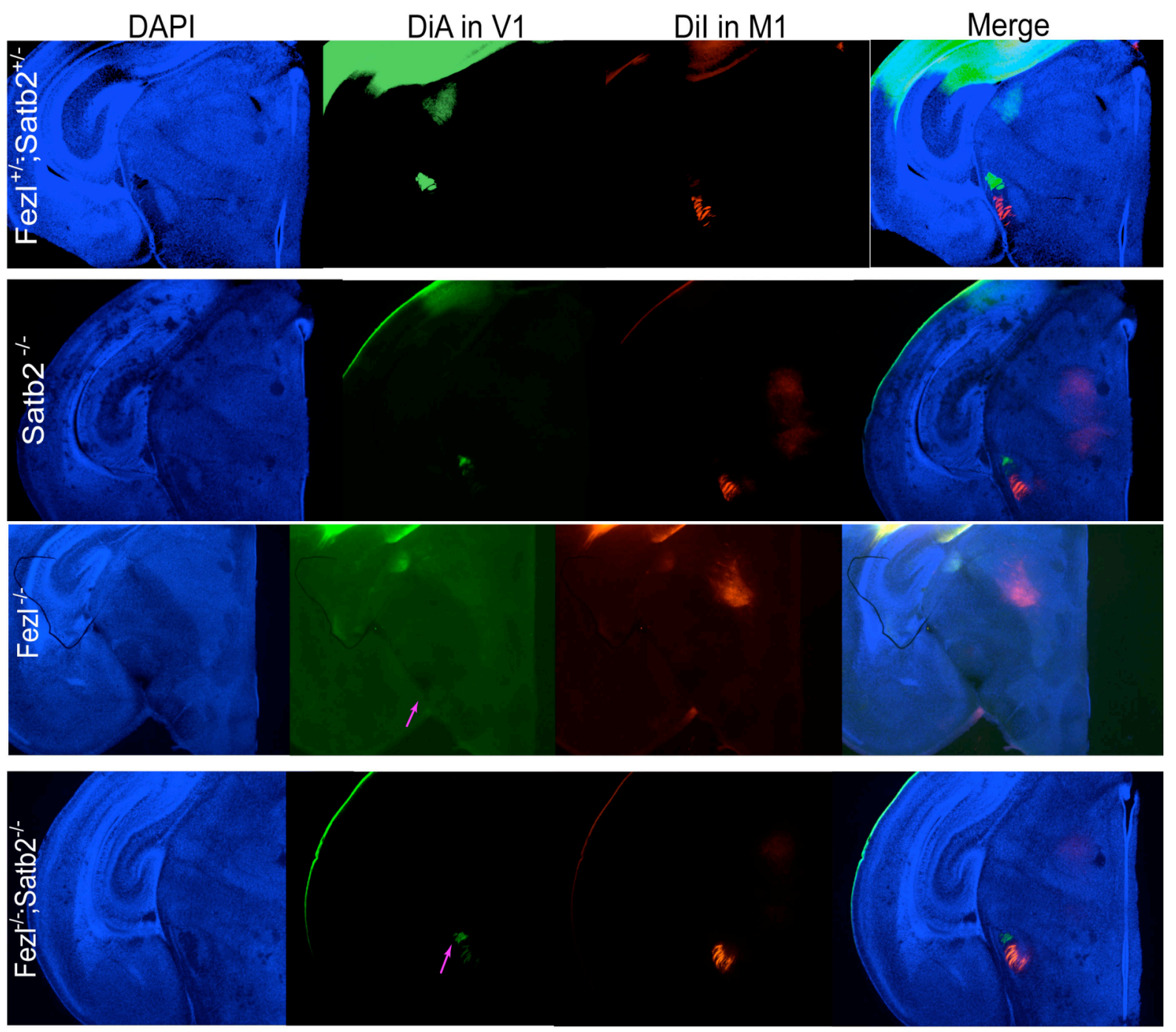

Fig. 26. Partial restoration of subcortical projections. Coronal sections of DiI (in primary motor cortex, M1) and DiA (in primary visual cortex, V1) labeled P0 brains of the four different genotypes indicated. In the $\mathrm{Fezl}^{-/}$there is hardly any labeling visible in the C.P. (magenta arrow), whereas in the $\mathrm{Fez}^{/-}$;Satb2 $2^{-/}$compound mutant there is a clearly evident labeling of axons transversing the C.P (magenta arrow) from both the M1 (ventral C.P., red) and V1 (dorsal C.P., green) cortices $(n \geq 2)$. (DiI placement was done in collaboration with Srinivas Parthasarathy; brain slicing and photo acquisition were performed by S.P.; brain collection/processing, experimental design and data analysis/interpretion were done by the author). 

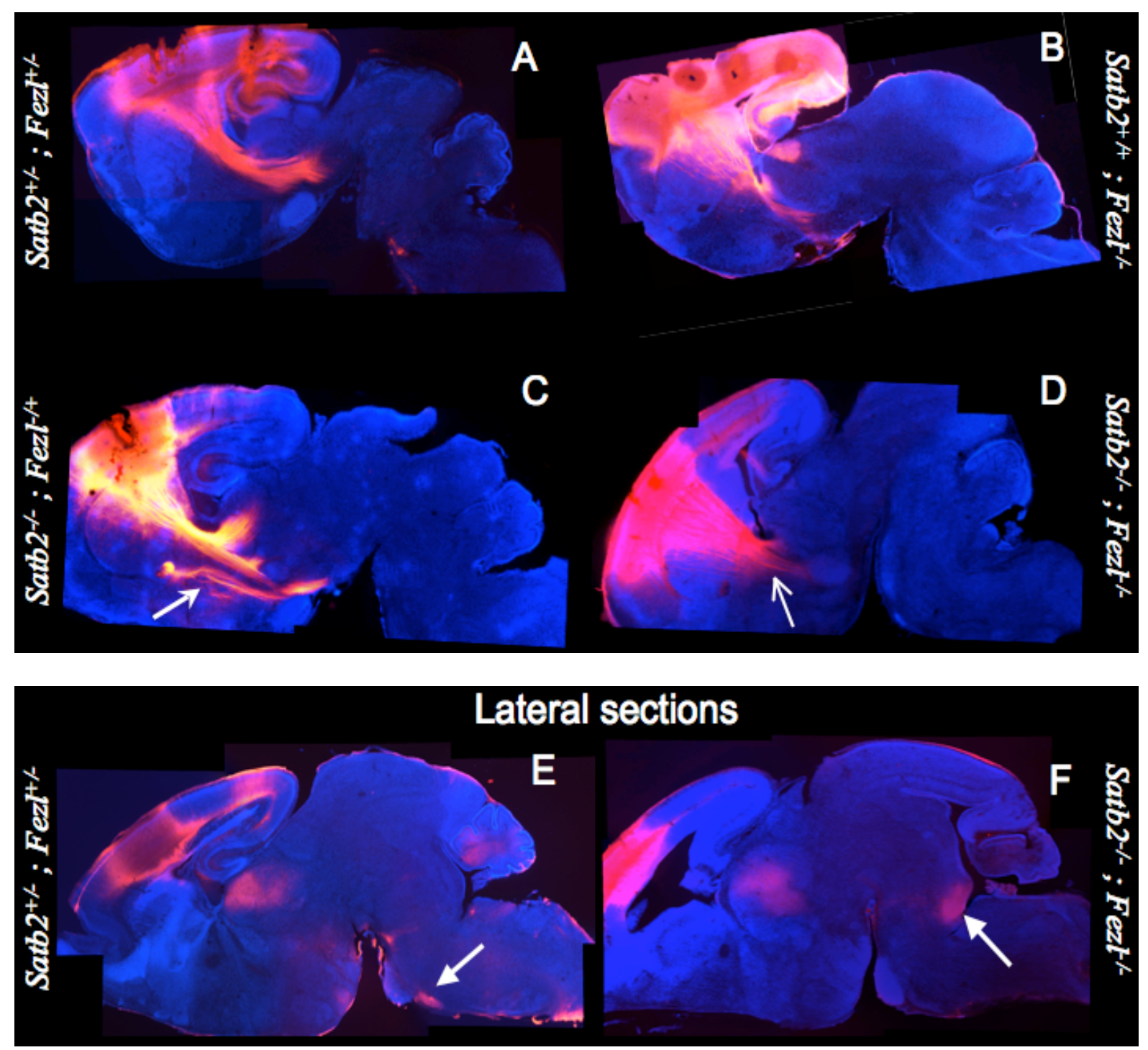

Fig. 27. Tracing of corticofugal connections; Anteriograde DiI lebeling from the motor cortex of P0 brains. The very limited number of axons passing through the C.P. in the $F e z l^{/-}(\mathrm{B})$ is increased in the case of the $\mathrm{Fezl}^{\gamma_{-}} ; \mathrm{Satb}^{-/-}$compound mutant (D, white arrow). In the latter, these C.P. transversing axons project somewhere in the pontine area (F, white arrow) and do not form the corticospinal tract like they do in the double heterozygote brain (E, white arrow). An unusual axonal bundle is misrouted, in the Satb2 $2^{-/}$, into projecting caudally to the A.C. (C, white arrow). (contributions as in Fig. 26).

In order to examine the origin of the neurons that project subcortically and are responsible for the partial rescue observed in the compound mutants, we decided to retrogradely label the cortical neurons that project to subcerebral targets. To this end, we placed DiI crystals in the C.P. of P0 wt, Fezl ${ }^{-/}, \mathrm{Satb2}^{-/-}$and $\mathrm{Fezl}^{\gamma_{-}} ; \mathrm{Satb2}^{-\alpha_{-}}$brains and examined the neurons that were back labeled in the cortex. These experiments showed that in the $\mathrm{Fezl}^{--}$there was very few cortical neurons labeled (Fig. 28), and 
their dendritic morphologies were severely altered with the apical dendrites mostly lacking terminal branches. In the Satb2 $2^{-/}$the neuronal labeling in the cortex except for layer V type I neurons, and similar to what is observed in the double heterozygote brains (A, A'), also included cells found in the upper cortical layers (Fig. 28B', white arrow). In the compound mutant a considerable number of neurons were efficiently back labeled, most of which seemed to reside in the deeper cortical layers. It is not exactly clear what kind of cells represented the labeled population, however these cells, as shown in Fig. 27 do not project all the way to form the corticospinal tract.

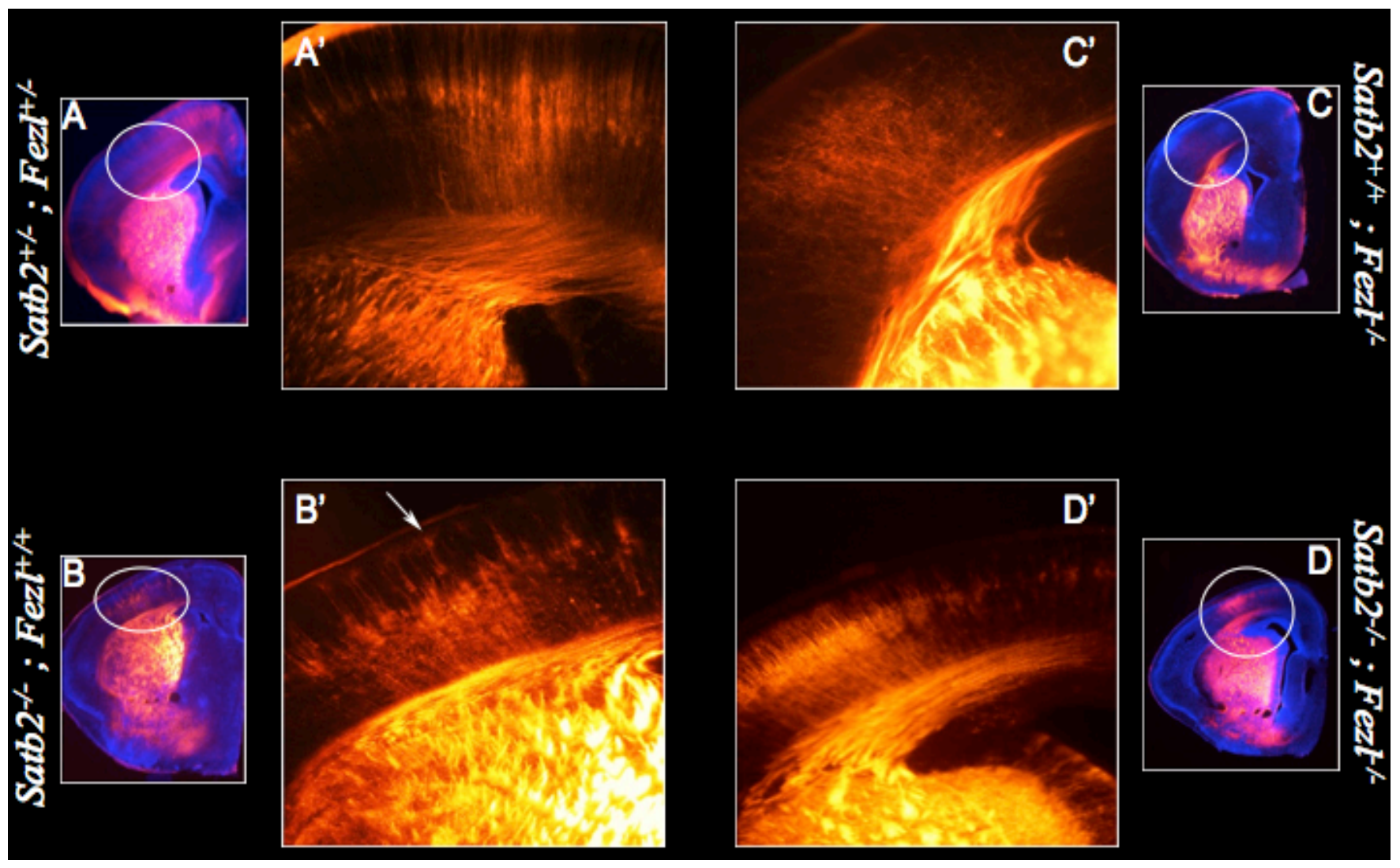

Fig. 28. Retrograde cortical labeling from the cerebral peduncle. DiI placement in the C.P. of P0 animals resulted in the backlabeling of cortical cells that sent axons through the C.P. to subcerebral targets. (A') shows the nicely labeled layer V neurons that project to subcerebral targets in double heterozygote brains and (B') shows the same labeling in the Satb2 $2^{-/}$with some upper layer neurons being labeled as well (white arrow). In (C') the back labeling in the Fez/ $\mathrm{I}^{--}$is very limited and it looks like the neuronal dendrites labeled cells are rather disorganized/disoriented. Panel (D') shows that there are a considerable number of cortical neurons being back labeled in the $\mathrm{Fezl}^{+/} ; \mathrm{Satb2}^{+/}$, most of which seem to reside in the DL. (A', B', C' and D' are higher magnification pictures of the circled areas in A, $\mathrm{B}, \mathrm{C}$, and D respectively). (contributions as in Fig. 26) 


\subsubsection{Present and grossly normal the corticothalamic projections in all three different mutants $\left(\mathrm{Satb}^{-/}, \mathrm{Fez} \Gamma^{--}\right.$and $\left.\mathrm{Satb}^{--} ; \mathrm{Fez}^{--}\right)$examined}

We next wanted to examine whether the corticothalamic projections are intact in both the $\mathrm{Fezl}^{-/}$and the $\mathrm{Fezl}^{-/} ; \mathrm{Satb2}^{-/}$. In the Satb2 $2^{-/}$it has been previously reported that the thalamocortical axons project normally through the internal capsule to the cortex. However, similarly to the corticofugal connections, the TCAs projection pathway in the Satb2 $2^{-/}$was sifted to more posterior levels compared to the wt brains (Britanova et al., 2008). DiA and DiI placement in the V1 and M1 cortices respectively, showed that in all three mutant genotypes $\left(\mathrm{Fezl}^{\gamma_{-}^{-}} ; \mathrm{Satb}^{-/}, \mathrm{Fez}^{\gamma_{-}}\right.$and $S a t b 2^{-/}$) the cortical neurons send axons to the appropriate areas in the thalamus (Fig. 29). DiA placement in the visual cortex resulted in labeling of the lateral geniculate nucleus (LGN) of the thalamus (green), whereas DiI placement in the motor cortex resulted in labeling of the ventrolateral thalamic nucleus (red) in all genotypes examined. Although this type of assay does not allow for accurate quantitative analysis, the above experiments showed that all the major aspects of corticothalamic and thalamocortical projections are present and grossly normal.

Fig. 29. Examination of corticothalamic projections. DiA was placed in the primary visual (V1) and DiI in the primary motor (M1) cortices of wt, $\mathrm{Fezl}^{-/}, \mathrm{Satb2}^{-/-}$and $\mathrm{Fezl}^{-/} ; \mathrm{Satb2}^{-/-} \mathrm{P} 0$ brains. In all four genotypes, neurons from the visual cortex were sending axons to the lateral geniculate nucleus of the thalamus (green) whereas neurons for the motor cortex were sending axons to the ventrolateral thalamic nucleus (red). Although detailed quantitative analysis was not performed, the trajectories of the corticothalamic and thalamocortical projections appeared grossly normal. (contributions as in Fig. 26) 

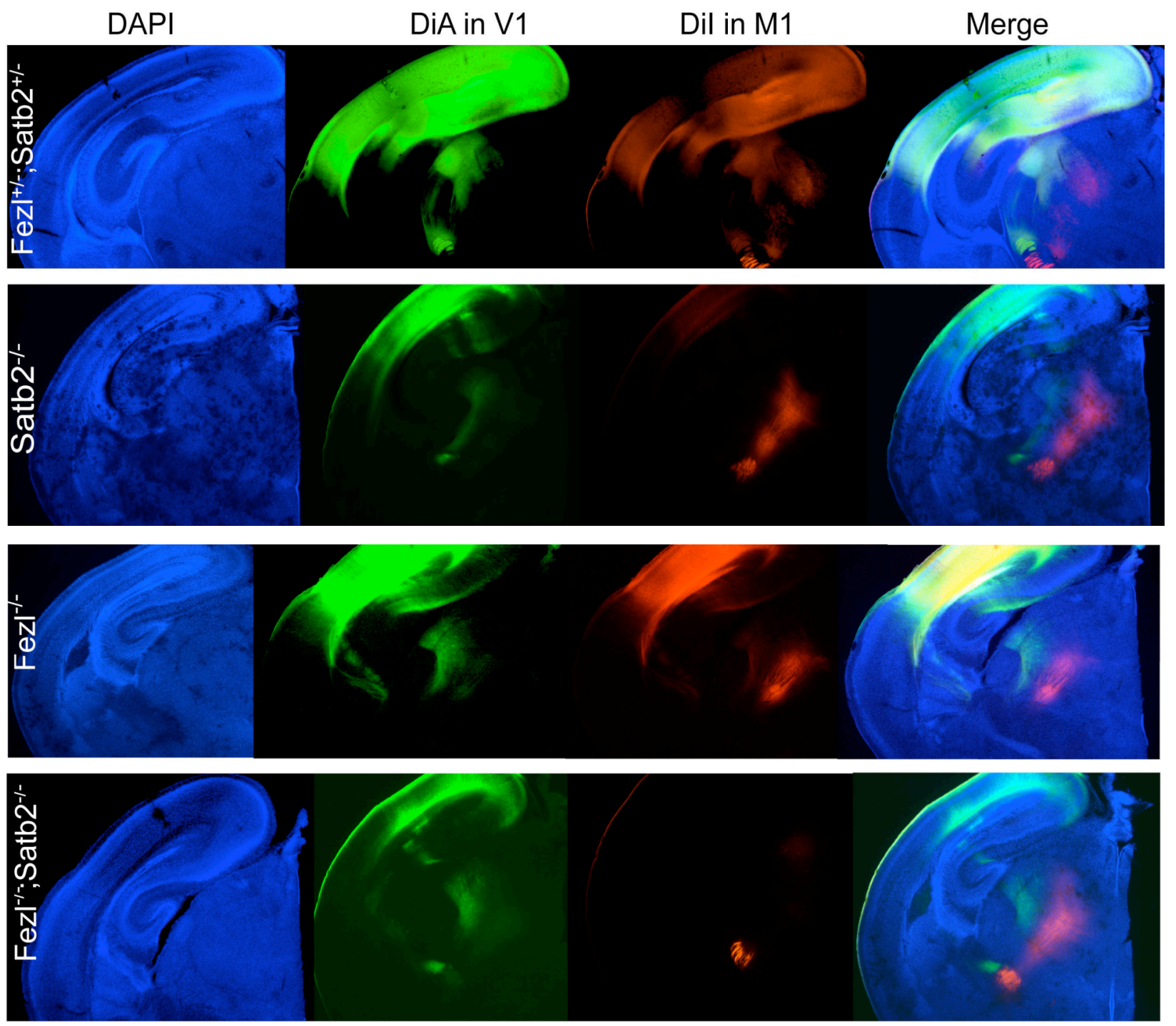

3.2.3 Subcortical projection neuronal identity does not seem to be fully restored in the $\mathrm{Satb2} 2^{-/-} ; \mathrm{Fez} \Gamma^{-}$

Since the subcerebral projections, that were almost completely missing from the $\mathrm{Fezl}^{-/}$were partially restored in the $\mathrm{Satb2}^{-/} ; \mathrm{Fezl}^{-/}$, we wanted to examine in more detail the nature of the above restoration. In the Fezl single mutant there is a complete absence of the typical type I layer V neurons that show high Ctip2 expression, whereas in layer VI, which is slightly expanded, the Ctip2 expression is maintained (Molyneaux et al., 2005). The reported expansion of Satb2 expression in the deeper cortical layers of the $\mathrm{Fez}^{-/}$could possibly account for the absence of Ctip2 from these layers, since Satb2 is known to repress Ctip2 expression (Britanova et al., 2008; 
Alcamo et al., 2008).

If that were the case, then upon the additional deletion of Satb2 from the Fezl mutant cortex there would be a restoration of Ctip2 expression in the deep cortical layers. To test this possibility, IHC for Ctip2 expression was performed in P0 brains. The above experiments confirmed the lack of Ctip2 expression in layer $\mathrm{V}$ of the $\mathrm{Fezl}^{-/}$ (Fig. 30C) and showed that upon additional deletion of Satb2 there seems to be a dispersed expression of Ctip2 throughout the whole cortex of the $\mathrm{Fezl}^{T^{-}} ; \mathrm{Satb}^{-/}$brains (Fig. 30D). A closer examination of the mutant cortices indicates that the thinner cortex that has previously been reported for the Satb2 $2^{-/}$(Britanova et al., 2008; Alcamo et al., 2008) seems to regain almost normal size in the compound mutants (Fig. 30C' and D'). The Ctip2 ectopic expression in the upper cortical layers is present in the compound mutant, similar to what is observed in the $S a t b 2^{-/}$, whereas in the deeper layers there seems to be cells that regain relatively high Ctip2 expression. However, these cells do not reside exclusively in layer V but look rather dispersed throughout the deeper cortical layers (Fig. 30D', white arrow). Additionally, it is not absolutely clear whether these cells are indeed type I layer $\mathrm{V}$ neurons or other types of deep layer Ctip2 expressing neurons. Given that their projection pattern is also not entirely restored (even though they project subcerebrally, they do not form the CST like they normally should, Fig. 27), it is evident that these cells do not fully regain their proper neuronal identity.

In order to further examine the proper lamination of the $\mathrm{Fezl}^{-\alpha} ; \mathrm{Satb2}^{-/}$cortex we decided to check for the expression patterns of other layer specific markers as well. Brn1 and 2 have been shown to play a cell autonomous role in the regulation of the neurogenesis of upper layer neurons (Sugitani et al., 2002). It has been reported that in the $S a t b 2^{--}$, although the total number of Brn1/2 positive cells was comparable to that of wt animals there was an accumulation of these cells in the IZ of the mutant cortex (Britanova et al., 2008). Examination of Brn2 expression in the three different mutant brains with IHC at P0, revealed that the increased number of Brn2-positive cells in the IZ observed in the Satb2 $2^{-/}$(Fig. 30G, dashed lines) is also evident in the double mutant $\mathrm{Fezl}^{\gamma^{-}} ; \mathrm{Satb2}^{-/}$(Fig. 30H, dashed lines). At the same time the Brn2 positive cells appeared significantly reduced in the upper cortical layers of the $S a t b 2^{-/-}$ compared to the control brains, and this reduction is also maintained in the compound mutant (Fig. 30E, G and H, white arrows). The above result indicates that the migration defects of upper layer cortical neurons that characterize the Satb2 $2^{-/}$ 
(Britanova et al., 2008) are most likely present in the $\mathrm{Fezl}^{\gamma^{-}} ; \mathrm{Satb2}^{-\alpha_{-}}$as well.
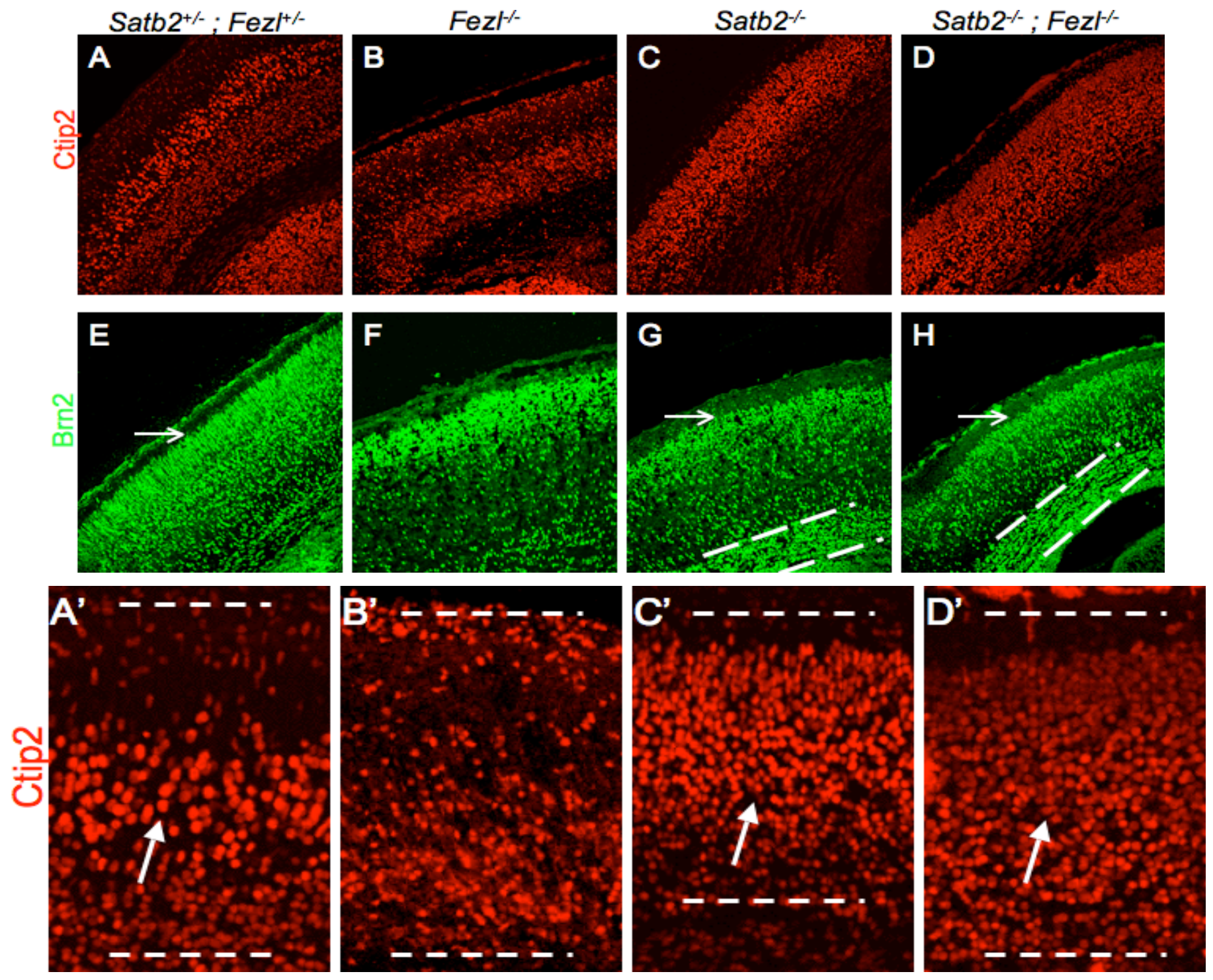

Fig. 30. Examination of neuronal specificity/proper lamination in the compound mutant cortex.

The expression of Ctip2 and Brn2 was examined by IHC in the four different genotypes indicated. A, B, C and D are coronal sections at the level of the somatosensory cortex of $\mathrm{Fezl}^{+/} ; \mathrm{Satb2}^{+/}, \mathrm{Fezl}^{/-}$, $\mathrm{Satb2}^{-/}$and $\mathrm{Fezl}^{-/} ; \mathrm{Satb}^{-/}$brains respectively, immunostained for Ctip2. E, F, G and $\mathrm{H}$ are sections of the above genotypes stained for Brn2 where it can be seen that Brn2 expression is reduced in the upper layers of the $\mathrm{Satb2}^{-/}$(G, white arrow) and the $\mathrm{FezT}^{-\sim} ; \mathrm{Satb2}^{-/}$mutants (H, white arrow) compared to its expression in the double heterozygote brain ( $\mathrm{E}$, white arrow). At the same time there is an accumulation of Brn2 positive cells in the IZ of the $\mathrm{Satb}^{-/}$(G, dashed lines) and $\mathrm{Fezl}^{-\kappa} ; \mathrm{Satb}^{-/}$( $\mathrm{H}$, dashed lines) indicative of similar migration defects in the two mutants. A', B', C' and D' are blowups of A, B, C and D respectively, where the cortical distribution of Ctip2 positive cells could be examined in more detail. The dashed lines show the thickness of the cerebral cortex which is markedly reduced in the case of the $\mathrm{Satb2}^{-/}$but appears rather normal in the $\mathrm{Fezl}^{-} ; \mathrm{Satb}^{-/}$. The distribution of Ctip2 
positive cells is shown to be irregular throughout the deep layers of the $\mathrm{Fezl}^{\digamma_{-}} ; \mathrm{Satb2}^{-/-}$(D', arrow) and not properly confined in layer V like in the $\mathrm{Fezl}^{+/} ; \mathrm{Satb2}^{+/-}$(A', arrow) and Satb2 ${ }^{-/}$(C', arrow). Although it is not absolutely clear that these cells are indeed the high Ctip2 expressing layer V neurons, it is evident (see also Fig. 27 and 28) that they do not regain all aspects of their proper neuronal identity.

We next wanted to examine the identity of the neurons that project to subcerebral targets in the $\mathrm{Fezl}^{--} ; \mathrm{Satb2}^{-/}$and are responsible for the partial rescue. For this reason, sections of $\mathrm{Satb2}^{-/}$and $\mathrm{Fezl}^{--} ; \mathrm{Satb2}^{-/}$double mutant P0 brains, that had previously been labeled with DiI at the cerebral peduncle, were stained for Ctip2. As seen in Fig. 31, the cells that are back labeled in the cortex reside in the deep layers (like they normally should) but there is also labeling of upper layer cortical neurons in both the Satb2 $2^{-/}$(Fig. 31A, white arrow) and the Fezl ${ }^{-\alpha} ; \mathrm{Satb2}^{-/-}$(Fig. 31D, white arrow) mutants. These upper layer cells that are labeled seem to be Ctip2 expressing neurons (Fig. 31C and F).

Furthermore, if one looks at the morphology of the back-labeled cells in the compound mutants, it becomes apparent that these cells have abnormal dendritic branches, similar to what is observed in the $\mathrm{Fezl}^{-/}$(see also Fig. 28C'). Additionally, the apical dendrites of the labeled cells in the $\mathrm{Fezl}^{-/} ; \mathrm{Satb2}^{-/}$do not extend their branches into layer I as they do in the Satb2 $2^{-/}$(Fig. 31A and D, red arrows), which is typical for layer $\mathrm{V}$ subcortically projecting neurons, but terminate in deeper layers and appear disorganized. This is also indicative of the fact that the cells responsible for the partial rescue of the subcerebral projections have not really regained their proper identity.

In our effort to examine this further, similar sections of double heterozygote $\left(\mathrm{Fezl}^{+/-} ; \mathrm{Satb2}^{+/-}\right)$and compound mutant $\left(\mathrm{Fezl}^{--} ; \mathrm{Satb2}^{-/}\right)$brains were stained for FNP7 , an antibody which is raised against the non-phosphorylated form of the medium sized neurofilament protein. It has been reported that FNP-7 is a marker for subcortically projecting pyramidal neurons and that it labels exclusively these cells within layer $\mathrm{V}$ and not the ones that form corticocortical connections (Voelker et al., 2004). Upon immunostaining with the above antibody, a lack of immunoreactivity in the deep layers of the $\mathrm{Fezl}^{-/} ; \mathrm{Satb}^{-/-}$was observed, especially in the lateral cortex (Fig. 32A and A', arrow). Contrary to the compound mutant, there was a uniform staining with FNP-7 throughout the whole medial to lateral axis of the $\mathrm{Fezl}^{+/} ; \mathrm{Satb2}^{+/-}$ 
cortex (Fig. 32B and B', arrow). The above result is a further indication that the specification of the deep cortical pyramidal cells, especially the type I layer $\mathrm{V}$ subcortically projecting neurons, is not proper in the compound mutants.

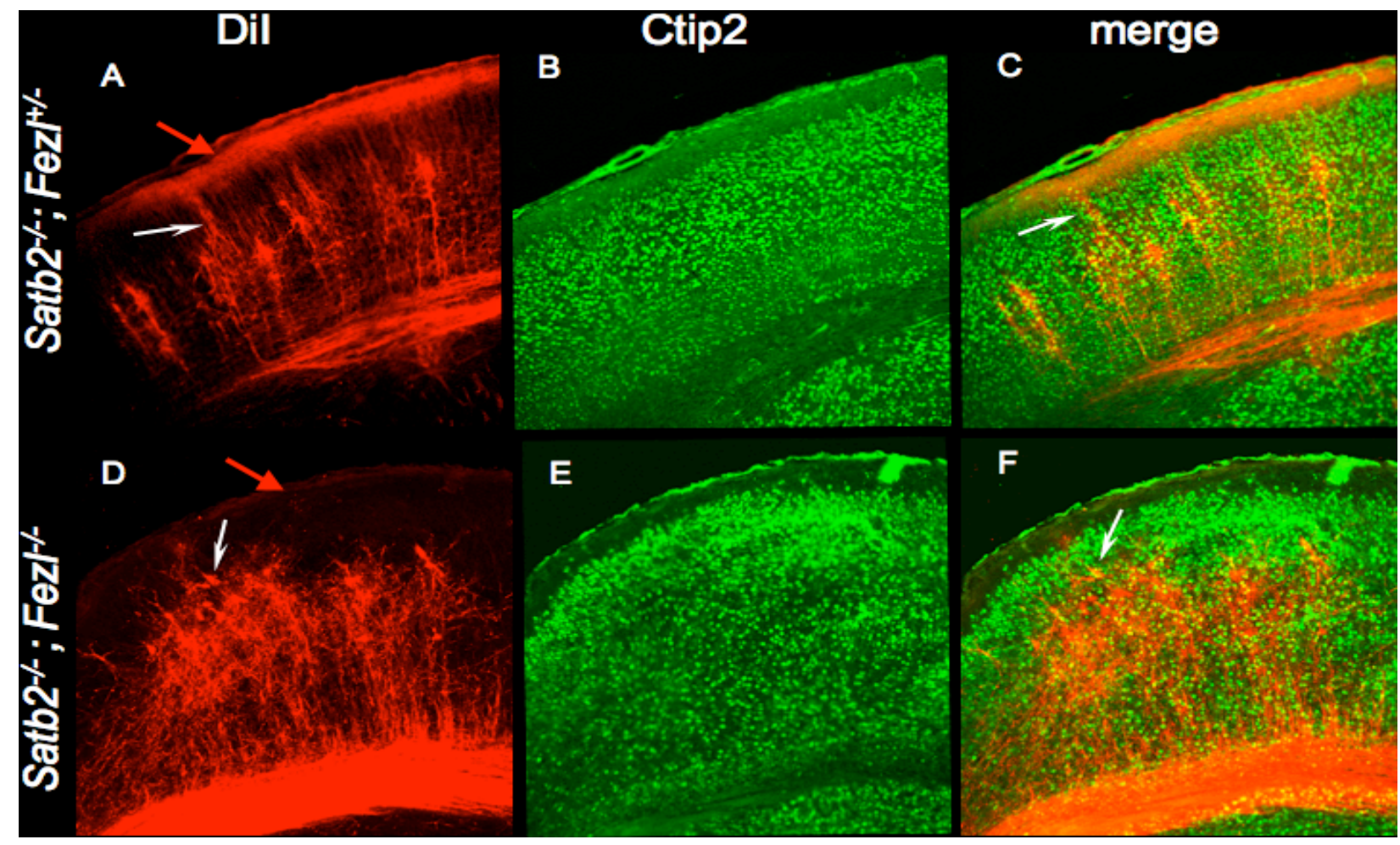

Fig. 31. Identification of subcortically projecting neurons in the $\mathrm{FezI}^{/-} ; \mathrm{Satb2}^{-/-}$. DiI back-labeling of cortical cells from the cerebral peduncle of Satb2 mutant (A, B, C) and compound mutant (D, E, F) P0 brains. In both mutants although most of the subcortically projecting neurons reside in the deep layers (mainly V), there are also labeled cells that are found in the upper cortical layers (white arrows in A, C, D and F). Most of the back-labeled neurons seem to also express Ctip2 (B, C and E, F). It is also noteworthy that the neurons of the $\mathrm{Fezl}^{-/} ; \mathrm{Satb2}^{-/}$have disorganized dendrites (seen as a messy fiber net in D) and do not extend an apical branch all the way up to layer I, like they do in the Fezl ${ }^{+/-}$ ;Satb2 $2^{-/}$(red arrows in A and D). (DiI placement was done in collaboration with S.P.; brains were sliced by SP.; brain collection/processing, IHC, picture acquisition, experimental design and data interpretation/analysis were performed by the author).

Except for the expression of the above subcortical specific neuronal marker (FNP-7), the expression of another molecule that primarily marks callosally projecting neurons was also examined. The LIM-only protein 4 (Lmo4) has been reported to label primarily $\mathrm{CPN}$ at their late differentiation stage (postnatal stages). Since the $\mathrm{Satb}^{-/}$and the $\mathrm{Fezl}^{\gamma^{-}} ; \mathrm{Satb}^{-/}$compound mutant embryos die perinatally, 

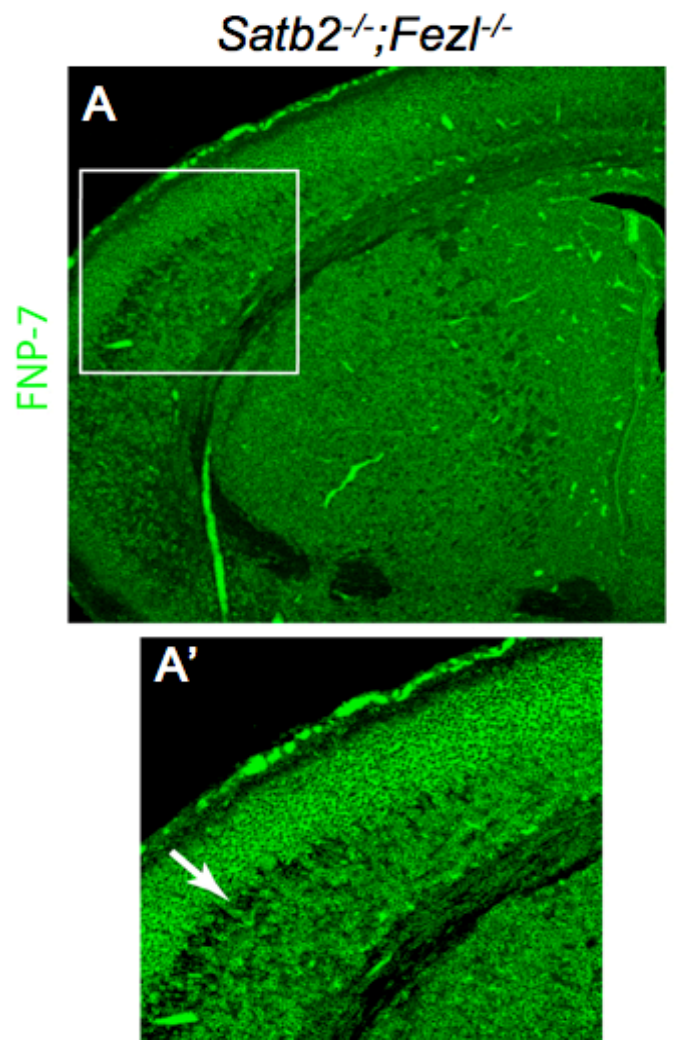

Satb2 $2^{+/ ;} ; \mathrm{Fez}^{+/-}$
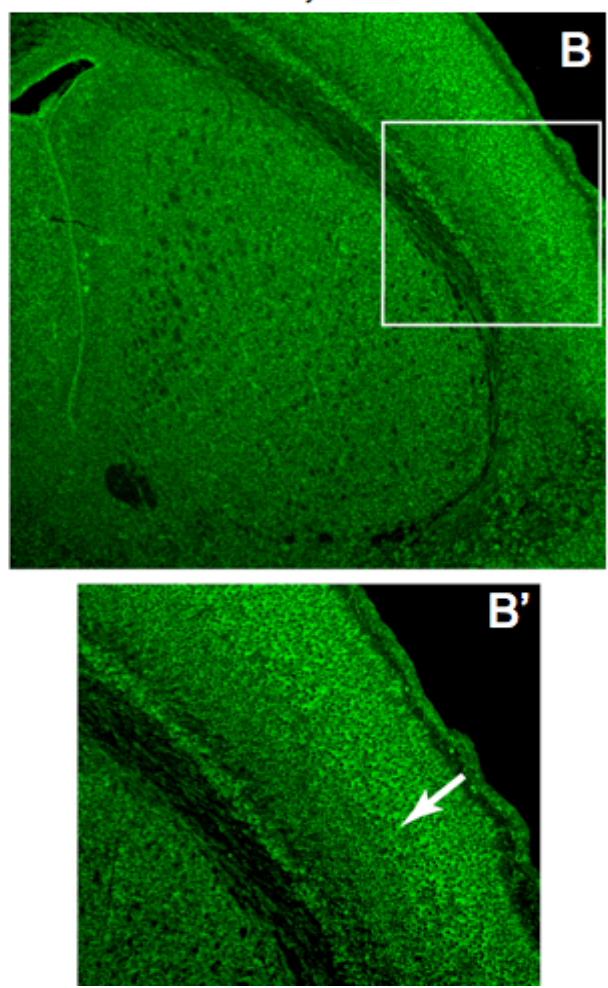

Fig. 32. Subcortically projecting neuron specific staining. Staining for FNP-7 in $\mathrm{Fez}^{\gamma^{-}} ; \mathrm{Satb}^{-/}$and $\mathrm{Fezl}^{+/-} ; \mathrm{Satb2}^{+/-}$coronal sections of P0 brains. FNP-7 has been shown to specifically label subcortically projecting neurons within layer $\mathrm{V}$ and not the neurons that project to the contralateral cortex. In the $\mathrm{Fezl}^{/-} ; \mathrm{Satb2}^{-/}$the immunoreactivity for FNP-7 is not present in parts of layer V, especially in the lateral cortex where layer $\mathrm{V}$ does not seem to be stained at all (A and A', white arrow). In contrast to the compound mutant, in the $\mathrm{Fezl}^{+-} ; \mathrm{Satb2}^{+/-}$brains the labeling for FNP-7 in the subcortically projecting neurons of layer $\mathrm{V}$ is uniform throughout the whole medial to lateral axis (B and B', white arrow).

the latest stage we could test for, was P0. At this stage the CPN are still in their middifferentiation stage and Lmo4 has become partially excluded from the subcerebral projection neuron population (Azim, A. et al., 2009). In terms of its rostrocaudal expression pattern, Lmo4 shows already by P0 a "gap" in its expression, in an area that is identified, by the expression of EphrinA5, as the somatosensory cortex (Bulchand, S. et al., 2003). In Fig. 33, where coronal sections of the somatosensory cortex are depicted, it is evident that in the $\mathrm{Satb}^{-/}$there is an obvious upregulation of Lmo4 in the upper layers of the somatosensory and cingulate cortices with a possible reduction in the deeper layers, although it is hard to discern due to the considerable reduction in the cortical thickness (Fig. 33C). Normally, the expression of Lmo4 is 
missing from the upper layers of the somatosensoty cortex, which is not the case for other more rostral or caudal cortical areas, while being present in a subpopulation of deep layer neurons at P0 (Fig. 33B). In the Fezt ${ }^{-/}$, Lmo4 is almost completely lost from the somatosensory cortex, while maintained in the cingulate cortex (Fig. 33E). Upon deletion of both Satb2 and Fezl the expression of Lmo4 is still persistent in the upper layers of the somatosensory cortex and seems to be, at least partially restored, in the deeper cortical layers (Fig. 33D).

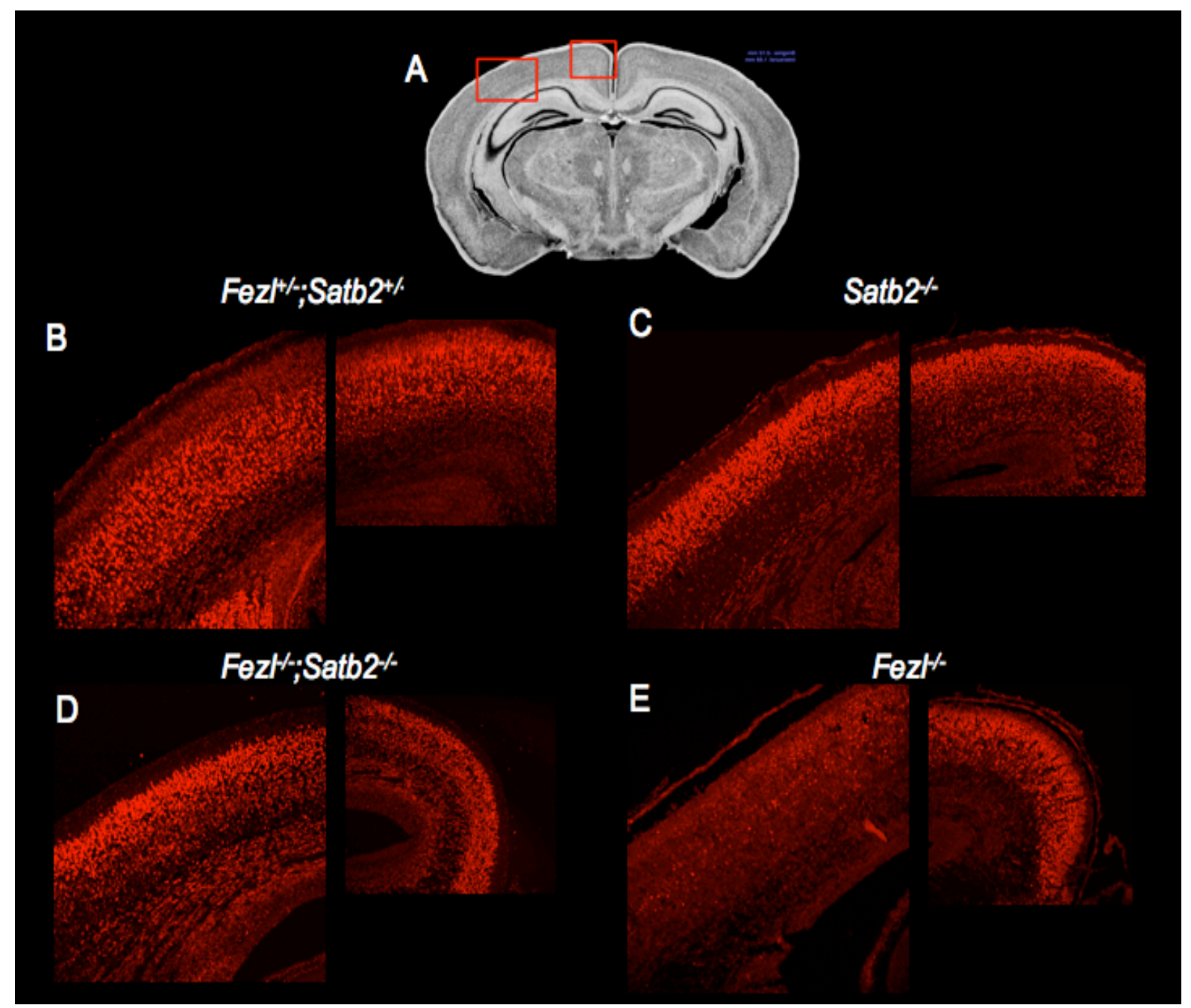

Fig. 33. Staining for Lmo4 at P0 (indermediate differentiation CPNs). (B) In the wt brains there is normally a "gap" in Lmo4 expression in the UL of the somatosensory cortex while this is not the case for the cingulate and for other, more rostral or caudal, parts of the cortex. In the Fez $\digamma^{--}(\mathrm{E})$ there is an almost complete lost of Lmo4 immunoreactivity from all layers of the somatosensory cortex, while in $\mathrm{Satb}^{-/}$there is an upregulation of Lmo4 in the UL (C). In the compound mutants the ectopic upregulation of Lmo4 in the upper cortical layers is maintained (similar to Satb2 ${ }^{-/}$), while at least part of the deep layer expression seems to be also restored (D). (A) shows a scheme of the parts of the somatosensory cortex (big red box) and cingulate cortex (small red box) depicted in the pictures. 


\section{DisCUSSION}

\subsection{Regulation of callosal projections in the mouse forebrain}

\subsubsection{Callosal neuron identity downstream of Satb2}

It has been clearly shown that Satb2 plays an important role in defining callosal neuron identity in the cortex (Alcamo et al., 2008; Britanova et al., 2008). One way by which Satb2 is acting in order to confer such identity, is through repressing the expression of Ctip2 in callosal projection neurons via the direct binding on the Ctip2 promoter. Ctip2, on the other hand, is a transcription factor robustly expressed in subcortical projecting neurons of layers V and VI with the highest expression in the type I layer $\mathrm{V}$ neurons that project axons subcerebrally to form the CST and to innervate the spinal cord (Arlotta et al., 2005). The deletion of Satb2, leads to an ectopic upregulation of Ctip2 in callosal projection neurons due to the loss of Satb2 repression on Ctip2. The result is that the above neurons instead of projecting to the contralateral side through the C.C. misproject to subcortical targets via either the A.C. or the internal capsule (Alcamo et al., 2008; Britanova et al., 2008; Gord Fishell and Carina Hanashima, 2008).

\subsubsection{The control of callosal specification}

From the above studies it becomes evident that Ctip2 is largely responsible for confining, postmitotically, a subcortical neuron identity. Given that the misexpression of Ctip2 in the Satb2 deficient cells is not as high as in normal layer V CSMN cells, it is rather expected that these neurons would, most likely, never make it to the spinal cord. Although not directly addressed, due to the perinatal lethality of the Satb2 ${ }^{-/}$ embryos, it is clear that, if not all, at least a considerable number of the ectopically Ctip2 expressing UL neurons misproject either to aberrant targets through the A.C. (Fig. 27C) or make various subcortical projections via the internal capsule and C.P. (Britanova et al., 2008) but not to the spinal cord (Alcamo et al., 2008). It is possible that, except for the ectopic upregulation of Ctip2 expression, the laminar position of these Satb2 deficient cells also plays a role in the type of misprojections that they will 
form. Therefore, most of the UL Ctip2+/Satb2- cells in the Satb2 $2^{-/}$do not fully acquire a normal subcortical identity.

To extent this analysis, the current study reveals some additional modes of action by which Satb2 confers a callosal neuron specification. In our effort to address the question whether the presence of Ctip2 alone, is sufficient to drive the UL neurons of the $S a t b 2^{-/-}$to project subcortically, we generated Ctip2 $2^{-/} ; S a t b 2^{-/-}$compound mutants in which we could examine the projections of these neurons. At first glance, the absence of an obvious restoration of the C.C. in the Ctip2 $2^{-/} ; \mathrm{Satb}^{-/}$points to the fact that Ctip2 is not the only and/or the main factor that makes Satb2 deficient cells project subcortically. Therefore, it is clear that the absence of Ctip2 alone is not sufficient to restore the callosal identity of the Satb2- cells. Similarly, the presence of Ctip2 is not necessary for a neuron to acquire an ectopic subcortical identity, since overexpression of Fezl in the UL of the $\mathrm{Fezl}^{-/-}$mice results in these cells sending subcortical instead of callosal projections without, however, inducing Ctip2 expression or having any effect on the expression of Satb2 (Chen, B. et al. 2008). All of the above indicate that except for the mere fact of the types of transcription factors expressed by a neuron, there are also additional parameters that contribute to the final neuronal identity acquired by that neuron, like the time of birth and/or the laminar position in the cortex etc.

\subsubsection{The effect on Unc5h3 expression}

We have shown that one way by which Ctip2 is acting involves the repression of Unc5h3. This is indicated by three main lines of evidence. First, the mRNA expressions of Ctip2 and Unc5h3 are mutually exclusive in the forebrain of neonatal mice. Second, the lack of Unc5h3 expression in the cortex observed in the Satb2 deficient brains is reversed upon the additional deletion of Ctip2 (Ctip2 $2^{-/-}$;Satb2 $2^{-/}$). Third, Ctip2 protein was shown to directly bind to Unc5h3 promoter elements and repress its expression in vivo and in vitro. These evidence, combined with the fact that Unc5h3 is largely expressed in Satb2 positive cells, show that the robust downregulation of Unc5h3 observed in the Satb2 deficient neocortex was not a direct result of the lost of Satb2 but rather an indirect consequence of the ectopic upregulation of Ctip2 in the Satb2 mutants. In other words, Satb2 is conferring callosal neuron identity, at least in part, by repressing Ctip2 expression thereby 
alleviating the repression that Ctip2 exerts on Unc5h3. The latter repression seems to take place in the basal ganglia as well, where both mRNAs are also expressed in a mutually exclusive way (Fig 15 and 16). The basal ganglia is the tissue where the in vivo binding of Ctip2 on Unc5h3 promoter was shown (Fig. 23), probably due to the fact that Ctip2 expression in the basal ganglia is much more robust (MSN Ctip2+ cells comprise $\sim 95 \%$ of striatal neurons) compared to the number of Ctip2+ cells found in the neocortex. Interestingly, Unc5h3 expression can be found in other parts of the embryo where Satb2 is also highly expressed, like the branchial arch at E9.5 and the structures that are later derived from it, e.g. jaw and cartilages of the palate (Przyborski et al., 1998). This indicates that Satb2 might also work through Unc5h3 to define proper cell identities in additional parts of the embryo, except the cortex.

\subsubsection{The effect on DCC expression}

Despite the restoration of Unc5h3 expression observed in the Ctip $2^{-/-} ; \mathrm{Satb2}^{-/}$, most of the callosal neurons are still sending abnormal projections to subcortical targets. As expected, there are additional factors that contribute to this misprojection. We have shown that DCC mRNA is upregulated in the upper cortical layers of the $\mathrm{Satb}^{-/}$by P0 a time point when normal DCC expression, especially in the caudal cortex, is almost completely absent (weak and diffused signal throughout the CP, Fig. 22). The above DCC upregulation persists in the Ctip2 $2^{-/-} ; S_{a t b 2^{-/}}$compound mutant implying its involvement in the axonal misguidance observed in both Satb2 $2^{-/}$and $\mathrm{Ctip2}^{-/} ; \mathrm{Satb2}^{-/-}$mutants, and indicating that Satb2 is somehow repressing DCC expression in a Ctip2 independent manner. Given that DCC mediates attraction to netrin-1 signals, one hypothesis would be that the expression of DCC is not needed as development of the cortex progresses because the later born neurons, that are destined to form callosal projections, do not require attraction to the internal capsule where netrin-1 is expressed.

Nonetheless, DCC protein has been shown to be expressed initially (E14.5) in axons that project laterally, while later on (E16.5) is detected in medially projecting axons (Shu, T. et al., 2000), indicating that the role of DCC is rather more complicated and probably involves a tight spatiotemporal regulation of both mRNA and protein expression. The fact that DCC protein is detected in the axons of medially projecting neurons might be due to the fact that netrin-1, except for the basal ganglia, 
is also expressed in the midline. Therefore, DCC is probably necessary for the attraction of callosal axons toward the midline; hence even though the mRNA expression is downregulated around E16.5, DCC protein is present at this stage in the axons of, at least some, medially projecting neurons. The expression of DCC protein, however, is very weak by E18 and confined only to the most dorsal axons of the corpus callosum (Shu, T. et al., 2000). Given that DCC is transported from intracellular pools to the plasma membrane (Bouchard, J-F. et al., 2004; Bouchard, JF. et al., 2008), where it binds to translation machinery (Tcherkezlan, J. et al, 2010), it is highly likely that the temporal regulation of the translation and/or transport of the protein play an important role. The relocation and stabilization of DCC protein to the growth cone changes the sensitivity of axons to netrin-1, thereby regulating their responses accordingly. Furthermore, presenting the DCC receptor to the plasma membrane is probably required not only for the transduction of the attractive signal, but also for the formation of a receptor complex with Unc $5 \mathrm{~h} 3$ that might be necessary for switching attraction to repulsion. Conversely, activation of certain kinases leads to the internalization of Unc5 homologues (Bartoe et al., 2006) thereby changing repulsion to attraction. This highly dynamic interplay is, at least partially, regulated through receptor trafficking that might mediate a faster and more efficient response to changes in environmental cues.

Fig. 34. Model of the molecular players that contribute to the specification of neuron projection identity in the forebrain.

In wt brains (A) the presence of Unc5h3 in the cortex combined with the absence (or the low, dispersed expression) of DCC leads to callosal neurons projecting to the midline and forming the C.C.. In Satb2 mutants (B) Unc5h3 expression is lost and at the same time DCC is upregulated in the UL of the cortex leading the Satb2- axons to project subcortically instead of calosally. Unc5h3 mediated repulsion to netrin-1 is lost, while DCC mediated attraction to netrin-1 (found in the basal ganglia) is induced. In Ctip2 mutant brains the presence of Unc5h3 and the absence of DCC lead to the projection of callosal neurons toward the midline (C) similar to wt brains. Lastly, the Satb2 ${ }^{-/} ; \mathrm{Ctip}^{-/}$compound mutants (D) that regain Unc5h3 expression, still have most axons projecting subcotrically (high DCC expressing axons, black) because of the persisting upregulation of DCC in the UL. However, a number of axons in the compound mutant do project toward the midline (DL, Unc5h3 expressing axons, green).

The presence of Satb2 in callosal neurons results in the repression of Ctip2, thereby alleviating the repressive effect that Ctip2 exerts on Unc5h3. At the same time Satb2 is repressing DCC expression in callosal neurons. On the other hand, Ctip2 is inhibiting Unc5h3 in subcortical projection neurons. In 
the absence of Satb2, Ctip2 is ectopically upregulated in callosal neurons where Unc5h3 is in turn repressed leading to axonal missprojection. At the same time the repression that Satb2 exerts on DCC is lost, resulting in the upregulation of DCC in superficial layers.

A Satb2 ${ }^{+/+} ; \mathrm{Ctip2}^{+/+}$

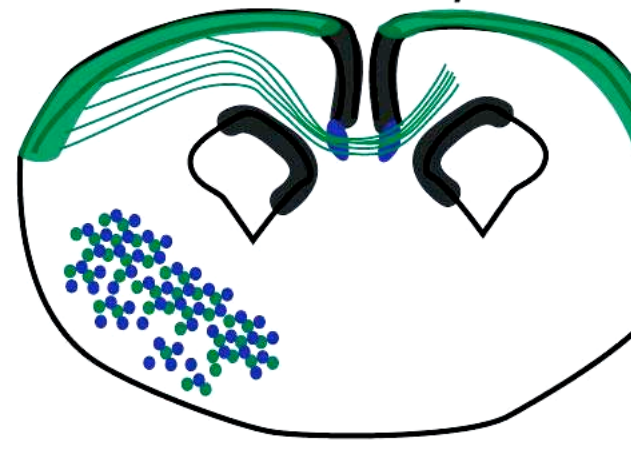

C Satb2 $2^{+/+} ;$Ctip2 $^{--}$Dcc

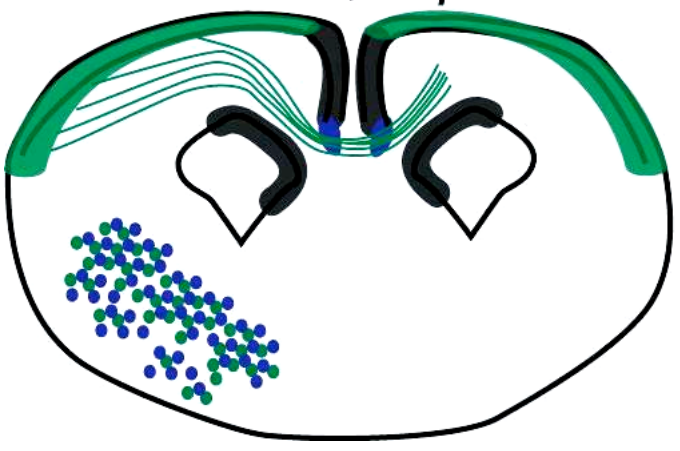

Satb2

Ntr1

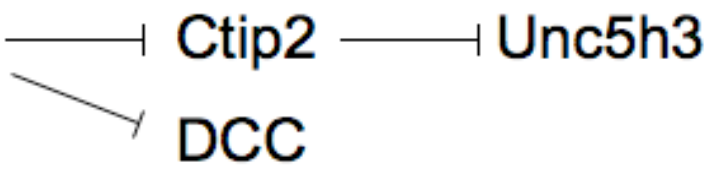

$\mathrm{Satb2}^{-1-} ; \mathrm{Ctip2}^{+/+}$B

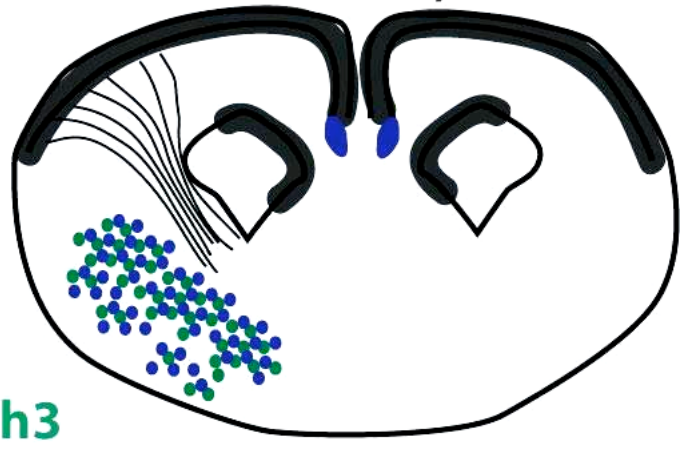

Unc5h3

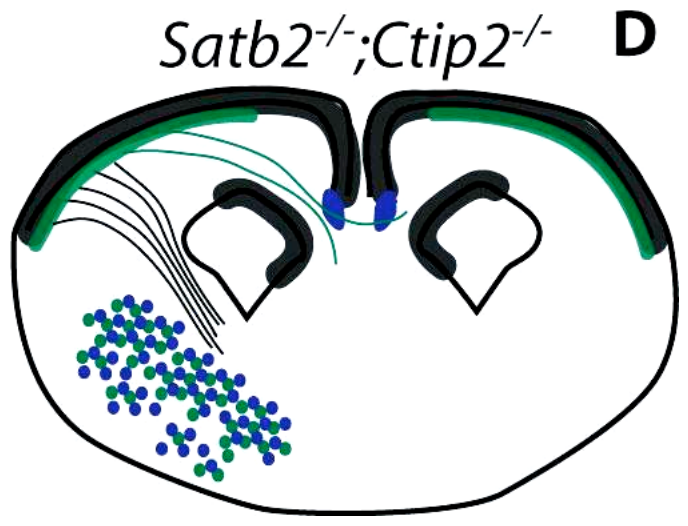

Satb2 $2^{--} ; \mathrm{Ctip2}^{-1}$ D

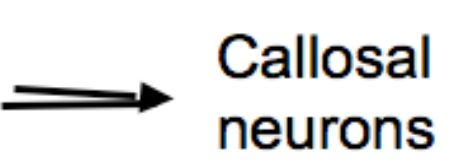




\subsubsection{Hints for the differential regulation of CPN identity amongst the different cortical layers}

The fact that in the Satb2 deficient cortex there are certain molecules whose expression is robustly downregulated throughout the whole CP, implies for a possible crucial role of these factors in the proper axon guidance of callosal projecting neurons. In order to address this possibility we decided to reintroduce in the Satb2 deficient cortex these molecules, the expression of which was shown to be almost completely lost, and examine the effects that they might have on the axonal projections of the Satb2 $2^{-/}$. Even though most callosal neurons reside in the upper cortical layers that are born at E14.5 onwards, we did not observe any obvious alterations in the axonal projections after introduction of Unc5h3 at the above developmental stage. This result implies that during the generation and/or specification of upper layer neurons, there are additional changes (except for the lack of Unc5h3) that have occurred which can override the reintroduction of Unc5h3 into the superficial Satb2 deficient cortex.

Although the majority of callosal neurons reside in upper cortical layers there is still a small percentage, which can be found in the deep layers (mainly layer $\mathrm{V})$ that are born earlier in development (around E12.5). The introduction of Unc5h3 into the mutant neocortex at E12.5 revealed a number of axons projecting toward the midline something that was not observed in control experiments. These results point to the fact that the neurons responsible for giving the above callosal projections are deep layer neurons whose fate was, at least partially, altered either at the progenitor or at, most likely, the postmitotic level. Alternatively, these cells might be deep layer neurons that normally form subcortical projections, although this possibility seems less likely. One would expect that it is much easier, and therefore more possible, for a neuron that was normally destined to become a callosal neuron to regain aspects of its lost proper identity than for a neuron destined to form subcortical projections to completely change its fate.

Interestingly, the above role in defining callosal identity cannot be attributed to all molecules the regulation of which has been shown to be controlled by Satb2 and their expression to be greatly reduced in the Satb2 deficient cortex. On the contrary, it seems to be rather specific to Unc5h3 (at least amongst the molecules tested in this study) a feature, which combined with the fact that Unc5h3 is an axon guidance 
molecule and not a transcription factor, and that the fate transformation is only partial makes it even less likely to act on the progenitor level. Even more importantly, the stage specificity of the above partial rescue points to the fact that there are different molecular mechanisms that govern the acquisition of proper neuronal identity amongst neurons that form similar projections but reside in different laminar positions. The upregulation of DCC only in the superficial layers of the Satb2 $2^{-/}$and $\mathrm{Ctip}^{-/} ; \mathrm{Satb2}^{-/-}$mutants is one additional indication for the existence of a differential regulation of gene expression between deep and upper layer callosal projecting neurons. Even though Satb2 expression is lost from all the callosal neurons, only some upregulate DCC and therefore solely those born between E12.5 and E14.5 can partially regain their callosal identity. In recent years many factors that are preferentially expressed in specific subtypes of callosal projection neurons have been identified (reviewed in Fame et al., 2011). These indications will help in the delineation of subpopulation specific genetic programs amongst neurons with similar projections but different birthdates and laminar positions.

\subsubsection{The necessity for a dynamic regulation of Netrin-1 receptors' expression throughout development}

The study of the expression patterns of two main netrin-1 receptors, Unc5h3 and DCC, has revealed a dynamic and spatiotemporal regulation of their expression throughout development.

\subsubsection{The dynamic expression of DCC and its possible role in axonal guidance}

At E14.5, a stage when most deep layer neurons are already born and have started projecting their axons to subcortical targets, the expression of DCC mRNA is robust in the developing cortical plate, mostly in the upper part (Fig. 23). Although not tested at earlier stages DCC mRNA expression is predicted to be present at least as early as E12.5 (Gad, et al. 1997), since DCC protein can already be detected by E13 in the intermediate zone of the neocortex and in the cingulate cortex (Shu et al., 2000). As development progresses DCC is downregulated in the neocortex, at least in part by Satb2, so that by P0 DCC mRNA shows only a limited expression in the 
superficial part of the rostral cortex (data not shown; Gad, et al., 1997) and is almost completely gone from the caudal cortex (Fig. 22). The observation that DCC expression in the hippocampus, where Satb2 is not normally expressed at this stage (P0), remains unaffected while in the upper cortical layers it is upregulated in a pattern that resembles Satb2 expression is reinforcing the notion that Satb2 represses DCC. The above downregulation of DCC mRNA expression in wt mice takes place between E14.5 and E16.5 and is in accordance with previously published expression patterns (Gad et al., 1997). At E14.5 and E15.5, DCC protein is highly expressed on axons within the internal capsule and starts to be downregulated by E16.5 in the same structure, while it remains in medial aspects of the neocortex and the cingulate cortex (Shu et al., 2000). By E17.5, DCC protein can only be detected in the medially projecting axons and within the corpus callosum where it also starts to gradually decline, so that by E18 it is present only in the most dorsal axons of the corpus callosum.

This dynamic spatiotemporal regulation of DCC is probably suggestive of its role in mediating axonal attraction. Given that there are two main sources of netrin-1 in the forebrain, the internal capsule and the midline, DCC seems to be regulated in such a way that it can mediate attraction of lateral projection neurons to netrin-1 in the internal capsule from E12.5 to around E15.5 while later on, E16.5 onwards, DCC mediates attraction of callosal axons to the netrin-1 source in the midline. As axons progressively reach their target they start to downregulate DCC and loose responsiveness to their attractant (netrin-1) similar to what has previously been shown for embryonic cortical neurons that undergo a netrin dependent downregulation of DCC via the ubiquitin-proteosome pathway (Kim et al, 2005). It is possible that the DCC mRNA is translated in certain neurons during specific developmental stages, or alternatively it is retained in intracellular pools and transported to the axons only when needed. In that way, the projections of different axons (subcortical and callosal) can be regulated accordingly in response to the activation by environmental cues that are localized within different regions of the forebrain.

\subsubsection{The possible role of Unc5h3 in callosal projections during development and in relation to $\mathrm{DCC}$}

Unc5h3 mRNA, on the other hand, is still not detectable at E14.5 in the cortex 
although evident in other parts of the forebrain (Fig. 23). At this stage the upper layer callosal neurons have just started to be born and only later will they extent axons toward the midline. Therefore, the presence of Unc5h3 will be necessary at a later stage to mediate repulsion to netrn-1 expressed in the internal capsule. It is possible that the presence of DCC is required in those callosal neurons that cross the midline first, in a process mediated by DCC/netrin-1 attraction to the midline while the later callosal axons, after having been repelled by Unc5h3/netrin-1 repulsion to the internal capsule, are able to follow the trajectories of the already existing callosal projections. It is known that there is a regional specificity underlying the sequential projection of callosal axons with the lateral neurons sending projections first, that cross the ventral part of the C.C., and the medial axons following them in the dorsal part of the corpus callosum. This also becomes evident from our double injection experiments in which green axons (earlier born, more lateral) are crossing the ventral C.C. while red (later born, more medial) are seen in the dorsal C.C. (Fig. 19M).

Furthermore, the high lateral to low medial expression of Unc5h3 in the cortex (our data) could serve the purpose of Unc5h3, alone or in excess compared to DCC, mediating a short-range repulsion while the combination of Unc5h3 and DCC is mediating a long-range repulsion. Therefore, in the first case the abundance of Unc5h3 in lateral cortex can efficiently repel the callosal axons of these neurons from the internal capsule, which is found in close proximity to them, while in more dorsomedial neurons the presence of both receptors becomes necessary in order for the long-range repulsion to be mediated.

It has been shown, in various cases, that the ratio of Unc5 to DCC dictates the type of responses that neurons will have by regulating the directionality of the growth cone (Muramatsu et al., 2010; Manitt et al., 2010). Therefore, it is likely that in order for Unc5h3 to mediate the repulsion necessary for dorsal neurons to project callosaly, the presence of DCC is also required since the expression of both receptors is known to mediate repulsive responses. However, due to the robust upregulation of DCC in the superficial neocortex the restoration of Unc5h3 might not be enough to override a certain Unc5h3/DCC ratio that might be required in order to switch the attractive response to repulsive. All of the above, present interesting hypotheses that remain to be tested. Nonetheless, what becomes clear from these results is that the regulation of at least two netrin-1 receptors (Unc5h3 and DCC) takes place in a precise and tightly controlled spatiotemporal manner, in such a way that the appropriate responses of the 
projecting axons to the environmental cues presented to them, at different stages, are ensured.

\subsubsection{The possible involvement of additional receptors}

The observation that even after the deletion of the ectopically expressed Ctip2, from the UL of the $S a t b 2^{-/}$cortex, there is only a partial restoration of callosal projections is indicative of the fact that Ctip2 is not the only and/or main factor that gives to the UL, Satb2 deficient, neurons their ectopic subcortical identity. There is of course, as described above, a parallel upregulation of DCC expression in the superficial cortex of the Satb2 $2^{-/} ; \mathrm{Ctip}^{-/-}$compound mutants which can, at least partially, explain the lack of callosal projecting UL neurons despite the Unc5h3 restoration. Except for the above netrin-1 receptors, however, there are additional factors that have been shown to play roles in netrin-1 signaling the misexpression of which might be also responsible for the observed projection defects. Neogenin has been known for a while to be another member of the DCC family, but only recently has its function started to be revealed. The largely, but not completely, nonoverlapping expression patterns with DCC imply for a possible lack of functional redundancy. While acting as an attractant for netrin-1, neogenin is also acting as a repellent for repulsive guidance molecules (RGMs), a ligand that does not belong to the netrin-1 family. Addiotionally, the intriguing emerging roles of DSCAM and heparin in mediating netrin-1 signaling (see introduction), are making the examination of their possible involvement in axonal guidance, in vivo, a worthwhile study.

\subsection{Regulation of subcortical projections}

The reports, by Chen et al. 2008 and the more recent one by Lodato et al. 2011, that there is an ectopic upregulation of Satb2 expression in the deeper layers of the $\mathrm{Fezl}^{-/}$led to the conclusion that the deep layer cells acquire an abnormal callosal identity which results in the lack of subcerebral projection neurons. This notion was reinforced by the observation that Fezl mutant neurons project accross the C.C. in chimeric mice (Chen et al., 2008) and in Fezl mutants, shown by retrograde labeling of CPNs from the contralateral cortex (Lodato et al., 2011). 


\subsubsection{Possible existence of different molecular determinants within distinct subpopulations of DL neurons}

In order to address the question of whether the absence of the ectopically expressed Satb2 in the deep layers of the $\mathrm{Fezl}^{-/}$can make these neurons return to their original subcortical identity, we decided to generate $\mathrm{Fezl}^{-/} ; \mathrm{Satb}^{-/-}$compound mutants. Examination of these animals showed that there was only a partial rescue in the subcortical projecting neurons with an increase in the number of axons transversing the C.P. but no restoration of the CST. This result clearly suggests that the presence of Fezl, and thereby Ctip2, at least for CST deep layer neurons is indispensable for the proper specification of their projections.

The fact that there is a restoration of Ctip2 expression in the compound mutants, at least in the low Ctip2 expressing DL cells, is indicative of a possible role for Satb2 in mediating the identity of the non-CSMN neurons of layer V. In these neurons, Fezl seems to mediate their Ctip2 expression through inhibiting Satb2, which when lost, the repression that Satb2 exerts on Ctip2 is alleviated as well, thereby allowing for Ctip2 expression to be regained. Since it seems rather unlikely that Ctip2 expression is restored, at least not fully, in the type I layer V neurons, it becomes clear that these neurons need Fezl for their proper specification, which, at least for the most part, is taking place via Ctip2 in a Satb2 independent manner. Nonetheless, even if the Ctip2 expression in these cells (CSMN) is partially restored, it is certain that they cannot be fully transfated because major aspects of their identity, like their projection to the CST, are still missing. Although not directly addressed, the Ctip2 positive cells in the compound mutant seem to suffer migration defects (a feature which is anyway present in the Satb2 mutants), given that the neurons are distributed in a rather disorganized manner within the deep cortical layers. This might be an additional reason why the presence of Ctip2 expression in the deep layers of the compound mutants is not enough to give these neurons their full subcerebral identity.

Given that there is a number of ectopically expressing Ctip2 cells that project subcortically in the UL of the Satb2 mutants, the partial restoration observed in the $\mathrm{Satb2}^{-/} ; \mathrm{Fezl}^{-/}$compound mutants could be the result of those projections, since the Ctip2 ectopic expression in UL persists in the double mutant brains. As shown by our backlabeling experiments, there are indeed cells of upper layers that project

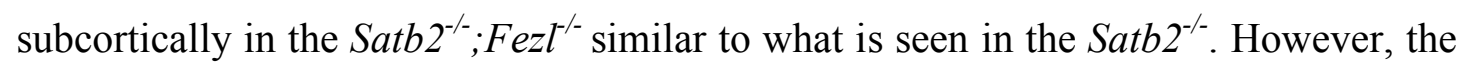


same experiments revealed that in the $\mathrm{Satb2}^{-/} ; \mathrm{Fezl}^{-/}$there are also many backlabeled cells that are found in deep cortical layers, clearly pointing to the fact that at least some of the subcortical projecting DL neurons regain aspects of their subcerebral connectivity.

On the other hand, the deep layer neurons that manage to project subcortically in the compound mutants have those altered phonotypic characteristics previously described for the deep layer neurons of the Fezl--mice (Chen et al., 2008). Specifically, these neurons depict an altered and rather disorganized dendritic morphology and their apical dendrites show significantly fewer, if any, terminal branches. The above results in combination with alterations in the expression of markers for subcortical projecting neurons (e.g. FNP-7) on one hand, and the, at least partial, restoration of the expression of subcerebral projection markers on the other hand, make it clear that there are different regulatory mechanisms that govern the specification of different subtypes of subcortical projection neurons. The presence of certain molecules, like Fezl, might be indispensable for some neuronal types (e.g. CSMN), while for others it might be less important (e.g. non-CSMN subcortical neurons). The above differences are most likely also accompanied by differences in the way those molecules exert their effects on distinct neuronal subtypes, eg. via a pathway either dependent or independent of Satb2. In any case one should keep in mind that there is certain evidence which suggests that Fezl is very likely to also act through other mechanisms that are entirely independent not only of Satb2 but of Ctip2 as well. This becomes more evident by the differences in severity between the Fezl and the Ctip2 mutant phenotypes, with the former missing almost completely all subcerebral projections while the latter is extenting axons all the way to the pons but never to the spinal cord.

\subsubsection{Ectopic subcortical identity of upper layers is regulated independently of} Fezl

Except for the lack of a full restoration of Ctip2 expression, and therefore of subcortical connectivity, in the deep layers of the $\mathrm{Satb2}^{-/} ; \mathrm{Fezl}^{-/}$compound mutants there is also a persisting ectopic expression of Ctip2 in the upper cortical layers. These upper layer cells seem to maintain most aspects of their, ectopic subcortical identity, since, as shown by our experiments, they continue to sent projections to 
subcerebral targets. The above result points to the fact that Ctip2 expression in the UL of the compound mutants is regulated in a way, which is independent of Fezl. In other words, it is the absence or the presence of the post-mitotic determinant Satb2 that dictates the expression or not, respectively, of Ctip2 in upper cortical layers. The influence of Fezl, even though it acts at the progenitor level, is not evident in the mechanisms concerning the callosal neuron identity. 


\section{CONCLUSION}

The complex molecular mechanisms that govern axonal guidance and neuronal connectivity share many common features and at the same time diverge characteristics amongst different organisms. Even more interesting is the differential regulation of the cellular identities and therefore properties observed within the same tissue or even amongst neurons of similar topology that share many common features. In the current study we sought to reveal some of the mechanisms that guide callosal neurons to their appropriate targets and are responsible for the important intercerebral connectivity. We have elucidated, in more detail, the way by which Satb2 delineates callosal neuron identity and have identified aspects of its downstream pathway. More specifically, we have shown that Satb2 regulates Unc5h3 expression by repressing Ctip2 in callosal neurons, thereby alleviating the repression of Ctip2 on Unc5h3. Moreover, we have shown that Satb2 also exerts a repressive effect on DCC through a yet unknown mechanism.

The important role of Unc5h3 in guiding callosal axons was demonstrated by overexpression experiments that resulted in a partial restoration of callosal projections in the $\mathrm{Satb2}^{-/}$, as well as by the observation of a similar partial rescue in the Ctip $2^{-/-}$ ;Satb2 ${ }^{-/}$compound mutants. Furthermore, the stage specificity of the above rescue and the lack of a more complete restoration of callosal projections in the Ctip2 ${ }^{-/}$ ;Satb2 ${ }^{-/}$argue for the differential regulation of gene expression amongst neurons that form similar projections but do not share the same topology (UL and DL callosal CPNs). This study revealed an, up to now, unknown convergence of the Satb2 and the netrn-1 pathways.

The divergence amongst CPNs also applies to subcortical projection neurons which even though they are almost completely lost in the $\mathrm{Fezl}^{-/}$, a subset of them is shown to partially regain aspects of their connectivity in the $\mathrm{Fezl}^{--} ; \mathrm{Satb}^{-/}$. All of the above reinforce the existing notion of the diverse molecular profile amongst neurons with similar projections but different laminar positions and extend some further evidence to a more mechanistic insight.

The delineation of the molecular mechanisms that govern proper axon guidance, and thereby correct connectivity, is an interesting an ambitious task that would most likely keep producing more questions than answers. 


\section{APPENDIX}

\subsection{Expression of axon guidance molecules and transcription factors in the Satb2-}

\section{${ }^{\prime}$ Ctip $2^{-/}$}
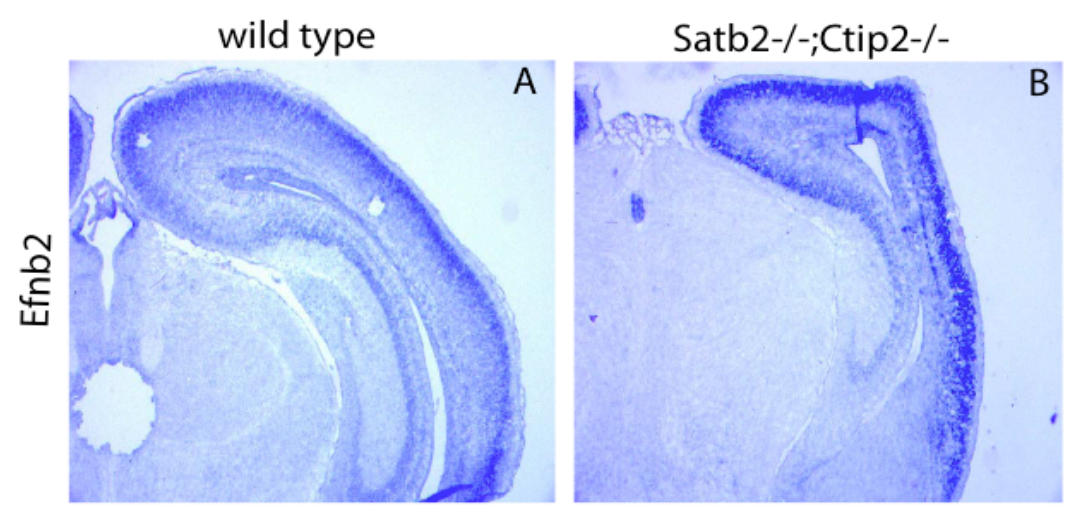

In the Satb2 $2^{-1}$

;Ctip2 ${ }^{-/-}$compound

mutants there are

additional axon

guidance molecules

or transcription

factors the
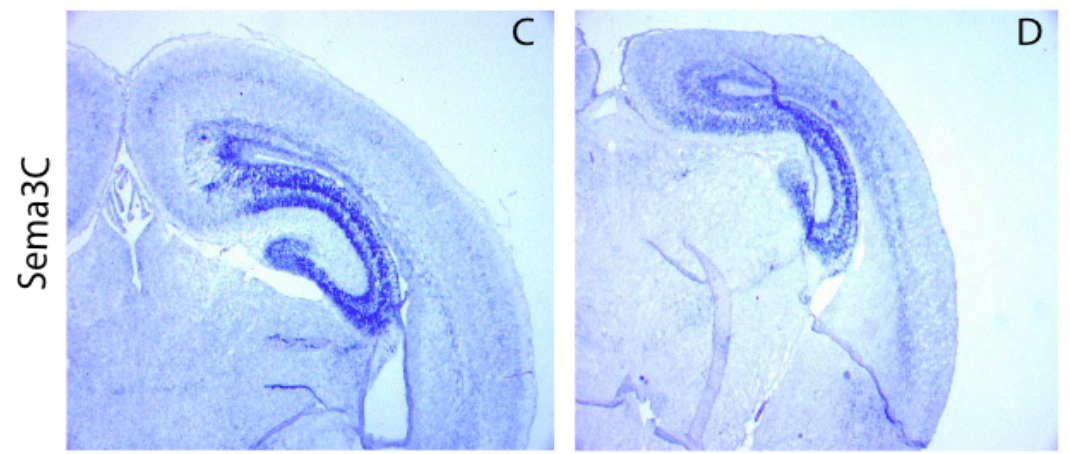

expression

of

which is altered

(e.g.

Efnb2,

EphB1) and others

that remain

unchanged (e.g.
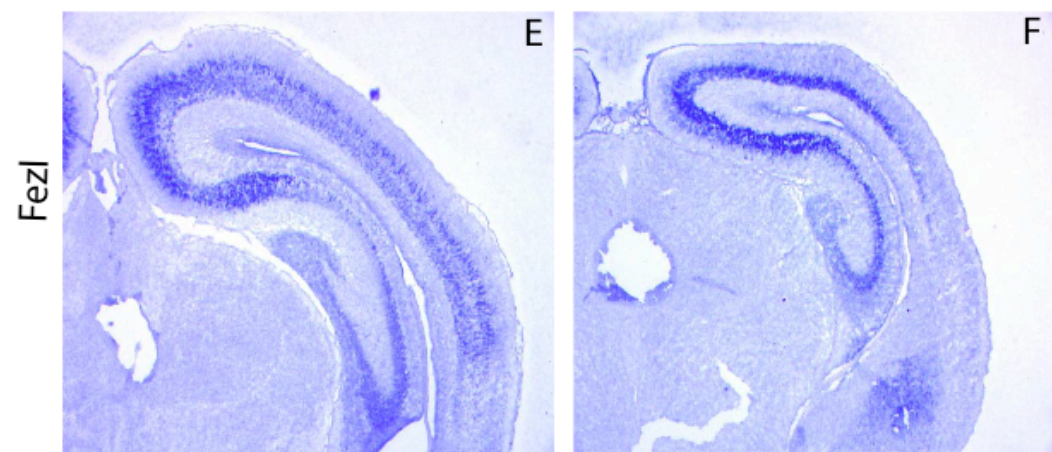

F
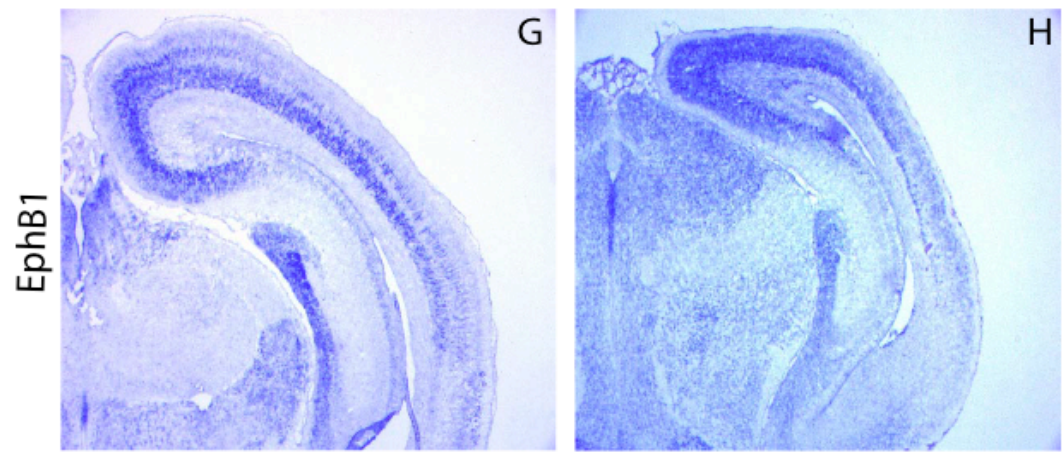

Fezl, Sema3C).

The significance of such alterations in regulating callosal projections remains to be elucidated. 
Fig. 35. Expression patterns in the $\mathrm{Ctip2}^{-/} ; \mathrm{Satb}^{-/}$compound mutants. Chromogenic in situ hybridization of axon guidance molecules and transcription factors in wt and compound mutants. All brains were isolate at P0. Ephrinb2 (B) and EphB1 (H) are upregulated in the superficial layers of the double mutant compared to wt cortices (A and G). Sema3C and Fezl show unaltered expression patterns in the compound mutants (D and F) compared to wt brains (C and $\mathrm{E})$.

Except for the netrin-1 receptors Unc5h3 and DCC additional axon guidance molecules, the expression of which is shown to be altered in the P0 Satb2 deficient cortex, do not show any changes earlier in development. For example the axon guidance molecule PlxA4, which is greatly downregulated in the cortical plate of the $\mathrm{Satb}^{-/}$has normal expression in all three different mutants examined at E14.5. It seems that, in general, the effects of Satb2 and/or Ctip2 deletion on the expression patterns of various molecules do not become apparent until later in development.
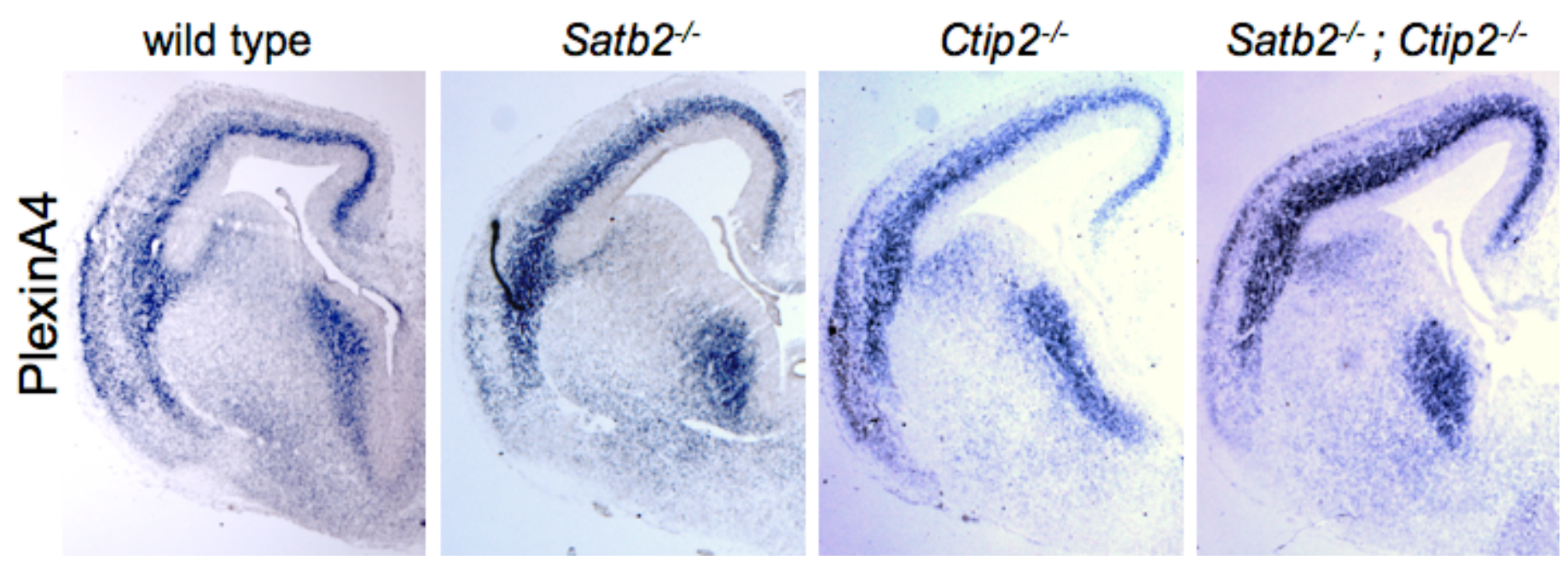

Fig. 36 Expression pattern of PlexinA4 is not influenced by Satb2 and/or Ctip2 deletion at early

stages. Chromogenic in situ hybridization for PlexinA4 on coronal sections of E.14.5 brains in the four different genotypes depicted. 


\subsection{Ephrinb2 overexpression in wt cortex}

Except for the molecules that show a robust dowregulation in the whole cortical plate of the $\mathrm{Satb2}^{-/}$there are also some axon guidance molecules that have been shown to be upregulated in specific parts of the CP (Table 1). One example of these molecules is ephrinb2, which is distinctly upregulated in the superficial cortical plate of the Satb2 $2^{-/}$. In order to examine whether these molecules can phenocopy some aspects of the misprojections observed in the $S a t b 2^{-/-}$overexpression experiments, via in utero electroporation, were performed in wt animals at different embryonic stages. Below, the overexpression of ephrinb2 at E12.5 can be seen in wt brains that were harvested at P0. Although no apparent misprojection of axons could be detected, a closer look at the ephrinb2 overexpressing cortical cells reveals a clustering and an abnormal laminar distribution (Fig. 37B'). Staining with Ctip2 shows that the Ctip2 positive cells are not positioned properly in layer V but are found within these clusters in which many cells are depicting delayed migration. This result shows a possible role for ephrinb2 in cell adhesion and/or migration of cortical neurons. Injection at a later stage (E14.5) did not reveal the same effect (data not shown).

Fig. 37 Overexpression of ephrinb2. In utero electroporation, with venus-GFP (A-D) and ephrinb2GFP (A'-D"), at E12.5 in wt brains that were harvested at P0. Although no detectable axonal misguidance could be found after ephrinb2 injection (A') the cortical cells overexpressing ephrinb2 formed clusters and had abnormal migration (B'), implying a possible role for ephrinb2 in cell adhesion and/or migration. The ctip $2+$ cells for example were seen in various layers resembling cells that experienced impaired migration due to clustering (D'). 


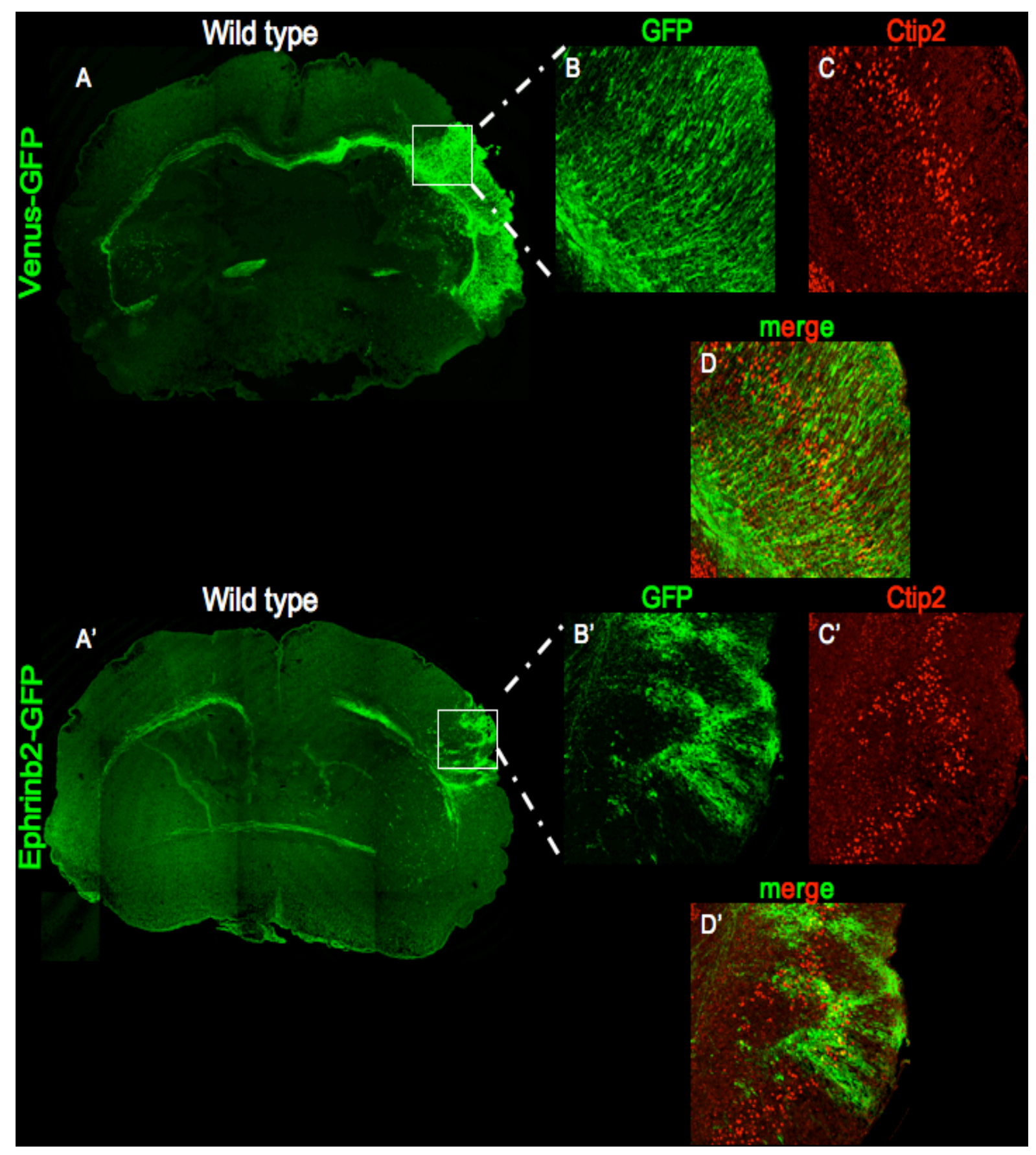




\section{REFERANCES}

Ackerman, S. et al. (1997). The mouse rostral cerebellar malformation gene encodes an Unc-5-like protein. Nature 386, 838-842

Alcamo, E.A., Chirivella, L., Dautzenberg, M., Dobreva, G., Farinas, I., Grosschedl, R., and McConnell, S.K. (2008). Satb2 regulates callosal projection neuron identity in the developing cerebral cortex. Neuron 57, 364-377

Alcántara S, Ruiz M, De Castro F, Soriano E, Sotelo C. (2000) Netrin 1 acts as an attractive or as a repulsive cue for distinct migrating neurons during the development of the cerebellar system. Development. 127(7), 1359-72.

Andrews GL, Tanglao S, Farmer WT, Morin S, Brotman S, Berberoglu MA, Price H, Fernandez GC, Mastick GS, Charron F, Kidd T. (2008) Dscam guides embryonic axons by Netrin-dependent and -independent functions. Development. 135(23), 383948

Angevie., J. B. Jr and Sidman, R. L. (1961). Autoradiographic study of cell migration during histogenesis of cerebral cortex in mouse. Nature 192, 766-768

Arlotta et al. (2005). Neuronal subtype-specific genes that control corticospinal motor neuron development in vivo. Neuron 45(2), 207-221

Arlotta P, Molyneaux BJ, Jabaudon D, Yoshida Y, Macklis JD. (2008). Ctip2 controls the differentiation of medium spiny neurons and the establishment of the cellular architecture of the striatum. J Neurosci. 28(3), 622-32

Avram, D., Fields, A., Pretty On Top, K., Nevrivy, D. J., Ishmael, J.E. and Leid, M. (2000). Isolation of a novel family of $\mathrm{C} 2 \mathrm{H} 2$ Zinc finger proteins implicated in transcriptional repression mediated by Chichen Ovalbumin Upstream Promoter Transcription Factor (COUP-TF) orphan nuclear receptors. The Journal of Biological Chemistry 275(14), 10315-10322

Avram D, Fields A, Senawong T, Topark-Ngarm A, Leid M. (2002). COUP-TF (chicken ovalbumin upstream promoter transcription factor)-interacting protein 1 (CTIP1) is a sequence-specific DNA binding protein. Biochem J. 368, 555-63.

Azim E, Shnider SJ, Cederquist GY, Sohur US, Macklis JD. (2009). Lmo4 and Clim1 progressively delineate cortical projection neuron subtypes during development. Cereb Cortex. 62-9.

Bartoe JL, McKenna WL, Quan TK, Stafford BK, Moore JA, Xia J, Takamiya K, Huganir RL, Hinck L. (2006). Protein interacting with C-kinase 1/protein kinase Calpha-mediated endocytosis converts netrin-1-mediated repulsion to attraction. J Neurosci. 26(12), 3192-205

Berberoglu MA, Dong Z, Mueller T, Guo S. (2009). fezf2 expression delineates cells with proliferative potential and expressing markers of neural stem cells in the adult 
zebrafish brain. Gene Expr Patterns 9(6), 411-22

Bielle F., Griveau A., Narboux-Neme N., Vigneau S., Sigrist M., Arber S., Wassef M., and Pierani A. (2005). Multiple origins of Cajal-Retzius cells at the borders of the developing pallium. Nat Neurosci 8, 1002-1012.

Blechman J, Borodovsky N, Eisenberg M, Nabel-Rosen H, Grimm J, Levkowitz G. (2007). Specification of hypothalamic neurons by dual regulation of the homeodomain protein Orthopedia Development. 134(24), 4417-26.

Bloch-Gallego E, Ezan F, Tessier-Lavigne M, Sotelo C. (1999) Floor plate and netrin1 are involved in the migration and survival of inferior olivary neurons. J Neurosci. 19(11), 4407-20.

Bouchard JF, Moore SW, Tritsch NX, Roux PP, Shekarabi M, Barker PA, Kennedy TE. (2004). Protein kinase A activation promotes plasma membrane insertion of DCC from an intracellular pool: A novel mechanism regulating commissural axon extension. J Neurosci. 24(12), 3040-50.

Bouchard JF, Horn KE, Stroh T, Kennedy TE. (2008). Depolarization recruits DCC to the plasma membrane of embryonic cortical neurons and enhances axon extension in response to netrin-1. J Neurochem. 107(2), 398-417.

Bradford D. Faull RL, Curtis MA, Cooper HM. (2010). Characterization of the Netrin/RGMa receptor neogenin in neurogenic regions of the mouse and human adult forebrain. J Comp Neurol. 518(16), 3237-53.

Braisted JE, Catalano SM, Stimac R, Kennedy TE, Tessier-Lavigne M, Shatz CJ, O'Leary DD.(2000). Netrin-1 promotes thalamic axon growth and is required for proper development of the thalamocortical projection. J Neurosci. 20(15), 5792-801.

Britanova, O. et al. (2008). Satb2 is a postmitotic determinant for upper-layer neuron specification in the neocortex. Neuron 57(3), 378-392

Britanova, O. et al. (2006). Satb2 haploinsufficiency phenocopies 2q32-q33 deletions, whereas loss suggests a fundamental role in the coordination of jaw development. Am J Hum Genet 79(4), 668-678

Britanova O, Alifragis P, Junek S, Jones K, Gruss P, Tarabykin V. (2006). A novel mode of tangential migration of cortical projection neurons. Dev Biol. 298(1), 299311.

Britanova, O., Akopov, S., Lukyanov, S., Gruss, P. and Tarabykin, V. (2005). Novel transcription factor Satb2 interacts with matrix attachment region DNA elements in a tissue-specific manner and demonstrates cell-type-dependent expression in the developing mouse CNS. Eur. J. Neurosci. (21)3, 658-68

Bulchand S, Subramanian L, Tole S (2003). Dynamic spatiotemporal expression of LIM genes and cofactors in the embryonic and postnatal cerebral cortex. Dev Dyn. 226(3), 460-9. 
Burgess RW, Jucius TJ, Ackerman SL. (2006) Motor axon guidance of the mammalian trochlear and phrenic nerves: dependence on the netrin receptor Unc $5 \mathrm{c}$ and modifier loci. J Neurosci. 26(21), 5756-66.

Chen B, Schaevitz LR, McConnell SK. (2005) Fezl regulates the differentiation and axon targeting of layer 5 subcortical projection neurons in cerebral cortex.

Proc Natl Acad Sci U S A 102(47), 17184-9.

Chen B, Wang SS, Hattox AM, Rayburn H, Nelson SB and McConnell S. (2008). The Fezf2-Ctip2 genetic pathway regulates the fate choice of subcortical projection neurons in the developing cerebral cortex. PNAS 105(32), 11382-87.

Choi YS, Hong SB, Jeon HK, Kim EJ, Oh WJ, Joe SY, Han JS, Lee MJ, Lee HW. (2003) Insertional mutation in the intron 1 of Unc5h3 gene induces ataxic, lean and hyperactive phenotype in mice. Exp Anim. 52(4), 273-83.

Colamarino SA, Tessier-Lavigne M. (1995) The axonal chemoattractant netrin-1 is also a chemorepellent for trochlear motor axons. 81(4), 621-9.

Corset V, Nguyen-Ba-Charvet KT, Forcet C, Moyse E, Chédotal A, Mehlen P. (2000). Netrin-1-mediated axon outgrowth and cAMP production requires interaction with adenosine A2b receptor. Nature 407, 747-750

Desai, A. R. and McConnell, S. K. (2000). Progressive restriction in fate potential by neural progenitors during cerebral cortical development. Development 127, 28632872

De Vries M, Cooper HM. (2008) Emerging roles for neogenin and its ligands in CNS development. J Neurochem. 106(4), 1483-92

Dillon AK, Fujita SC, Matise MP, Jarjour AA, Kennedy TE, Kollmus H, Arnold HH, Weiner JA, Sanes JR, Kaprielian Z. J (2005) Molecular control of spinal accessory motor neuron/axon development in the mouse spinal cord. Neurosci. 25(44), 1011930 .

Dillon AK, Jevince AR, Hinck L, Ackerman SL, Lu X, Tessier-Lavigne M, Kaprielian Z. (2007). UNC5C is required for spinal accessory motor neuron development Mol Cell Neurosci. 35(3), 482-9.

Dobreva, G., Dambacher, J. and Grosscheld, R. (2003). SUMO modification of a novel MAR-binding protein, SATB2, modulates immunoglobulin $\mu$ gene expression. Genes and Development 17, 3048-3061

Engelkamp D. (2002) Cloning of three mouse Unc5 genes and their expression patterns at mid-gestation. Mech Dev. 118(1-2), 191-7.

Fame RM, MacDonald JL, Macklis JD. (2011). Development, specification, and diversity of callosal projection neurons. Trends Neurosci. 34(1), 41-50 
Fazeli A, Dickinson SL, Hermiston ML, Tighe RV, Steen RG, Small CG, Stoeckli ET, Keino-Masu K, Masu M, Rayburn H, Simons J, Bronson RT, Gordon JI, TessierLavigne M, Weinberg RA. (1997) Phenotype of mice lacking functional Deleted in colorectal cancer (Dcc) gene. Nature. 386(6627), 796-804.

Finger, JH. et al. (2002). The netrin 1 receptors Unc5h3 and Dcc are necessary at multiple choice points for the guidance of corticospinal tract axons. The Journal of Neuroscience. 22(23), 10346-10356

Fishell, G. and Hanashima, C. (2008). Pyramidal Neurons Grow Up and Change Their Mind. Neuron 57, 333-338

Fitzgerald DP, Cole SJ, Hammond A, Seaman C, Cooper HM. (2006)

Characterization of neogenin-expressing neural progenitor populations and migrating neuroblasts in the embryonic mouse forebrain. Neuroscience. 142(3), 703-16.

FitzPatrick DR, Carr IM, McLaren L, Leek JP, Wightman P, Williamson K, Gautier P, McGill N, Hayward C, Firth H, Markham AF, Fantes JA, Bonthron DT. (2003). Identification of SATB2 as the cleft palate gene on 2q32-q33. Hum Mol Genet. 12(19), 2491-501.

Forcet C, Stein E, Pays L, Corset V, Llambi F, Tessier-Lavigne M, Mehlen P. (2002) Netrin-1-mediated axon outgrowth requires deleted in colorectal cancer-dependent MAPK activation. Nature. 417(6887), 443-7

Frantz, G. D. \& McConnell, S. K. (1996). Restriction of late cerebral cortical progenitors to an upper-layer fate. Neuron 17, 55-61

Gad JM, Keeling SL, Shu T, Richards LJ, Cooper HM. The spatial and temporal expression patterns of netrin receptors, (2000) DCC and neogenin, in the developing mouse retina. Exp Eye Res. 70(6), 711-22.

Gal, J. S. et al. (2006). Molecular and morphological heterogeneity of neural precursors in the mouse neocortical proliferative zones. J. Neurosci. 26, 1045-1056

Geisbrecht BV, Dowd KA, Barfield RW, Longo PA, Leahy DJ. (2003) Netrin binds discrete subdomains of DCC and UNC5 and mediates interactions between DCC and heparin J Biol Chem. 278(35), 32561-8.

Goldowitz D, Hamre KM, Przyborski SA, Ackerman SL. (2000) Granule cells and cerebellar boundaries: analysis of Unc5h3 mutant chimeras. J Neurosci. 20(11), 412937

Guan Kun-Liang and Rao Yi (2003). Signaling mechanisms mediating neuronal responses to guidance cues. Nature Reviews Neuroscience. 4, 941-956

Gyorgy, A. B., Szemes, M., Romero de Juan, C., Tarabykin, V. and Agoston, D. V. (2008). Satb2 interacts with chromatin-remodeling molecules in differentiating 
cortical neurons. European Journal of Neuroscience 27, 865-873

Han W, Kwan KY, Shim S, Lam MM, Shin Y, Xu X, Zhu Y, Li M, Sestan N. (2011). TBR1 directly represses Fezf2 to control the laminar origin and development of the corticospinal tract. Proc Natl Acad Sci U S A.108(7), 3041-6

Hao JC, Adler CE, Mebane L, Gertler FB, Bargmann CI, Tessier-Lavigne M. (2010) The tripartite motif protein MADD-2 functions with the receptor UNC-40 (DCC) in Netrin-mediated axon attraction and branching. Dev Cell. 18(6), 950-60.

Hashimoto H, Yabe T, Hirata T, Shimizu T, Bae Y, Yamanaka Y, Hirano T, Hibi M. (2000). Expression of the zinc finger gene fez-like in zebrafish forebrain. Mech Dev. 97(1-2):191-5.

Hedgecock EM, Culotti JG, Hall DH. (1990). The unc-5, unc-6, and unc-40 genes guide circumferential migrations of pioneer axons and mesodermal cells on the epidermis in C. elegans. Neuron. 4(1), 61-85.

Hirata T, Suda Y, Nakao K, Narimatsu M, Hirano T, Hibi M. (2004). Zinc finger gene fez-like functions in the formation of subplate neurons and thalamocortical axons. Dev Dyn.230 (3), 546-56.

Hong, K., Hinck, L., Nishiyama, M., Poo, M., Tessier-Lavigne, M. and Stein, E. (1999). A ligand-gated association between cytoplasmic domains of Unc5 and DCC family receptors converts Netrin-induced growth cone attraction to repulsion. Cell. 97, 927-941

Jarjour AA, Manitt C, Moore SW, Thompson KM, Yuh SJ, Kennedy TE (2003). Netrin-1 is a chemorepellent for oligodendrocyte precursor cells in the embryonic spinal cord.. J Neurosci. 23(9), 3735-44.

Jeong JY, Einhorn Z, Mercurio S, Lee S, Lau B, Mione M, Wilson SW, Guo S. (2006). Neurogenin 1 is a determinant of zebrafish basal forebrain dopaminergic neurons and is regulated by the conserved zinc finger protein Tof/Fezl. Proc Natl Acad Sci U S A. 103(13):5143-8.

Jeong JY, Einhorn Z, Mathur P, Chen L, Lee S, Kawakami K, Guo S. (2007). Patterning the zebrafish diencephalon by the conserved zinc-finger protein Fezl. Development. 134(1), 127-36.

Keeling SL, Gad JM, Cooper HM. (1997) Mouse Neogenin, a DCC-like molecule, has four splice variants and is expressed widely in the adult mouse and during embryogenesis. Oncogene. 15(6), 691-700.

Keino-Masu K, Masu M, Hinck L, Leonardo ED, Chan SS, Culotti JG, TessierLavigne M. (1996) Deleted in Colorectal Cancer (DCC) encodes a netrin receptor. Cell. 87(2), 175-85.

Kenedy et al. (1994). Netrins are diffusible chemotropic factors for commissural axons in the embryonic spinal cord. Cell 78, 425-434 
Kim D, Ackerman SL. (2011) The UNC5C netrin receptor regulates dorsal guidance of mouse hindbrain axons. J Neurosci. 31(6), 2167-79.

Kim TH, Lee HK, Seo IA, Bae HR, Suh DJ, Wu J, Rao Y, Hwang KG, Park HT. (2005). Netrin induces down-regulation of its receptor, Deleted in Colorectal Cancer, through the ubiquitin-proteasome pathway in the embryonic cortical neuron. J Neurochem. 95(1), 1-8.

Komuta Y, Hibi M, Arai T, Nakamura S, Kawano H. (2007). Defects in reciprocal projections between the thalamus and cerebral cortex in the early development of Fezl-deficient mice. J Comp Neurol. 503(3), 454-65.

Kruger RP, Lee J, Li W, Guan KL. (2004) Mapping netrin receptor binding reveals domains of Unc5 regulating its tyrosine phosphorylation. J Neurosci. 24(48), 1082634.

Kubo T, Yamashita T. Rho-ROCK inhibitors for the treatment of CNS injury. (2007) Recent Pat CNS Drug Discov 2(3), 173-9

Kuninger D, Kuns-Hashimoto R, Kuzmickas R, Rotwein P. (2006) Complex biosynthesis of the muscle-enriched iron regulator RGMc. J Cell Sci. 119(Pt 16), 3273-83

Lane PW, Bronson RT, Spencer CA. (1992) Rostral cerebellar malformation, (rcm): a new recessive mutation on chromosome 3 of the mouse. J Hered. 83(4), 315-8

Leid M, Ishmael JE, Avram D, Shepherd D, Fraulob V, Dollé P. (2004). CTIP1 and CTIP2 are differentially expressed during mouse embryogenesis. Gene Expr Patterns $6,733-9$

Leonardo ED, Hinck L, Masu M, Keino-Masu K, Ackerman SL, Tessier-Lavigne M. (1997). Vertebrate homologues of C. elegans UNC-5 are candidate netrin receptors. Nature. 386(6627), 833-8

Levkowitz G, Zeller J, Sirotkin HI, French D, Schilbach S, Hashimoto H, Hibi M, Talbot WS, Rosenthal A. (2003) Zinc finger protein too few controls the development of monoaminergic neurons. Nat Neurosci. 6(1), 28-33.

Li W, Aurandt J, Jürgensen C, Rao Y, Guan KL. (2006) FAK and Src kinases are required for netrin-induced tyrosine phosphorylation of UNC5. J Cell Sci. 119(Pt 1), 47-55

Li X, Gao X, Liu G, Xiong W, Wu J, Rao Y. (2008) Netrin signal transduction and the guanine nucleotide exchange factor DOCK180 in attractive signaling. Nat Neurosci. 11(1), 28-35.

Liu G, Li W, Wang L, Kar A, Guan KL, Rao Y, Wu JY. (2009) DSCAM functions as a netrin receptor in commissural axon pathfinding. Proc Natl Acad Sci U S A. 106(8), 2951-6 
Lodato S, Rouaux C, Quast KB, Jantrachotechatchawan C, Studer M, Hensch TK and Arlotta P. (2011). Excitatory projection neuron subtypes control the distribution of local inhibitory interneurons in the cerebral cortex. Neuron 69, 763-779

Ly A, Nikolaev A, Suresh G, Zheng Y, Tessier-Lavigne M, Stein E. (2008). DSCAM is a netrin receptor that collaborates with $\mathrm{DCC}$ in mediating turning responses to netrin-1. Cell 133(7), 1241-54

Manitt C, Labelle-Dumais C, Eng C, Grant A, Mimee A, Stroh T, Flores C. (2010). Peri-pubertal emergence of UNC-5 homologue expression by dopamine neurons in rodents. PLoS One 5(7), e11463.

Marban C, Suzanne S, Dequiedt F, de Walque S, Redel L, Van Lint C, Aunis D, Rohr O (2007). Recruitment of chromatin-modifying enzymes by CTIP2 promotes HIV-1 transcriptional silencing. EMBO J. 26(2), 412-23

Masuda T, Watanabe K, Sakuma C, Ikenaka K, Ono K, Yaginuma H. (2008) Netrin-1 acts as a repulsive guidance cue for sensory axonal projections toward the spinal cord. J Neurosci. 28(41), 10380-5.

Matsumoto Y, Irie F, Inatani M, Tessier-Lavigne M, Yamaguchi Y. Netrin-1/DCC signaling in commissural axon guidance requires cell-autonomous expression of heparan sulfate. (2007) J Neurosci. 27(16), 4342-50.

Matsumoto H, Nagashima M. Netrin-1 elevates the level and induces cluster formation of its receptor DCC at the surface of cortical axon shafts in an exocytosisdependent manner. (2010) Neurosci Res. 67(2), 99-107.

Matsuo-Takasaki M, Lim JH, Beanan MJ, Sato SM, Sargent TD. (2000). Cloning and expression of a novel zinc finger gene, Fez, transcribed in the forebrain of Xenopus and mouse embryos. Mech Dev. 93(1-2), 201-4

Mawdsley DJ, Cooper HM, Hogan BM, Cody SH, Lieschke GJ, Heath JK. (2004) The Netrin receptor Neogenin is required for neural tube formation and somitogenesis in zebrafish. Dev Biol. 269(1), 302-15.

McKenna WL, Betancourt J, Larkin KA, Abrams B, Guo C, Rubenstein JL, Chen B. (2011). Tbr1 and Fezf2 regulate alternate corticofugal neuronal identities during neocortical development. J Neurosci 31(2), 549-64.

Meriane M, Tcherkezian J, Webber CA, Danek EI, Triki I, McFarlane S, BlochGallego E, Lamarche-Vane N. (2004) Phosphorylation of DCC by Fyn mediates Netrin-1 signaling in growth cone guidance. J Cell Biol. 167(4), 687-98.

Metin C, Deleglise D, Serafini T, Kennedy ET and Tessier-Lavigne M (1997). The role for netrin-1 in the guidance of cortical efferents. Development 124, 5063-5074

Niederkofler V, Salie R, Sigrist M, Arber S. Repulsive guidance molecule (RGM) gene function is required for neural tube closure but not retinal topography in the mouse visual system. (2004) J Neurosci. 24(4), 808-18. 
Molyneaux BJ, Arlotta P, Hirata T, Hibi M, and Macklis JD (2005). Fezl is required for the bith and specification of corticospinal motor neurons. Neuron 47, 817-831

Molyneaux BJ, Arlotta P, Menezes JRL and Macklis, JD (2007). Neuronal subtype specification in the cerebral cortex. Nature Reviews Neuroscience 8, 427-437

Monnier PP, Sierra A, Macchi P, Deitinghoff L, Andersen JS, Mann M, Flad M, Hornberger MR, Stahl B, Bonhoeffer F, Mueller BK. (2002) RGM is a repulsive guidance molecule for retinal axons. Nature. 419(6905), 392-5.

Moore SW, Correia JP, Lai Wing Sun K, Pool M, Fournier AE, Kennedy TE. (2008).Rho inhibition recruits DCC to the neuronal plasma membrane and enhances axon chemoattraction to netrin 1. Development. 135(17), 2855-64.

Muramatsu R, Nakahara S, Ichikawa J, Watanabe K, Matsuki N, Koyama R. (2010). The ratio of 'deleted in colorectal cancer' to 'uncoordinated-5A' netrin-1 receptors on the growth cone regulates mossy fibre directionality. Brain.133(Pt 1), 60-75

Nieto, M. et al. (2004) Expression of Cux-1 and Cux-2 in the subventricular zone and upper layers II-IV of the cerebral cortex. J. Comp. Neurol. 479, 168-180

Pascual M, Pozas E, Barallobre MJ, Tessier-Lavigne M, Soriano E. (2004).

Coordinated functions of Netrin-1 and Class 3 secreted Semaphorins in the guidance of reciprocal septohippocampal connections. Mol Cell Neurosci. 26(1), 24-33.

Paul LK, Brown WS, Adolphs R, Tyszka JM, Richards LJ, Mukherjee P and Sherr E $\mathrm{H}$ (2007). Agenesis of the corpus callosum: genetic, developmental and functional aspects of connectivity. Nature reviews Neuroscience 8, 287-299

Petit A, Sellers DL, Liebl DJ, Tessier-Lavigne M, Kennedy TE, Horner PJ. (2007). Adult spinal cord progenitor cells are repelled by netrin-1 in the embryonic and injured adult spinal cord. Proc Natl Acad Sci U S A. 104(45), 17837-42.

Picard M, Petrie RJ, Antoine-Bertrand J, Saint-Cyr-Proulx E, Villemure JF, Lamarche-Vane N. (2009) Spatial and temporal activation of the small GTPases RhoA and Rac1 by the netrin-1 receptor UNC5a during neurite outgrowth. Cell Signal. 21(12), 1961-73.

Powell AW, Sassa T, Wu Y, Tessier-Lavigne M, Polleux F. (2008). Topography of thalamic projections requires attractive and repulsive functions of Netrin-1 in the ventral telencephalon. PLoS Biol. 6(5), e116

Przyborski SA, Knowles BB, Ackerman SL. (1998). Embryonic phenotype of Unc5h3 mutant mice suggests chemorepulsion during the formation of the rostral cerebellar boundary. Development. 125(1), 41-50.

Rajagopalan S, Deitinghoff L, Davis D, Conrad S, Skutella T, Chedotal A, Mueller BK, Strittmatter SM. (2004) Neogenin mediates the action of repulsive guidance molecule. Nat Cell Biol. 6(8), 756-62 
Rakic P. (1995). A small step for the cell-a giant leap for mankind: a hypothesis of neocortical expansion during evolution. Trends Neurosci. 18, 383-388

Rash BG and Richards LJ. (2001). A role for cingulated pioneering axons in the development of the corpus callosum. J. Comp. Neurol. 434, 147-157

Ren T, Zhang J, Plachez C, Mori S, Richards LJ. (2007) Diffusion tensor magnetic resonance imaging and tract-tracing analysis of Probst bundle structure in Netrin1and DCC-deficient mice. J Neurosci. 27(39), 10345-9.

Richards LJ, Koester SE, Tuttle R, O'Leary DD. (1997). Directed growth of early cortical axons is influenced by a chemoattractant released from an intermediate target. J Neurosci. 17(7), 2445-58.

Richards LJ, Plachez C, Ren T. (2004). Mechanisms regulating the development of the corpus callosum and its agenesis in mouse and human. Clin Genet 66, 276-289

Rosenfeld JA, Ballif BC, Lucas A, Spence EJ, Powell C, Aylsworth AS, Torchia BA, Shaffer LG. (2009) Small deletions of SATB2 cause some of the clinical features of the $2 \mathrm{q} 33.1$ microdeletion syndrome. PLoS One. 4(8), e6568.

Saito T. (2006). In vivo electroporation in the embryonic mouse central nervous system. Nat Protoc 1(3), 1552-8.

Satterwhite E, Sonoki T, Willis TG, Harder L, Nowak R, Arriola EL, Liu H, Price HP, Gesk S, Steinemann D, Schlegelberger B, Oscier DG, Siebert R, Tucker PW, Dyer MJ. (2001) The BCL11 gene family: involvement of BCL11A in lymphoid malignancies. Blood. 98(12), 3413-20.

Senawong T, Peterson VJ, Avram D, Shepherd DM, Frye RA, Minucci S, Leid M. J (2003) Involvement of the histone deacetylase SIRT1 in chicken ovalbumin upstream promoter transcription factor (COUP-TF)-interacting protein 2-mediated transcriptional repression. Biol Chem. 278(44), 43041-50

Serafini T, Colamarino A.S, Leonardo E.D, Wang H, Beddington R, Skarnes C. W, and Tessier-Lavigne M. (1996). Netrin-1 is required for commissural axon guidance in the developing vertebrate nervous system. Cell 87, 1001-1014.

Sheehan-Rooney K, Pálinkášová B, Eberhart JK, Dixon MJ. (2010). A cross-species analysis of Satb2 expression suggests deep conservation across vertebrate lineages. Dev Dyn. 239(12), 3481-91

Shen, W. B., Plachez, C., Mongi, A. S. and Richards, L. J. (2006). Identification of candidate genes at the corticoseptal boundary during development. Gene Expr Patterns 6, 471-481

Shimiizu T and Hibi M. (2009). Formation and patterning of the forebrain and olfactory system by zinc-finger genes Fezf1 and Fezf2. Dev. Growth Differ. 51(3), 221-31 
Shoemaker LD, Orozco NM, Geschwind DH, Whitelegge JP, Faull KF, Kornblum HI. (2010). Identification of differentially expressed proteins in murine embryonic and postnatal cortical neural progenitors. PLoS One 5(2):e9121.

Shu T, Valentino KM, Seaman C, Cooper HM, Richards LJ. (2000). Expression of the netrin-1 receptor, deleted in colorectal cancer (DCC), is largely confined to projecting neurons in the developing forebrain. J Comp Neurol. 416(2), 201-12

Stancik, EK., Navarro-Quiroga, I., Sellke, R. and Haydar, T. F. (2010). Heterogeneity in ventricular zone neural precursors contributes to neuronal fate diversity in the postnatal neocortex. The Journal of Neuroscience 30(20), 7028-7036

Sugitani Y, Nakai S, Minowa O, Nishi M, Jishage K, Kawano H, Mori K, Ogawa M, Noda T. (2002). Brn-1 and Brn-2 share crucial roles in the production and positioning of mouse neocortical neurons. Genes Dev. 16(14), 1760-5.

Szemes M, Gyorgy A, Paweletz C, Dobi A, Agoston DV. (2006). Isolation and characterization of SATB2, a novel AT-rich DNA binding protein expressed in development- and cell-specific manner in the rat brain. Neurochem Res. 31(2), 23746.

Tarabykin, V., Stoykova, A., Usman, N. and Gruss, P. (2001). Cortical upper layer neurons derive from the subventricular zone as indicated by Svet1 gene expression.

Tcherkezian J, Brittis PA, Thomas F, Roux PP, Flanagan JG. (2010). Transmembrane receptor DCC associates with protein synthesis machinery and regulates translation. Cell. 141(4), 632-44

Tegay DH, Chan KK, Leung L, Wang C, Burkett S, Stone G, Stanyon R, Toriello HV, Hatchwell E. (2009). Toriello-Carey syndrome in a patient with a de novo balanced translocation $[46, \mathrm{XY}, \mathrm{t}(2 ; 14)(\mathrm{q} 33 ; \mathrm{q} 22)]$ interrupting SATB2. Clin Genet. 75(3), 259-64

Tomasch, J. (1954). Size, distribution, and number of fibers in the human corpus callosum. The Anat. Rec. 119, 119-135

Topark-Ngarm A, Golonzhka O, Peterson VJ, Barrett B Jr, Martinez B, Crofoot K, Filtz TM, Leid M. (2006). CTIP2 associates with the NuRD complex on the promoter of p57KIP2, a newly identified CTIP2 target gene. J Biol Chem. 281(43), 32272-83

Trichas G, Begbie J, Srinivas S. (2008). Use of the viral 2A peptide for bicistronic expression in transgenic mice. BMC Biol. 2008 Sep 15;6:40.

Urquhart J, Black GC, Clayton-Smith J. (2009). 1.4.5 Mb microdeletion in chromosome band 2q33.1 associated with learning disability and cleft palate. Eur J Med Genes 52(6), 454-7. 
Voelker CC, Garin N, Taylor JS, Gähwiler BH, Hornung JP, Molnár Z. (2004) Selective neurofilament (SMI-32, FNP-7 and N200) expression in subpopulations of layer V pyramidal neurons in vivo and in vitro. Cereb Cortex. 14(11), 1276-86

Wakabayashi Y, Watanabe H, Inoue J, Takeda N, Sakata J, Mishima Y, Hitomi J, Yamamoto T, Utsuyama M, Niwa O, Aizawa S, Kominami R. (2003). Bcl11b is required for differentiation and survival of $\alpha \beta T$ lymphocytes. Nature Immunology 4, 533-539

Watanabe K, Tamamaki N, Furuta T, Ackerman SL, Ikenaka K, Ono K. (2006) Dorsally derived netrin 1 provides an inhibitory cue and elaborates the 'waiting period' for primary sensory axons in the developing spinal cord Development. 133(7), $1379-87$

Xie Y, Hong Y, Ma XY, Ren XR, Ackerman S, Mei L, Xiong WC. DCC-dependent phospholipase $\mathrm{C}$ signaling in netrin-1-induced neurite elongation (2006) J Biol Chem. 281(5), 2605-11.

Xu B, Goldman JS, Rymar VV, Forget C, Lo PS, Bull SJ, Vereker E, Barker PA, Trudeau LE, Sadikot AF, Kennedy TE. (2010) Critical roles for the netrin receptor deleted in colorectal cancer in dopaminergic neuronal precursor migration, axon guidance, and axon arborization. Neuroscience. 169(2), 932-49.

Yamakawa K, Huot YK, Haendelt MA, Hubert R, Chen XN, Lyons GE, Korenberg JR. (1998) DSCAM: a novel member of the immunoglobulin superfamily maps in a Down syndrome region and is involved in the development of the nervous system. Hum Mol Genet. (2):227-37.

Yang Z, Liu N and Lin S. (2001). A zebrafish forebrain-specific zinc finger gene can induce ectopic dlx2 and dlx6 expression. Dev. Biol. 231(1), 138-48.

Zhu XJ, Wang CZ, Dai PG, Xie Y, Song NN, Liu Y, Du QS, Mei L, Ding YQ, Xiong WC. (2007) Myosin X regulates netrin receptors and functions in axonal path-finding. Nat Cell Biol. 9(2), 184-92.

Zimmer, C., Tiveron, M. C., Bodmer, R. and Cremer, H. (2004). Dynamics of Cux-2 expression suggests that an early pool of SVZ precursors is fated to become upper cortical layer neurons. Cereb. Cortex 14, 1408-1420 


\section{ACKNOWLEDGEMENTS}

I thank all members of Tarabykin lab (former and current) that helped and/or contributed to this work in anyway.

I would like to especially thank my good friends Dr. med. Athanasios Tzovoilis and Dr. med. Aggeliki Pantazi for support, help and encouragement.

I am also grateful for the unconditional support of all my family and friends back home, especially my father and my aunt Alexandra Kelesi.

My sincere gratefulness goes to my dear friend Dr. Charisios D. Tsiairis who has always been there for me, no matter what. His long-lived friendship, I cherish.

Special thanks goes to my lawyer Christian Tiedemann.

Last, but not least, my greatest gratitude extends to my mother (Freideriki KelesiSgourdou) who taught me independence, perseverance and dignity. She encouraged and supported, in every possible way, all endeavors I have ever made in my life. Without hesitation or second thought, she stood behind all my efforts and encouraged every single goal and dream I have ever had.

There is no other person to which I would rather dedicate this thesis, but my wonderful mother.

(Also, to all people in my life that hurt with my pain and rejoice with my happiness)

Science helps us understand the little we know, so we can know the lots we do not understand.

Paraskevi Sgourdou 
"Bevor dies erkannt werden konnte, bevor die innerste Abhängigkeit jeder Kunst von den Griechen, den Griechen von Homer bis auf Sokrates, überzeugend dargethan war, musste es uns mit diesen Griechen ergehen wie den Athenern mit Sokrates. Fast jede Zeit und Bildungsstufe hat einmal sich mit tiefem Missmuthe von den Griechen zu befreien gesucht, weil Angesichts derselben alles Selbstgeleistete, scheinbar völlig Originelle, und recht aufrichtig Bewunderte plötzlich Farbe und Leben zu verlieren schien und zur misslungenen Copie, ja zur Caricatur zusammenschrumpfte. Und so bricht immer von Neuem einmal der herzliche Ingrimm gegen jenes anmaassliche Völkchen hervor das sich erkühnte, alles Nichteinheimische für alle Zeiten als "barbarisch" zu bezeichnen: wer sind jene, fragt man sich, die, obschon sie nur einen ephemeren historischen Glanz, nur lächerlich engbegrenzte Institutionen, nur eine zweifelhafte Tüchtigkeit der Sitte aufzuweisen haben und sogar mit hässlichen Lastern gekennzeichnet sind, doch die Würde und Sonderstellung unter den Völkern in Anspruch nehmen, die dem Genius unter der Masse zukommt? Leider war man nicht so glücklich den Schierlingsbecher zu finden, mit dem ein solches Wesen einfach abgethan werden konnte: denn alles Gift, das Neid, Verläumdung und Ingrimm in sich erzeugten, reichte nicht hin, jene selbstgenugsame Herrlichkeit zu vernichten. Und so schämt und fürchtet man sich vor den Griechen; es sei denn, dass Einer die Wahrheit über alles achte und so sich auch diese Wahrheit einzugestehn wage, dass die Griechen unsere und jegliche Cultur als Wagenlenker in den Händen haben, dass aber fase immer Wagen und Pferde von zu geringem Stoffe und der Glorie ihrer Führer unangemessen sind, die dann es für einen Scherz erachten, ein solches Gespann in den Abgrund zu jagen: über den sie selbst, mit dem Sprunge des Achilles, hinwegsetzen."

Die Geburt der Tragödie, Friedrich Wilhelm Nietzsche (1872) 


\section{Curriculum Vitae}

Name: Paraskevi Sgourdou

Date of birth: November $28^{\text {th }} 1977$

Place of birth: Athens, Greece

Address (current): Gartenstrasse 1, apt. 20 37073, Goettingen

Address (permanent): 30 George Papandreou street

Zographou, 157-73, Athens, GREECE

E-mail address : psgourdou@gmail.com

\section{Education}

April 2008-current: $\mathrm{PhD}$ student at the university of Goettingen, in Goettingen Graduate School for Neurosciences and molecular Biosciences (GGNB) program (Germany)

September 2004-2007: graduate student at the University of Massachusetts, Amherst, Department of Biology, program in molecular and cellular biology (USA)

2002-2003: Research assistant/grad student, Hellenic National Research Foundation (Athens, Greece)

1996-2001: Student at the department of Biology, School of Applied Sciences, University of Patras (Greece)

1995: Graduated from the $28^{\text {th }}$ Lycium (high school) of Athens (Marasleio school).

\section{$\underline{\text { Foreign Languages }}$}

English: Proficiency (USA teaching assistantships)

French: Certificat de langue francaise (currently called DELFs) 


\section{$\underline{\text { Studies-research }}$}

Study of the localization of PSA lectin and the role of cell adhesion during the early stages of chick embryonic development (undergraduate diploma thesis)

$>$ Study/project and presentation of the impact that Bergson had on modern philosophy (course of philosophy of science, University of Patras)

$>$ Studying the MAPK pathway in colorectal cancer (Hellenic Research Foundation, 2002)

$>$ Completion of rotation projects at the University of Massachusetts, Amherst

Masters research project: Phenotypical and molecular characterization of the zebrafish 'accordion' motility mutant DA5.

PhD thesis project: The role of Satb2-Ctip2 and Fezl in cortical connectivity and the elucidation of their downstream pathway(s).

\section{Attendance/presentations in Congresses}

March 23-27, 2011: $9^{\text {th }}$ Goettingen Meeting of the German neuroscience society (Goettingen)

November 13-17, 2010: $40^{\text {th }}$ annual meeting of the Society For Neuroscience, San Diego, California (USA)

Poster presentaition entitled: The role of Satb2 and Ctip2 in cortical connectivity and the elucidation of their downstream pathways

September 29, 2010: Meeting of the German Anatomical Society, Wuerzburg (Germany)

Poster presentaition entitled: The role of Satb2 and Ctip2 in cortical connectivity and the elucidation of their downstream pathways

June 10-12, 2010: Berlin Neuroscience Forum, Liebenwalde, Germany Poster presentation entitled: The role of Satb2/Fezl and Ctip2 in cortical connectivity and the elucidation of their downstream pathways 
June 4-6, 2010: $5^{\text {th }}$ Interdisciplinary MPG PhDnet Meeting 2010, MPI for

Radioastronomy, Bonn, Germany

Poster presentation entitled: With or without corpus callosum

March 25-29, 2009: $8^{\text {th }}$ Goettingen Meeting of the German neuroscience society

Poster presentation entitled: The role of Satb2 and Ctip2 in cortical connectivity and the elucidation of their downstream pathways

May 22-25, 2008: International Cortical Development Meeting, Chania, Crete

June 14-18, 2006: $7^{\text {th }}$ International Conference on Zebrafish Development \&

Genetics, University of Wisconsin- Madison (USA)

Poster presentation entitled: Phenotypic analysis of the bajan mutant reveals defects in early locomotive behavior development.

Fall 2006: $8^{\text {th }}$ Annual Symposium, Center for Neuroendocrine studies (Umass, Amherst) poster presentation (USA)

$17^{\text {th }}$ Nov. 2005: Boston Area Graduate Student Symposium in Biomedical Sciences (Umass, medical school, USA)

Fall 2005: $7^{\text {th }}$ Annual Symposium, Center for Neuroendocrine studies (Umass, Amherst, USA)

7-9/3/2002: $3^{\text {rd }}$ Medical Chemistry Congress (Athens University, School for Sciences and Arts)

24-27/5/2001: $23^{\text {rd }}$ Panhellenic Congress of the Greek Society of Biological Sciences

27-29/10/2000: $15^{\text {th }}$ Annual Congress of the Greek Society of Neurosciences

25-28/5/2000: $22^{\text {rd }}$ Panhellenic Congress of the Greek Society of Biological Sciences

28-31/5/1999: $21^{\text {st }}$ Panhellenic Congress of the Greek Society of Biological Sciences

\section{Distinctions-Awards}

- $1^{\text {st }}$ award in interdepartmental literature competition (senior year of high school)

- $1^{\text {st }}$ award for essay on the philosophy of science $\left(3^{\text {rd }}\right.$ year of undergraduate studies, University of Patras, School of Applied Sciences, department of Biology, Greece)

- Second performance award in flute (forth year of musical studies) 


\section{$\underline{\text { Publications }}$}

Olson B., Sgourdou P. and Downes GB “Analysis Of A Zebrafish Behavioral Mutant Reveals A Dominant Mutation In atp2a1/SERCA1" (Genesis, 2010 Jun;48(6):354-61)

\section{$\underline{\text { Job Experience }}$}

$\checkmark$ Assistant in the pathologoanatomical laboratory of the Hareteio university hospital in Athens (summer 2000)

$\checkmark$ Assistant in the microbiological laboratory of the Hareteio university hospital in Athens (summer 2001)

$\checkmark$ Co-supervisor of the library of the department of Biology (program 3.20) at the University of Patras, Greece, 2000-2001

$\checkmark$ Teaching the lab course of Developmental Biology (senior year of undergraduate studies, University of Patras, Greece)

$\checkmark$ Tutoring prospective college students in the courses of Molecular and Cellular biology (Athens, Greece)

$\checkmark$ Teaching biochemistry/molecular biology and introductory biology laboratories (teaching assistantship) to freshmen and sophomores at the University of Massachusetts, Amherst, USA (2004-2007)

$\checkmark$ Teaching lab methods' courses and tutorials at the University of Goettingen (GGNB programme) 